\title{
ON MAXIMALLY ELLIPTIC SINGULARITIES
}

\author{
BY
}

\author{
STEPHEN SHING-TOUNG YAU
}

\begin{abstract}
Let $p$ be the unique singularity of a normal two-dimensional Stein space $V$. Let $m$ be the maximal ideal in $v_{p}$, the local ring of germs of holomorphic functions at $p$. We first define the maximal ideal cycle which serves to identify the maximal ideal. We give an "upper" estimate for maximal ideal cycle in terms of the canonical divisor which is computable via the topological information, i.e., the weighted dual graph of the singularity. Let $M \rightarrow V$ be a resolution of $V$. It is known that $h=\operatorname{dim} H^{1}(M, \theta)$ is independent of resolution. Rational singularities in the sense of $M$. Artin are equivalent to $h=0$. Minimally elliptic singularity in the sense of Laufer is equivalent to saying that $h=1$ and $\nu \theta_{p}$ is Gorenstein. In this paper we develop a theory for a general class of weakly elliptic singularities which satisfy a maximality condition. Maximally elliptic singularities may have $h$ arbitrarily large. Also minimally elliptic singlarities are maximally elliptic singularities. We prove that maximally elliptic singularities are Gorenstein singularities. We are able to identify the maximal ideal. Therefore, the important invariants of the singularities (such as multiplicity) are extracted from the topological information. For weakly elliptic singularities we introduce a new concept called "elliptic sequence". This elliptic sequence is defined purely topologically, i.e., it can be computed explicitly via the intersection matrix. We prove that $-K$, where $K$ is the canonical divisor, is equal to the summation of the elliptic sequence. Moreover, the analytic data $\operatorname{dim} H^{1}(M, \theta)$ is bounded by the topological data, the length of elliptic sequence. We also prove that $h=2$ and $v \theta_{p}$ Gorenstein implies that the singularity is weakly elliptic.
\end{abstract}

0. Introduction. Let $p$ be a singularity of a normal two-dimensional analytic space $V$. In [2], M. Artin introduced a definition for $p$ to be rational. Rational singularities have also been studied by, for instance, DuVal, Tyurina, Laufer and Lipman. In [37], Wagreich introduced a definition for $p$ to be weakly elliptic (see Definition 1.6). Weakly elliptic singularities have occurred naturally in papers by Grauert [7], Hirzebruch [12], Laufer [22], Orlik and Wagreich [27], [28] and Wagreich [37], [38]. Karras and Saito have studied some of these particular elliptic singularities. Recently, Laufer [24] developed a theory for a general class of weakly elliptic singularities which satisfy a minimality condition. These are the so-called minimally elliptic singularities. Suppose $V$ is a Stein space and $p$ is its only singularity. Let $\pi: M \rightarrow V$ be a resolution of $V$. It is known that $h=\operatorname{dim} H^{1}(M, \theta)$ is independent of

Received by the editors April 6, 1977.

AMS (MOS) subject classifications (1970). Primary 13C40, 13C45, 14B05, 14E15; Secondary $14 \mathrm{~J} 15,14 \mathrm{C} 20$.

Key words and phrases. Weakly elliptic singularity, elliptic sequence, maximally elliptic singularity, fundamental cycle, minimally elliptic cycle, Gorenstein singularity, canonical divisor, maximal ideal cycle, elliptic double points, Hilbert function, weighted dual graphs. 
resolution. One might classify singularities by $h$. Rational singularity is equivalent to $h=0$. Minimally elliptic singularity is equivalent to saying that $h=1$ and $v \theta_{p}$ is Gorenstein. In this paper, we develop a theory for a general class of weakly elliptic singularities which satisfy a maximality condition. Maximally elliptic singularities may have $h=\operatorname{dim} H^{1}(M, \theta)$ arbitrarily large. Also minimally elliptic singularities in the sense of Laufer [24] are maximally elliptic singularities. It is clear that maximally elliptic singularities should play an important role in the theory of normal two-dimensional singularities, especially from the point of view of classification of normal two-dimensional singularities. Our main results are the following.

THEOREM A. Let $\pi: M \rightarrow V$ be a resolution of a normal two-dimensional Stein space with $p$ as its only singular point. Suppose $\operatorname{dim} H^{1}(M, \theta) \geqslant 1$ and ${ }_{v} \hat{\theta}_{p}$ is Gorenstein; then the maximal ideal cycle cannot be greater than or equal to $-K^{\prime}$.

THEOREM B. Let $\pi: M \rightarrow V$ be a resolution of the normal two-dimensional Stein space with $p$ as its only singularity. Suppose ${ }_{v} \theta_{p}$ is Gorenstein and $H^{1}(M, \theta) \cong \mathbf{C}^{2}$. Then $p$ is a weakly elliptic singularity.

THeOREM C. Use the notation of Definition 3.3. Suppose $p$ is not a minimally elliptic singularity and $K^{\prime}$ exists. Then the elliptic sequence is of the following form:

$$
Z_{B_{0}}=Z, Z_{B_{1}}, \ldots, Z_{B_{l}}, Z_{B_{l+1}}=Z_{E}, \quad l \geqslant 0 .
$$

Moreover, $-K^{\prime}=\sum_{i=0}^{l} Z_{B_{i}}+E$ and $\operatorname{dim} H^{1}(M, \theta)<l+2=$ the length of the elliptic sequence.

TheORem D. Use the notation of Definition 3.3. Suppose $p$ is a maximally elliptic singularity. If $Z_{E} \cdot Z_{E} \leqslant-2$, then $m \theta=\theta(-Z)$, in particular, the multiplicity of ${ }_{V} \theta_{p}$ is equal to $-Z \cdot Z$. If $Z_{E} \cdot Z_{E} \leqslant-3$, then

$$
H^{0}(M, \vartheta(-Z)) \otimes_{\mathrm{C}} H^{0}(M, \vartheta(-n Z)) \rightarrow H^{0}(M, \vartheta(-(n+1) Z))
$$

is surjective for all $n>1$. If we assume further that the length of the elliptic sequence is equal to two, then the above map is surjective for all $n \geqslant 1$. In this case

$$
m^{n} \simeq H^{0}(A, \Theta(-n Z)) \text { for all } n \geqslant 1
$$

where $A=\pi^{-1}(p)$.

THEOREM E. If $p$ is a maximally elliptic singularity, then $p$ is a Gorenstein singularity.

One important question in the theory of normal two-dimensional singularities is "the identification of the maximal ideal $m$ of ${ }_{\nu} \theta_{p}$ ". In \$2.4 we first define the maximal ideal cycle which partially serves to identify the maximal ideal. In [2], the argument of M. Artin gave a lower estimate for maximal ideal cycle in terms of the fundamental cycle. Our Theorem A gives an "upper" estimate for maximal ideal cycle in terms of the canonical divisor 
which is computable via the topological information, i.e., the weighted dual graph. Theorem B permits us to develop a theory for those Gorenstein singularities with $\operatorname{dim} H^{1}(M, \theta)=2$. In $\$ 3.1$, we introduce a new concept called "elliptic sequence". It turns out that weakly elliptic singularities can be effectively studied by the method of elliptic sequence. For instance, it allows us to give a complete topological classification of elliptic double points. In order to compute the canonical divisor, one has to solve a system of linear equations, which is very painful. Theorem $\mathrm{C}$ provides us a quick and easy method to compute the canonical divisor. Moreover, it tells us that the analytic data $\operatorname{dim} H^{1}(M, \theta)$ is bounded by the topological data, the length of the elliptic sequence. We remark that the elliptic sequence is defined purely topologically, i.e., it can be computed easily via the intersection matrix. In Theorem $\mathrm{D}$, we are able to identify the maximal ideal. Therefore, the important invariants of the singularities (such as the multiplicity) are extracted from the topological information. Theorem $\mathrm{D}$ can also be used to compute the Hilbert function for the ring ${ }_{v} \Theta_{p}$. All complete intersection and hypersurface singularities are Gorenstein. Theorem E explains the reason why maximally elliptic singularities play an important role in the theory of normal two-dimensional singularities. We can also give a topological classification of maximally elliptic hypersurface singularities. However, the list is too long to be included.

We shall adopt Laufer's terminology [24] throughout this paper. Our presentation goes as follows:

In Chapter I we provide the necessary basic knowledge to read this paper. Most of it can be found in [24]. In Chapter II, we examine the structure of the exceptional set of weakly elliptic singularities and prove Laufer-type vanishing theorem.

We gratefully acknowledge the encouragement and help of Professor Henry B. Laufer during the investigation of these results. We would also like to thank Professors Bennett, Kuga, Sah, Siu and Wagreich for their encouragement and discussion of mathematics.

\section{Chapter I. Preliminaries}

Let $\pi: M \rightarrow V$ be a resolution of normal two-dimensional Stein space $V$. We assume that $p$ is the only singularity of $V$. Let

$$
\pi^{-1}(p)=A=\bigcup_{i} A_{i}, \quad 1 \leqslant i \leqslant n,
$$

be the decomposition of the exceptional set $A$ into irreducible components. Suppose $\pi$ is the minimal good resolution. The topological nature of the embedding of $A$ in $M$ is described by the weighted dual graph $\Gamma$ [14], [19]. The vertices of $\Gamma$ correspond to the $A_{i}$. The edge of $\Gamma$ connecting the vertices corresponding to $A_{i}$ and $A_{j}, i \neq j$, corresponds to the points of $A_{i} \cap A_{j}$. Finally, associated to each $A_{i}$ is its genus, $g_{i}$, as a Riemann surface, and its weight, $A_{i} \cdot A_{i}$, the topological self-intersection number. $\Gamma$ will denote the graph, along with the genera and the weights. 
Definition 1.1. $\operatorname{deg} A_{i}=\sum A_{j} \cdot A_{i}, j \neq i$.

A cycle (or divisorial cycle) $D$ on $A$ is an integral combination of the $A_{i}$. $D=\sum d_{i} A_{i}, 1 \leqslant i \leqslant n$, with $d_{i}$ an integer. In this paper, "cycle" will always mean a cycle on $A$. There is a natural partial ordering, denoted by $<$, between cycles defined by comparing the coefficients. We shall only be considering cycles $D \geqslant 0$. We let $\operatorname{supp} D=|D|=\cup A_{i}, d_{i} \neq 0$, denote the support of $D$.

Let $\theta$ be the sheaf of germs of holomorphic functions on $M$. Let $\theta(-D)$ be the sheaf of germs of holomorphic functions on $M$ which vanish to order $d_{i}$ on $A_{i}$. Let $\theta_{D}$ denote $\theta / \theta(-D)$. We use "dim" to denote dimension over $\mathbf{C}$.

$$
\chi(D)=\operatorname{dim} H^{0}\left(M, \theta_{D}\right)-\operatorname{dim} H^{1}\left(M, \theta_{D}\right) \text {. }
$$

Some authors work instead with the arithmetic genus $P_{a}(D)=1-\chi(D)$. The Riemann-Roch Theorem [31, p. 75] says

$$
\chi(D)=-\frac{1}{2}(D \cdot D+D \cdot K) \text {. }
$$

In (1.2), $K$ is the canonical divisor on $M . D \cdot K$ may be defined as follows. Let $\omega$ be a meromorphic 2-form on $M$, i.e. a meromorphic section of $K$. Let $(\omega)$ be the divisor of $\omega$. Then $D \cdot K=D \cdot(\omega)$ and this number is independent of the choice of $\omega$. In fact, let $g_{i}$ be the geometric genus of $A_{i}$, i.e. the genus of the desingularization of $A_{i}$. Then [31, p. 75]

$$
A_{i} \cdot K=-A_{i} \cdot A_{i}+2 g_{i}-2+2 \delta_{i}
$$

where $\delta_{i}$ is the "number" of nodes and cusps on $A_{i}$. Each singular point on $A_{i}$ other than a node or cusp counts as at least two nodes. Fortunately, such more complicated singularities will not occur in this paper.

The minimal resolution of $V$ is characterized by there being no $A_{i}$ which is a nonsingular rational curve with $A_{i} \cdot A_{i}=-1$ [7, p. 364]. The intersection matrix $\left(A_{i} \cdot A_{j}\right)$ is negative definite [26] so by (1.3) we see the following.

Proposition 1.2. $\pi$ is the minimal resolution of $V$ if and only if $A_{i} \cdot K>0$ for all $A_{i}$.

It follows immediately from (1.2) that if $B$ and $C$ are cycles then

$$
\chi(B+C)=\chi(B)+\chi(C)-B \cdot C .
$$

Associated to $\pi$ is a unique fundamental cycle $Z$ [2, pp. 131-132] such that $Z>0, A_{i} \cdot Z \leqslant 0$ all $A_{i}$, and such that $Z$ is minimal with respect to those two properties. $Z$ may be computed from the intersection matrix as follows [20, p. 607] via what is called a computation sequence (in the sense of Laufer) for $Z$ :

$$
\begin{aligned}
Z_{0}=0, Z_{1}=A_{i_{1}}, Z_{2} & =Z_{1}+A_{i_{2}}, \ldots, \\
Z_{j} & =Z_{j-1}+A_{i_{j}}, \ldots, Z_{l}=Z_{l-1}+A_{i_{l}}=Z,
\end{aligned}
$$

where $A_{i_{1}}$ is arbitrary and $A_{i_{j}} \cdot Z_{j-1}>0,1<j \leqslant l . \theta\left(-Z_{j-1}\right) / \theta\left(-Z_{j}\right)$ represents the sheaf of germs of sections of a line bundle over $A_{i_{j}}$ of Chern class $-A_{i_{j}} \cdot Z_{j-1}$. So 


$$
\begin{gathered}
H^{0}\left(M, \mathcal{O}\left(-Z_{j-1}\right) / \mathcal{O}\left(-Z_{j}\right)\right)=0 \text { for } j>1 . \\
0 \rightarrow \Theta\left(-Z_{j-1}\right) / \mathcal{O}\left(-Z_{j}\right) \rightarrow \Theta_{Z_{j}} \rightarrow \vartheta_{Z_{j-1}} \rightarrow 0
\end{gathered}
$$

is an exact sequence. From the long exact homology sequence for (1.5), it follows by induction that

$$
H^{0}\left(M, \theta_{Z_{k}}\right)=\mathbf{C}, \quad 1 \leqslant k \leqslant l,
$$

$$
\operatorname{dim} H^{1}\left(M, \theta_{Z_{k}}\right)=\sum \operatorname{dim} H^{1}\left(M, \vartheta\left(-Z_{j-1}\right) / \theta\left(-Z_{j}\right)\right),
$$

$$
1 \leqslant j \leqslant k \text {. }
$$

Since $M$ is two dimensional and not compact,

$$
H^{2}(M, \mathscr{F})=0
$$

for any coherent sheaf $\mathscr{F}$ on $M$ [33].

LEMMA 1.3. Let $Z_{k}$ be part of a computation sequence for $Z$ and such that $\chi\left(Z_{k}\right)=0$. Then $\operatorname{dim} H^{1}\left(M, \theta_{D}\right) \leqslant 1$ for all cycles $D$ such that $0 \leqslant D \leqslant Z_{k}$. Also, $\chi(D) \geqslant 0$.

Definition 1.4. A cycle $E>0$ is minimally elliptic if $\chi(E)=0$ and $\chi(D)>0$ for all cycles $D$ such that $0<D<E$.

Proposition 1.5. Let $Z_{k}>0$ be part of a computation sequence for the fundamental cycle and such that $\chi\left(Z_{k}\right)=0$. Let $B=\sum b_{i} A_{i}$ and $C=\sum c_{i} A_{i}$, $1 \leqslant i \leqslant n$, be any cycles such that $0<B, C \leqslant Z_{k}$ and $\chi(B)=\chi(C)=0$. Let $M=\Sigma \min \left(b_{i}, c_{i}\right) A_{i}, 1 \leqslant i \leqslant n$. Then $M>0$ and $\chi(M)=0$. In particular, there exists a unique minimally elliptic cycle $E$ with $E \leqslant Z_{k}$.

Wagreich [37] defined the singularity $p$ to be elliptic if $\chi(D)>0$ for all cycles $D>0$ and $\chi(F)=0$ for some cycles $F>0$. He proved that this definition is independent of the resolution. It is easy to see that under this hypothesis, $\chi(Z)=0$. The converse is also true [37], [24]. Henceforth, we will adopt the following definition.

DEFINITION 1.6. $p$ is said to be weakly elliptic if $\chi(Z)=0$.

The following analogue to Proposition 1.5 holds for weakly elliptic singularity.

Proposition 1.7. Suppose that $\chi(D) \geqslant 0$ for all cycles $D>0$. Let $B=$ $\sum b_{i} A_{i}$ and $C=\sum c_{i} A_{i}, 1 \leqslant i \leqslant n$, be any cycles such that $0<B, C$ and $\chi(B)=\chi(C)=0$. Let $M=\sum \min \left(b_{i}, c_{i}\right) A_{i}, \quad 1 \leqslant i \leqslant n$. Then $M>0$ and $\chi(M)=0$. In particular, there exists a unique minimally elliptic cycle $E$.

LEMMA 1.8. Let $E$ be a minimally elliptic cycle. Then for $A_{i} \subset \operatorname{supp} E$, $A_{i} \cdot E=-A_{i} \cdot K$. Suppose additionally that $\pi$ is the minimal resolution. Then $E$ is the fundamental cycle for the singularity having supp $E$ as its exceptional set. $A l s o$, if $E_{k}$ is part of a computation sequence for $E$ as a fundamental cycle and $A_{j} \subset \operatorname{supp}\left(E-E_{k}\right)$, then the computation sequence may be continued past $E_{k}$ so as to terminate at $E=E_{l}$ with $A_{i_{l}}=A_{j}$. 
THEOREM 1.9. Let $\pi: M \rightarrow V$ be the minimal solution of the normal twodimensional variety $V$ with one singular point $p$. Let $Z$ be the fundamental cycle on the exceptional set $A=\pi^{-1}(p)$. Then the following are equivalent:

(1) $Z$ is a minimally elliptic cycle,

(2) $A_{i} \cdot Z=-A_{i} \cdot K$ for all irreducible components $A_{i}$ in $A$,

(3) $\chi(Z)=0$ and any connected proper subvariety of $A$ is the exceptional set for a rational singularity.

Definition 1.10. Let $p$ be a normal two-dimensiona; singularity. $p$ is minimally elliptic if the minimal resolution $\pi: M \rightarrow V$ of a neighborhood of $p$ satisfies the conditions of Theorem 1.9.

\section{Chapter II. Basic Theory For Weakly Elliptic Singularities aNd MaXimal IDEAL CyCle}

1. Minimal good resolution of weakly elliptic singularities. In this section, we study the minimal good resolution of weakly elliptic singularities. We want to understand the nature of the computation sequence for the fundamental cycle $Z$ and what kind of curves can be in the exceptional fibre.

LEMMA 2.1. Let $\pi: M \rightarrow V$ be a resolution of the normal two-dimensional space $V$ with $p$ as its only singularity. Let $\pi^{-1}(p)=A=\cup A_{i}, 1<i<n$, be the decomposition of the exceptional set $A$ into irreducible components. Suppose there exists a minimally elliptic cycle $E$ on $A$. Then $\operatorname{supp} E=A_{1}$, if and only if either $A_{1}$ is a nonsingular elliptic curve or $A_{1}$ is a singular rational curve with node or cusp singularity. If $\operatorname{supp} E=\cup A_{i}, 1<i<k$, and $k>2$, then $\chi\left(A_{1}\right)=\cdots=\chi\left(A_{k}\right)=1$ and $A_{1}, \ldots, A_{k}$ are nonsingular rational curves.

Let $Z$ be the fundamental cycle on $A$. If $\chi(Z)=0$ and $n \geqslant 2$, then $\chi\left(A_{k+1}\right)$ $=\cdots=\chi\left(A_{n}\right)=1$. In particular, if supp $E$ consists of more than one irreducible component, then all $A_{i}, 1<i<n$, are nonsingular rational curves. If supp $E=A_{1}$, then all $A_{i}, 2 \leqslant i \leqslant n$, are nonsingular rational curves.

Proof. We claim that $\operatorname{supp} E=A_{1}$ if, and only if, $\chi\left(A_{1}\right)=0$. Suppose supp $E=A_{1}$. Then $E=n A_{1}$ for some positive integer $n$.

$$
\begin{aligned}
\chi\left(n A_{1}\right) & =\chi\left(A_{1}\right)+\chi\left((n-1) A_{1}\right)-(n-1) A_{1} \cdot A_{1} \\
& =n \chi\left(A_{1}\right)-\frac{1}{2} n(n-1) A_{1} \cdot A_{1} .
\end{aligned}
$$

Since $\chi(E)=0, \chi\left(A_{1}\right)=\frac{1}{2}(n-1) A_{1} \cdot A_{1}$. By definition of minimally elliptic cycle (Definition 1.10), $\chi\left(A_{1}\right)=\frac{1}{2}(n-1) A_{1} \cdot A_{1}>0$. However, $\chi\left(A_{1}\right)=$ $\frac{1}{2}(n-1) A_{1} \cdot A_{1}<0$. Therefore $\chi\left(A_{1}\right)=0$. Conversely, if $\chi\left(A_{1}\right)=0$, then $E=A_{1}$. This completes the proof of our claim. By (1.2) and (1.3),

$$
\begin{aligned}
\chi\left(A_{1}\right) & =-\frac{1}{2}\left(A_{1} \cdot A_{1}+A_{1} \cdot K\right), \quad \text { where } K \text { is the canonical } \\
& =-\frac{1}{2}\left(A_{1} \cdot A_{1}-A_{1} \cdot A_{1}+2 g_{1}-2+2 \delta_{1}\right), \quad \text { where } \delta_{1} \text { is the } \\
& =1-g_{1}-\delta_{1} .
\end{aligned}
$$


Therefore,

$$
\begin{array}{ll}
\chi\left(A_{1}\right)=0, & 1-g_{1}-\delta_{1}=0, \\
& \text { (1) } g_{1}=1 \text { and } \delta_{1}=0, \text { or } \\
& \text { (2) } g_{1}=0 \text { and } \delta_{1}=1 .
\end{array}
$$

So supp $E=A_{1}$ if either $A_{1}$ is a nonsingular elliptic curve or $A_{1}$ is a singular rational curve with node or cusp singularity. If $\operatorname{supp} E=A_{1} \cup \cdots \cup A_{k}$, $k>2$, then $\chi\left(A_{i}\right)>0$ for $A_{i} \subseteq \operatorname{supp} E$ by the definition of minimally elliptic cycle. On the other hand, $\chi\left(A_{i}\right) \leqslant 1$ by (1.1) and (1.6). So $\chi\left(A_{i}\right)=1$ and, hence, $1-g_{i}-\delta_{i}=1$ for $1 \leqslant i \leqslant k$. This implies that $g_{i}=0=\delta_{i}$, i.e. $A_{i}$, $1<i<k$, are nonsingular rational curves.

To prove the rest of the lemma, it suffices to show that if $\chi(Z)=0$, then $\chi\left(A_{i}\right)=1$ for $A_{i} \& \operatorname{supp} E . \chi(Z)=0$ implies that $\chi(D)>0$ for $D>0[24$, Corollary 4.3]. By (1.1) and (1.6), we know that $\chi\left(A_{i}\right) \leqslant 1$. So $0 \leqslant \chi\left(A_{i}\right) \leqslant 1$. However, $\chi\left(A_{i}\right)$ cannot be equal to zero by Proposition 1.7. Therefore $\chi\left(A_{i}\right)=1$ for $A_{i} \not \underline{\operatorname{supp}} E$, i.e., $A_{i} \underline{Z} \operatorname{supp} E$ are nonsingular rational curves.

Proposition 2.2. Let $\pi: M \rightarrow V$ be the minimal resolution for a weakly elliptic singularity p. Let $\pi^{\prime}: M^{\prime} \rightarrow V$ be the minimal resolution such that $A_{i}^{\prime}$ are nonsingular and have normal crossings, i.e. the $A_{i}^{\prime}$ meet transversely and no three meet at a point. Then $\pi=\pi^{\prime}$ and all the $A_{i}$ are rational curves except for the following cases.

(1) $A_{1}$ is a nonsingular elliptic curve. $A_{2}, \ldots, A_{n}$ are nonsingular rational curves. In this case, $\pi=\pi^{\prime}$. In fact, $0 \leqslant A_{i} \cdot A_{j} \leqslant 1$ for $i \neq j$.

(2) $A_{1}$ is a rational curve with a node singularity. $A_{2}, \ldots, A_{n}$ are nonsingular rational curves and have normal crossings. In fact, $0<A_{i} \cdot A_{j}<1$ for $i \neq j$.

(3) $A_{1}$ is a rational curve with a cusp singularity. $A_{2}, \ldots, A_{n}$ are nonsingular rational curves and have normal crossings. In fact $0<A_{i} \cdot A_{j}<1$ for $i \neq j$.

(4) All $A_{i}$ are nonsingular rational curves and have normal crossings except $A_{1}$ and $A_{2}$ having first order tangential contact at one point. In fact $A_{1} \cdot A_{2}=2$ and $0 \leqslant A_{i} \cdot A_{j} \leqslant 1$ for $i \neq j,(i, j) \neq(1,2)$ and $(i, j) \neq(2,1)$.

(5) All $A_{i}$ are nonsingular rational curves and have normal crossings except $A_{1}, A_{2}, A_{3}$ all meeting transversely at the same point. In fact, if $n>4$, then $0<A_{i} \cdot A_{j}<1$ for $1 \leqslant i \leqslant n, j \geqslant 4, i \neq j$ and $A_{1} \cdot A_{2}=1, A_{3} \cdot\left(A_{1}+A_{2}\right)=$ 2.

In case (2), $\pi^{\prime}$ has the following weighted dual graph as its subgraph:

$$
-w_{1} \bigodot_{-1} \text { with } w_{1} \geqslant 5 \text {. }
$$

In cases (3)-(5), $\pi^{\prime}$ has the following weighted dual graph as its subgraph:

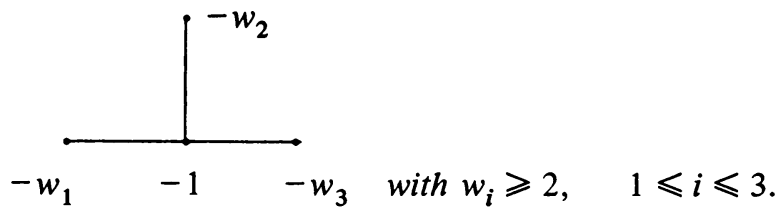


The proof is long but straightforward with many cases.

COROLlaRY 2.3. Let $\pi$ be the minimal resolution with nonsingular $A_{i}$ and normal crossings for a weakly elliptic singularity. Let $E$ be the minimally elliptic cycle, $E<Z$, the fundamental cycle. Then $E$ may be chosen as part of a computation sequence for $Z$ and $E=Z_{k}$. Moreover, if $Z_{j}<E$ is part of a computation sequence for $Z$ and $A_{m} \subset \operatorname{supp}\left(E-Z_{j}\right)$, then the computation sequence may be continued past $Z_{j}$ so that $A_{i_{k}}=A_{m}$.

PROof. The proof is the same as Corollary 3.6 of [24].

Proposition 2.4. Let $\pi$ be the minimal good resolution for a minimally elliptic singularity. Suppose $\pi$ is not the minimal resolution. Then the fundamental cycle is one of the following forms

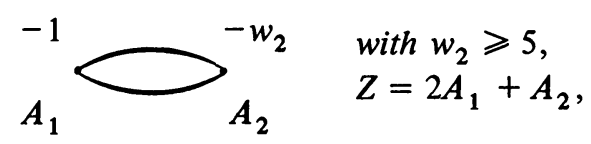

(II)

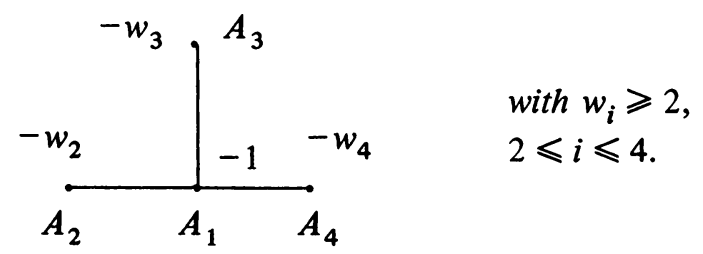

(1) If $A_{2} \cdot A_{2} \leqslant-3, A_{3} \cdot A_{3} \leqslant-3, A_{4} \cdot A_{4} \leqslant-3$, then

$$
Z=3 A_{1}+A_{2}+A_{3}+A_{4} \text {. }
$$

(2) If $A_{2} \cdot A_{2}=-2, A_{3} \cdot A_{3}=-3, A_{4} \cdot A_{4}<-6$, then

$$
Z=6 A_{1}+3 A_{2}+2 A_{3}+A_{4} \text {. }
$$

(3) If $A_{2} \cdot A_{2}=-2, A_{3} \cdot A_{3} \leqslant-4, A_{4} \cdot A_{4}<-4$, then

$$
Z=4 A_{1}+2 A_{2}+A_{3}+A_{4} \text {. }
$$

Proof. An easy case by case checking.

Proposition 2.5. Let $\pi: M \rightarrow V$ be the minimal good resolution of a normal two-dimensional Stein space with $p$ as its only weakly elliptic singular point.

Case 1. If supp $E$ has at least two irreducible components, then for any computation sequence of the form

$$
Z_{0}=0, Z_{1}=A_{i_{1}}, \ldots, Z_{k}=E, \ldots, Z_{l}=Z,
$$

we have $A_{i} \cdot Z_{j-1}=1$ for $j \neq k$ and $A_{i_{k}} \cdot Z_{k-1}=2$. If $\overline{\operatorname{supp} Z-\operatorname{supp} E} \neq \varnothing$, then for any $A_{i_{1}} \subseteq \overline{\operatorname{supp} Z-\operatorname{supp} E}$ we can choose a computation sequence of the form

$$
Z_{0}=0, Z_{1}=A_{i_{1}}, \ldots, Z_{r}, Z_{r+1}, \ldots, Z_{r+k}=E+Z_{r}, \ldots, Z_{l}=Z
$$

such that supp $Z_{r} \subseteq \overline{\operatorname{supp} Z-\operatorname{supp} E}$ and $Z_{r+1}-Z_{r}, \ldots, Z_{r+k}-Z_{r}=E$ is part of a computation sequence for $Z$. Moreover, any computation sequence of 
the above form has the following properties: $A_{i_{j}} \cdot Z_{j-1}=1$ for $j \neq r+k$ and $A_{i_{r+k}} \cdot Z_{r+k-1}=2$.

Case 2. If supp $E$ has only one irreducible component, then for any computation sequence of the form

$$
Z_{0}=0, Z_{1}=A_{i_{1}}=E, \ldots, Z_{l}=Z,
$$

we have $A_{i j} \cdot Z_{j-1}=1$ for all $j$. If $\overline{\operatorname{supp} Z-\operatorname{supp} E} \neq \varnothing$, then for any $A_{i} \subseteq$ supp $Z-\operatorname{supp} E$, we can choose a computation sequence of the form

$$
Z_{0}=0, Z_{1}=A_{i_{1}}, \ldots, Z_{r}, Z_{r+1}=Z_{r}+A_{i_{r+1}}, \ldots, Z_{l}=Z
$$

where $A_{i_{r+1}}=E$. Moreover, any computation sequence of the above form has $A_{i j} \cdot Z_{j-1}=1$ for all $j$.

Proof. Case 1.

$$
\begin{aligned}
0 & <A_{i_{k}} \cdot Z_{k-1}=A_{i_{k}} \cdot\left(E-A_{i_{k}}\right) \\
& =-A_{i_{k}} \cdot K-A_{i_{k}} \cdot A_{i_{k}} \quad \text { by Lemma } 1.8 \\
& =-2 g_{i_{k}}+2 .
\end{aligned}
$$

So $g_{i_{k}}=0$ and $A_{i_{k}} \cdot Z_{k-1}=2$. Since $\chi(Z)=0, H^{1}\left(M, \theta_{Z}\right)=\mathbf{C}$ by (1.1) and (1.6). As all $A_{i}$ are nonsingular rational curves, therefore (1.7) and the Riemann-Roch Theorem will show that $A_{i_{j}} \cdot Z_{j-1}=1$ for $j \neq k$.

From the above proof, we know that for any $A_{i} \subseteq$ supp $E$ such that there exist $A_{j} \underline{Z} \operatorname{supp} E$ and $A_{i} \cdot A_{j}>0$, then $e_{i}=1$ and $A_{i} \cdot A_{j}=1$, where $e_{i}$ is the coefficient of $A_{i}$ in $E$. It is easy to see that the computation sequence in Case 1 of the proposition can be chosen. Now we are going to prove the last statement of Case 1. By the above argument, we know $A_{i_{r+k}}\left(Z_{r+k-1}-Z_{r}\right)=$ 2 and, hence, $A_{i_{r+k}} \cdot Z_{r+k-1} \geqslant 2$ because $A_{i_{r+k}} \subseteq \operatorname{supp} E$ and $Z_{r} \subseteq \operatorname{supp~} Z-\operatorname{supp} E$. Since $H^{1}\left(M, \theta_{Z}\right)=C$, by (1.7) and the RiemannRoch Theorem, there is at most one $A_{i_{j}} \cdot Z_{j-1}=2$. So $A_{i_{r+k}} \cdot Z_{r+k-1}=2$ and $A_{i j} \cdot Z_{j-1}=1$ for $j \neq r+k$.

Case 2. Since $\chi(E)=\chi(Z)=0,(1.1)$ and (1.6) imply that $H^{1}\left(M, \theta_{Z}\right)=\mathbf{C}$ $=H^{1}\left(M, \Theta_{E}\right)$. So by $(1.7)$ and the Riemann-Roch Theorem, it follows immediately that $A_{i_{j}} \cdot Z_{j-1}=1$, for all $j$.

Now let us prove the last statement of Case 2. By Lemma 2.1 we know that $A_{i_{r+1}}$ is a nonsingular elliptic curve. Moreover, for any $A_{j} \neq A_{i_{r+1}}, A_{j}$ is a nonsingular rational curve. By $(1.7)$ and $H^{1}\left(M, \theta_{Z}\right)=\mathbf{C}$, we have

$$
\operatorname{dim} H^{1}\left(M, \vartheta\left(-Z_{r}\right) / \theta\left(-Z_{r}-A_{i_{r+1}}\right)\right)<1 .
$$

The Chern class of the line bundle associated to $\theta\left(-Z_{r}\right) / \theta\left(-Z_{r}-A_{i_{r+1}}\right)$ on $A_{i_{r+1}}$ is $-A_{i_{r+1}} \cdot Z_{r} \leqslant-1$. By the Serre duality theorem and the RiemannRoch Theorem,

$$
\operatorname{dim} H^{1}\left(M, \Theta\left(-Z_{r}\right) / \mathcal{O}\left(-Z_{r}-A_{i_{r+1}}\right)\right)=2 g_{i_{r+1}}-2+A_{i_{r+1}} \cdot Z_{r}=A_{i_{r+1}} \cdot Z_{r} .
$$

So

$$
A_{i_{r+1}} \cdot Z_{r}=1=\operatorname{dim} H^{1}\left(M, \vartheta\left(-Z_{r}\right) / \theta\left(-Z_{r}-A_{i_{r+1}}\right)\right)
$$


By (1.7), the Serre duality theorem and the Riemann-Roch Theorem, we know that $A_{i_{j}} \cdot Z_{j-1}=1$ for all $j$. Moreover, $A_{i_{j}}$ are nonsingular rational curves for $j \neq r+1$.

COROLlary 2.6. Let $\pi: M \rightarrow V$ be the minimal good resolution of a normal two-dimensional Stein space $V$ with $p$ as its only weakly elliptic singular point. Suppose $\operatorname{supp} E=A_{1}$. Let $Z=\sum z_{i} A_{i}$. Then $z_{1}=1$.

Proof. This is contained in the proof of Case 2 of the above proposition.

\section{Laufer-type vanishing theorem.}

Proposition 2.7. Let $p$ be a weakly elliptic singularity. Let $\pi: M \rightarrow V$ be the minimal good resolution of a Stein neighborhood $V$ of $p$ having $p$ as its only singular point. Let $Y>0$ be a cycle on the exceptional set $A$ such that $A_{i} \cdot Y \leqslant 0$ for all irreducible components $A_{i}$ of $A$. Let $Z$ be the fundamental cycle and $E$ the minimally elliptic cycle. Let $0=Z_{0}, \ldots, Z_{l}=Z$ be a computation sequence for $Z$ with $E=Z_{k}$ and $A_{i_{k}}$ such that $A_{i_{k}} \cdot Y<0$. Then $H^{1}\left(M, \vartheta\left(-Y-Z_{j}\right)\right)=0$ for $0 \leqslant j \leqslant l$.

Proof. The proof is similar to the proof of Lemma 3.11 in [24].

Proposition 2.8. Let $p, \pi, M, V, Y, Z$ and $E$ be as in Proposition 2.7. Let $E=\sum_{i=1}^{t} e_{i} A_{i}$. Suppose $E \cdot Y<0$. Let $A_{1}$ be an arbitrary $A_{i} \subset \operatorname{supp} E$. Then

$$
\rho: H^{0}(M, \vartheta(-Y)) \rightarrow H^{0}\left(M, \vartheta(-Y) / \vartheta\left(-Y-A_{1}\right)\right)
$$

is surjective if $A_{1}$ is an elliptic curve or if there exists $A_{j} \subseteq \operatorname{supp} E, A_{j} \neq A_{1}$ with $A_{j} \cdot Y<0$ or if $e_{1}>1$. If $A_{1}$ is a rational curve, $A_{j} \cdot Y=0$ for $A_{j} \neq A_{1}$, $A_{j} \subseteq \operatorname{supp} E$, and $e_{1}=1$, then the image of $\rho$ is a subspace $S$ of codimension 1 in $H^{0}\left(M, \vartheta(-Y) / \mathcal{O}\left(-Y-A_{1}\right)\right)$. If $\operatorname{dim} S \geqslant 2$, then the elements of $S$ have no common zeros as sections of line bundle $L$ on $A_{1}$ associated to $\theta(-Y) / \theta\left(-Y-A_{1}\right)$. If $\operatorname{dim} S=1$, then there is one common zero at a point $q \in A$ with $q \notin A_{j}$ where $A_{j} \cdot Y=0$ and $A_{j} \subseteq \operatorname{supp} E$.

Proof. The proof is similar to the proof of Lemma 3.12 in [24].

3. Structure theorem for weighted dual graphs of weakly elliptic singularities. For weighted dual graphs of weakly elliptic singularities, we can obtain some information from the following two propositions. Much more complete information is given in Chapter III.

Proposition 2.9. Let $\pi: M \rightarrow V$ be a resolution of a normal 2-dimensional Stein space $V$ with $p$ as its only weakly elliptic singularity. Let $E$ be the minimally elliptic cycle on $A=\pi^{-1}(p)$. Suppose $B$ is a connected subvariety of $A$ such that $B \succeq \operatorname{supp} E$. Then $B$ is the exceptional set of a rational singularity.

Proof. The fact that $B$ is exceptional in $M$ follows from [19, Lemma 5.11, p. 89]. Let $Z_{B}$ denote the fundamental cycle on $B$. It follows by [2, Theorem 3 , p. 132] that $\chi\left(Z_{B}\right) \leqslant 1$. On the other hand, since $p$ is a weakly elliptic singular point, $\chi\left(Z_{B}\right) \geqslant 0, \chi\left(Z_{B}\right)$ cannot be equal to zero. Otherwise it will 
contradict the minimality of the minimally elliptic cycle by Proposition 1.7 since $B \nsupseteq \operatorname{supp} E$. Therefore $\chi\left(Z_{B}\right)=1$. Applying Theorem 3 of [2], our result follows.

Proposition 2.10. Let $\pi: M \rightarrow V$ be a resolution of a normal twodimensional Stein space with $p$ as its only weakly elliptic singular point. Let $E$ be the minimally elliptic cycle on the exceptional set $A=\pi^{-1}(p)$. Suppose $B$ is a connected subvariety of $A$ containing $|E|$. Then $B$ is the exceptional set for weakly elliptic singularity. In particular, if $B=\operatorname{supp} E$, then $B$ is a minimally elliptic singularity.

Proof. As in Proposition 2.10, we know that $B$ is exceptional in $M$. Let $Z_{B}$ be the fundamental cycle on $B$. Then $\chi\left(Z_{B}\right) \leqslant 1$ by Theorem 3 of [2]. Since $p$ is a weakly elliptic singularity, so $\chi\left(Z_{B}\right) \geqslant 0$. Hence, $0 \leqslant \chi\left(Z_{B}\right) \leqslant 1 . \chi\left(Z_{B}\right)$ cannot be equal to one. Otherwise it will imply that $B$ is an exceptional set of rational singularity by Theorem 3 of [2]. Since $B \supseteq|E|$, Theorem 1 of [2] says that $\chi(E) \geqslant 1$. This is a contradiction, so $\chi\left(Z_{B}\right)=0$ and $B$ is the exceptional set for a weakly elliptic singularity.

4. Maximal ideal cycle. Let $\pi: M \rightarrow V$ be the resolution of a normal two-dimensional space $V$ with $p$ as its only singularity. Let $m$ be the maximal ideal in ${ }_{v} \theta_{p}$. One important question in normal two-dimensional singularity is the "identification of $m$ ". In this setion, we define the maximal ideal cycle which serves partially to identify the maximal ideal.

Definition 2.11. Let $A$ be the exceptional set in the resolution $\pi: M \rightarrow V$ of a 2-dimensional space $V$ with $p$ as its only singularity. Suppose that $\left\{A_{i}\right\}_{1<i<n}$ are the irreducible components of $A$. Let $m$ be the maximal ideal in ${ }_{v} \mathcal{O}_{p}$. If $f \in m$, then the divisor of $f,(f)=[f]+D$, where $[f]=\Sigma n_{i} A_{i}$ and $D$ does not involve any of $A_{i}$. Let $Y$ be the positive cycle such that $Y=$ $\inf _{f \in m}[f]$. Then $Y$ is called the maximal ideal cycle.

Proposition 2.12. Use the notation of Definition 2.11. The maximal ideal cycle is a positive cycle s.t. $Y \cdot A_{i} \leqslant 0$ for all $A_{i} \subseteq A$. In particular, $Y \geqslant Z$. In fact if $f_{1}, \ldots, f_{r} \in m$ such that $f_{1}, \ldots, f_{r}$ generate $m$, then $Y=\inf _{1<i<r}\left[f_{i}\right]$.

Proof. Easy.

Proposition 2.13. Use the notation of Definition 2.11. Let $Y$ be the maximal ideal cycle, then $m \theta \subseteq \theta(-Y)$. Moreover, if $m \theta$ is locally principal, i.e. $m \theta=\theta(-D)$ for some positive divisor $D$, then $D=Y$ and $m \theta=\theta(-Y)$.

Proof. Easy.

DEFINITION 2.14. Let $\sigma: M^{\prime} \rightarrow M$ be a monoidal transformation with center $q \in M$. We associate with the curve $C \subseteq M, q \notin C$, the curve $C^{*}$, the proper transform of $C$ in $M^{\prime}$. If $q$ is a point of multiplicity $n$ of the curve $C$, we associate with this curve the curve $C^{*}+n L \subset M^{\prime}$ where $L=\sigma^{-1}(q)$. With the divisor $Z=\Sigma k_{i} C_{i}$, we associate the divisor $\sigma^{*}(Z)=\Sigma k_{i} C_{i}^{*}+k_{i} n_{i} L$, where $n_{i}$ is the multiplicity of the point $q$ on the curve $C_{i}$. 
LEMMA 2.15. Let $\pi: M \rightarrow V$ be a resolution of a normal two-dimensional analytic space with $p$ as its only singularity. Let $A=\pi^{-1}(p)=\cup A_{i}$ be the decomposition of $A$ into irreducible components. Suppose $W$ is a positive cycle on $A$ such that $W \cdot A_{j} \leqslant 0$ for all $A_{j} \subseteq A$. For any positive cycle $X$ on $A$ such that $X>W, X^{2} \leqslant W^{2}$. Also, $X^{2}=W^{2}$ if and only if $X=W$.

Proof. Let $X=W+\sum n_{i} A_{i}$ where $n_{i} \geqslant 0$. Then

$$
X^{2}=W^{2}+2 \sum n_{i}\left(A_{i} \cdot W\right)+\sum_{i, j} n_{i} n_{j}\left(A_{i} \cdot A_{j}\right)
$$

Now $A_{i} \cdot W \leqslant 0$ by the hypothesis. The last expression is nonpositive since $\left(A_{i} \cdot A_{j}\right)$ is negative definite. Moreover, this expression is zero if and only if $n_{i}=0$ for all $i$ by the definiteness.

LEMMA 2.16. Let $\pi: M \rightarrow V$ be a normal two-dimensional analytic space with $p$ as its only singularity. Let $A=\pi^{-1}(p)=\cup_{i=1}^{t} A_{i}$ be the decomposition of $A$ into irreducible components. Let $\sigma: M^{\prime} \rightarrow M$ be a monoidal transformation with point $q$ as center. Let $D=\pi^{-1}(q)$ and $A_{i}$ be the proper transform of $A_{i}^{\prime}$ by $\sigma$. Then

$$
(\pi \cdot \sigma)^{-1}(p)=D \cup\left(\bigcup_{i=1}^{t} A_{i}^{\prime}\right)
$$

Suppose $X$ is a positive cycle on $A$ such that $A_{i} \cdot X<0$ for all $A_{i} \subseteq A$. Then $D \cdot \sigma^{*}(X)=0$ and $A_{i}^{\prime} \cdot \sigma^{*}(X) \leqslant 0$ for all $1 \leqslant i \leqslant t$.

Proof. Since $A_{i}$ is linearly equivalent to some divisor not passing through $q$, hence $X$ is also linearly equivalent to some divisor not passing through $q$. It follows that $\pi^{*}(X) \cdot D=0$. By p. 421 of [37], $X \cdot A_{i}=\sigma^{*}(X) \cdot \sigma^{*}\left(A_{i}\right)$. So $0 \geqslant X \cdot A_{i}$ implies that

$$
0 \geqslant \sigma^{*}(X) \cdot \sigma^{*}\left(A_{i}\right)=\sigma^{*}(X)\left(A_{i}^{\prime}+m_{i} D\right)=\sigma^{*}(X) \cdot A_{i}^{\prime} .
$$

THEOREM 2.17. Let $\pi: M \rightarrow V$ be a normal two-dimensional analytic space with $p$ as its only singularity. Let $A=\pi^{-1}(p)=\cup_{i=1}^{t} A_{i}$ be the decomposition of $A$ into irreducible components. Let $Y$ be the maximal ideal cycle associated to $\pi$. Then the multiplicity of $v_{p} \geqslant-Y \cdot Y$. If $m \theta$ is locally principal, then the multiplicity of ${ }_{v} \theta_{p}=-Y \cdot Y$.

Proof. If $m \theta$ is locally principal, then $m \theta=\theta(-Y)$ by Proposition 2.13. In this case Theorem 2.7 of [37] says that multiplicity of. $v_{p}$ is equal to $-Y \cdot Y$.

In the general case, let $\pi^{\prime}: M^{\prime} \rightarrow M$ be the monoidal transformation with center $m \theta$. The map $\pi^{\prime}$ is a composition of monoidal transformations $\sigma$ with points as center (see [42, lemma, p. 538]). Let $A^{1}=\left(\pi \cdot \pi^{\prime}\right)^{-1}(p)=\cup_{i=1}^{s} A_{i}^{\prime}$. Then Lemma 2.16 says that $A_{i}^{\prime} \cdot \pi^{\prime *}(Y)<0$ for all $1<i<s$. Let $\theta^{\prime}$ be the structure sheaf on $M^{\prime}$. Let $Y^{\prime}$ be the maximal ideal cycle relative to $\pi \cdot \pi^{\prime}$. 
Then $m \theta^{\prime}=\theta^{\prime}\left(-Y^{\prime}\right)$. But $m \theta^{\prime} \subseteq \theta^{\prime}\left(-\pi^{\prime *}(Y)\right)$. So $Y^{\prime} \geqslant \pi^{\prime *}(Y)$. Theorem 2.7 of [37] and Lemma 2.15 will show that the multiplicity $\left({ }_{V} \theta_{p}\right)=-Y^{\prime} \cdot Y^{\prime}$ $>-\left[\pi^{*}(Y)\right]^{2}$. However, for any proper modification $\sigma$ and divisor $L$, we know that $\left[\sigma^{*}(L)\right]^{2}=L^{2}$. So $\left(\pi^{\prime *}(L)\right)^{2}=L^{2}$. In particular, $\left(\pi^{* *}(Y)\right)^{2}=Y^{2}$. Therefore multiplicity of ${ }_{\nu} \theta_{p} \geqslant-Y^{2}$.

DEFINITION 2.18. Let $p$ be the only singularity of the normal two-dimensional space $V$. Let $\pi: M \rightarrow V$ be the resolution of $V$. Let $A=\cup A_{i}$, $1 \leqslant i \leqslant n$, be the decomposition of $A=\pi^{-1}(p)$ into irreducible components. Let $K$ be the canonical divisor on $M$. We define the negative cycle $K^{\prime}=$ $\sum k_{i} A_{i}$ on $A$ where $k_{i} \in \mathbf{Z}$, the set of integers, to be a cycle such that $A_{i} \cdot K^{\prime}=A_{i} \cdot K$ for all $A_{i} \subseteq A$. ( $K^{\prime}$ does not always exist.)

The following theorem gives a "nonlower" estimate of the maximal ideal cycle in terms of the cycle $K^{\prime}$.

THEOREM 2.19. Let $\pi: M \rightarrow V$ be the minimal resolution of a normal two-dimensional Stein space with $p$ as its only singular point. Suppose $K^{\prime}$ exists and $\operatorname{dim} H^{1}(M, \theta) \geqslant 2$; then the maximal ideal cycle $Y$ relative to $\pi$ cannot be greater than or equal to $-K^{\prime}$.

Proof. By Theorem 3.2, p. 603 of [20], we know that $H^{1}\left(M, \theta\left(K^{\prime}\right)\right)=0$. The following cohomology exact sequence,

$$
H^{1}\left(M, \theta\left(K^{\prime}\right)\right) \rightarrow H^{1}(M, \theta) \rightarrow H^{1}\left(M, \theta_{-K^{\prime}}\right) \rightarrow 0,
$$

shows that $H^{1}\left(M, \Theta_{-K^{\prime}}\right) \cong H^{1}(M, \vartheta)$. Since

$$
\begin{aligned}
\chi\left(-K^{\prime}\right) & =-\frac{1}{2}\left[\left(-K^{\prime}\right) \cdot K+\left(-K^{\prime}\right) \cdot\left(-K^{\prime}\right)\right] \\
& =-\frac{1}{2}\left[\left(-K^{\prime}\right) \cdot K^{\prime}+\left(-K^{\prime}\right)\left(-K^{\prime}\right)\right]=0
\end{aligned}
$$

by (1.2), hence (1.1) says that

$$
\operatorname{dim} H^{0}\left(M, \Theta_{-K^{\prime}}\right)=\operatorname{dim} H^{1}\left(M, \theta_{-K^{\prime}}\right)=\operatorname{dim} H^{1}(M, \theta) \geqslant 2 .
$$

Suppose on the contrary that $Y \geqslant-K^{\prime}$. Since $\pi$ is the minimal resolution, $A_{i} \cdot K^{\prime} \geqslant 0$ for all $A_{i}$, so $-K^{\prime} \geqslant Z$ by the definition of the fundamental cycle $Z$. It follows that there is a natural injective map

$$
H^{0}\left(M, \vartheta\left(K^{\prime}\right)\right) \rightarrow H^{0}(M, \vartheta(-Z)) \text {. }
$$

We claim that this map is actually surjective. Given any $g \in H^{0}(M, \theta(-Z))$, we know that $g$ is actually a function on $V$ which vanishes at $p$. By Proposition 2.13, $g \in H^{0}(M, \theta(-Y))$. However, $Y>-K^{\prime}$ implies that $H^{0}(M, \theta(-Y)) \subseteq H^{0}\left(M, \theta\left(K^{\prime}\right)\right)$. So $g$ can also be considered as an element in $H^{0}\left(M, \Theta\left(K^{\prime}\right)\right)$. This proves our claim. Look at the following commutative diagram with exact rows.

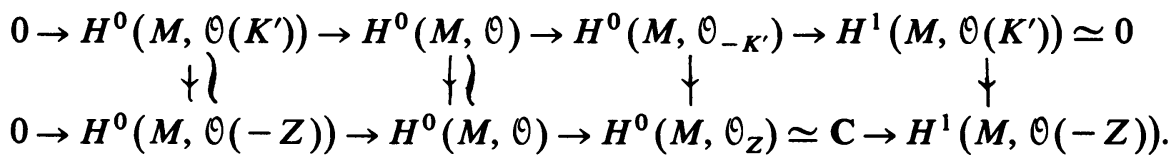

Since $H^{0}\left(M, \theta_{Z}\right) \simeq \mathbf{C}$ by (1.6), so $H^{0}(M, \vartheta) \rightarrow H^{0}\left(M, \theta_{Z}\right)$ is surjective. 
We have $H^{0}\left(M, \theta_{-}\right)$is isomorphic to $H^{0}\left(M, \theta_{Z}\right)$. However, $\operatorname{dim} H^{0}\left(M, \theta_{Z}\right)=1<\operatorname{dim} H^{0}\left(M, \theta_{-K^{\prime}}\right)$. This leads to a contradiction. Q.E.D.

If ${ }_{V} \theta_{p}$ is a Gorenstein ring, i.e. there is some neighborhood $Q$ of $p$ in $V$ and a holomorphic 2 -form $\omega$ on $Q-p$ such that $\omega$ has no zeros on $Q-p$, then $K^{\prime}$ exists.

THEOREM 2.20. If we assume ${ }_{\nu} \theta_{p}$ is Gorenstein in Theorem 2.19, then the same result holds even if $\pi$ is not necessarily the minimal resolution.

Proof. As ${ }_{V} \theta_{p}$ is Gorenstein, there exists $\omega \in H^{0}(M-A, \Omega)$ having no zeros near $A$. Serre duality gives $H^{1}(M, \theta)$ as dual to $H_{*}^{1}(M, \Omega)$, where $\Omega$ is the canonical sheaf, i.e. the sheaf of germs of holomorphic 2-forms. By Theorem 3.4, p. 604 of [20], for suitable $M$, which can be chosen to be arbitrarily small neighborhoods of $A=\pi^{-1}(p), H_{*}^{1}(M, \Omega)$ may be identified with $H^{0}(M-A, \Omega) / H^{0}(M, \Omega)$. So

$$
\operatorname{dim} H^{0}(M-A, \Omega) / H^{0}(M, \Omega)=n \geqslant 2 .
$$

There exist $\omega_{2}, \ldots, \omega_{n}$ in $H^{0}(M-A, \Omega)$ such that the image of $\omega, \omega_{2}, \ldots, \omega_{n}$ in $H^{0}(M-A, \Omega) / H^{0}(M, \Omega)$ forms a basis. Since $\omega$ is nonzero in a neighborhood of $A$, we may assume that $\omega_{i}=f_{i} \omega, 2 \leqslant i<n$, where $f_{i} \in H^{0}(M, \theta)$. Moreover we can assume that $f_{i}$ are vanishing at $p$, i.e., $f_{i} \in H^{0}(M, m \vartheta)$. Otherwise we simply replace $f_{i}$ by $f_{i}-f_{i}(p), 2<i<n$.

Suppose our theorem is false. Then the maximal ideal cycle $Y>[\omega]$. Since $m \theta \subseteq \theta(-Y)$ by Proposition 2.13, we have $\omega_{i}=f_{i} \omega, 2<i<n$, all in $H^{0}(M, \Omega)$. This contradicts the fact that the image of $\omega, \omega_{2}, \ldots, \omega_{n}$ forms a basis for $H^{0}(M-A, \Omega) / H^{0}(M, \Omega)$.

\section{Chapter III. Elliptic SeQuences AND \\ Maximally Elliptic Singularities}

One might classify hypersurface singularities by $h=\operatorname{dim} H^{1}(M, \theta)$. If $h=0$, then the singularity is rational [20]. If $h=1$, then the singularity is minimally elliptic [24]. Let us consider the condition $h=2$. All hypersurface singularities, as well as complete intersection, are Gorenstein, so the following theorem applies.

THEOREM 3.1. Let $\pi: M \rightarrow V$ be a resolution of the normal two-dimensional Stein space $V$ with $p$ as its only singularity. Suppose $v_{p}$ is Gorenstein and $H^{1}(M, \theta)=C^{2}$. Then $p$ is a weakly elliptic singularity.

Proof. Let $\pi^{-1}(p)=A=\cup A_{i}, 1 \leqslant i \leqslant n$, be the decomposition of the exceptional set $A$ into irreducible components and $Z$ be the fundamental cycle on $A$. Since $H^{1}(M, \theta)$ is independent of the choice of the resolution [20, Lemma 3.1, p. 599], [2, p. 124], we may assume that $\pi$ is the minimal good resolution. By $(1.6), H^{0}\left(M, \theta_{z}\right)=$ C. So we have the following exact cohomology sequence:

$$
0 \rightarrow H^{1}(M, \vartheta(-Z)) \rightarrow H^{1}(M, \theta) \rightarrow H^{1}\left(M, \theta_{Z}\right) \rightarrow 0 .
$$


Since $H^{1}(M, \theta)=C^{2}, \operatorname{dim} H^{1}\left(M, \theta_{Z}\right)$ is either 0,1 or 2. If $H^{1}\left(M, \theta_{Z}\right)=0$, then $\chi(Z)=\operatorname{dim} H^{0}\left(M, \theta_{Z}\right)-\operatorname{dim} H^{1}\left(M, \theta_{Z}\right)=1$, i.e. $p(Z)=1-\chi(Z)$ $=0$. By Theorem 3 of [2], $p$ is a rational singularity. However, as $H^{1}(M, \theta)$ $=\mathbf{C}^{2}$, the first direct image $R^{1} \pi^{*} \theta_{V}$ is not zero by Lemma 3.1 of [20]. This leads to a contradiction. If $H^{1}\left(M, \theta_{Z}\right)=C^{2}$, then $H^{1}(M, \theta(-Z))=0$. As $v^{\vartheta_{p}}$ is Gorenstein, there exists $\omega \in H^{0}(M-A, \Omega)$ having no zeros near $A$, where $\Omega$ is the canonical sheaf, i.e. the sheaf of germs of holomorphic 2-forms. By Theorem 3.4, p. 604 of [20], for suitable $M$, which can be chosen to be arbitrarily small neighborhoods of $A=\pi^{-1}(p), H_{*}^{1}(M, \Omega)$ may be identified with $H^{0}(M-A, \Omega) / H^{0}(M, \Omega)$. So $\operatorname{dim} H^{0}(M-A, \Omega) / H^{0}(M, \Omega)$ $=2$ and there exists $\omega^{\prime} \in H^{0}(M-A, \Omega)$ such that the image of $\omega$, $\omega^{\prime}$ in $H^{0}(M-A, \Omega) / H^{0}(M, \Omega)$ forms a basis. Since $\omega$ is nonzero in a neighborhood of $A$, we may assume that $\omega^{\prime}=f \omega$ where $f \in H^{0}(M, \theta)$. Furthermore, replacing $f$ by $f-f(p)$, if necessary, we can assume that $f \in$ $H^{0}(M, m \theta)$. Let $w_{i}$ be the order of the pole of $\omega$ on $A_{i}$. Consider a cover as in Lemma 3.8 of [24]. On $P_{1}$,

$$
\omega=\left(\omega_{1}\left(x_{1}, y_{1}\right) / y_{1}^{w_{1}}\right) d x_{1} \wedge d y_{1}
$$

where $\omega_{1}\left(x_{1}, y_{1}\right)$ is a holomorphic function, $\omega_{1}\left(x_{1}, 0\right) \not 0$. There is a holomorphic function $f\left(x_{1}\right), r \leqslant x_{1} \leqslant R$, such that

$$
\int_{\substack{\left|x_{1}\right|=R \\\left|y_{1}\right|=R}} y_{1}^{w_{1}-1} f\left(x_{1}\right) \frac{\omega_{1}\left(x_{1}, y_{1}\right)}{y_{1}^{w_{1}}} d x_{1} \wedge d y_{1} \neq 0 .
$$

Let $\lambda_{01}=y_{1}^{w_{1}-1} f\left(x_{1}\right)$ and $\lambda_{0 j}=0$ for $2 \leqslant j \leqslant t$. Then by Lemma 3.8 of [24], $\operatorname{cls}[\lambda] \neq 0$ in $H^{1}(M, \vartheta)$. Let $Z=\sum z_{i} A_{i}, 1 \leqslant i \leqslant n$, be the fundamental cycle. If $w_{1}-1 \geqslant z_{1}$, then $\lambda$ may be thought of as also a cocycle in $H^{1}(N(\mathcal{Q})$, $\theta(-Z)$ ). So cls $[\lambda]=0$ in $H^{1}\left(M^{\prime}, \theta(-Z)\right.$ ) and necessarily in $H^{1}\left(M^{\prime}, \theta\right)$. Thus $w_{1}-1>z_{1}$ is impossible, i.e. $w_{1} \leqslant z_{1}$. As $m \theta \subseteq \theta(-Z)$, p. 133 of [2], we have $\omega^{\prime}=f \omega \in H^{0}(M, \Omega)$, i.e., $\omega, \omega^{\prime}$ cannot form a basis for $H^{0}(M-$ $A, \Omega) / H^{0}(M, \Omega)$. This is a contradiction. So the only possible case is $H^{1}\left(M, \theta_{z}\right)=$ C. Hence $\chi(Z)=\operatorname{dim} H^{0}\left(M, \theta_{z}\right)-\operatorname{dim} H^{1}\left(M, \theta_{Z}\right)=0$, i.e. $p$ is a weakly elliptic singularity. Q.E.D.

However, that $\operatorname{dim} H^{1}(M, \theta)=3$ and ${ }_{\nu} \theta_{p}$ is Gorenstein do not imply $p$ is a weakly elliptic singularity.

ExAmple. Let $V$ be the locus in $C^{3}$ of $z^{2}=x^{6}+y^{6}$. Then the dual weighted graph is

$$
\begin{gathered}
g=2 \\
-1
\end{gathered}
$$

It can be calculated by [23] that $\operatorname{dim} H^{1}(M, \theta)=3$.

THEOREM 3.2. Let $V$ be a normal two-dimensional Stein space with $p$ as its only singularity. Suppose ${ }_{V} \theta_{p}$ is Gorenstein, i.e. there is some neighborhood $Q$ of $p$ in $V$ and a holomorphic 2-form $\omega$ on $Q-p$ such that $\omega$ has no zeros on $Q-p$. If there exists $f \in{ }_{v} \theta_{p}$ such that $\omega, f \omega, f^{2} \omega, \ldots, f^{n-1} \omega$ is a basis for $H_{*}^{1}(M, \Omega)$, then $p$ is a weakly elliptic singular point. 
Proof. Replacing $f$ by $f-f(p)$, if necessary, we may assume that $f \in$ $H^{0}(M, m \vartheta)$. By $(1.6), H^{0}\left(M, \theta_{Z}\right)=$ C. So we have the following exact cohomology sequence.

$$
0 \rightarrow H^{1}(M, \vartheta(-Z)) \rightarrow H^{1}(M, \theta) \rightarrow H^{1}\left(M, \theta_{Z}\right) \rightarrow 0 .
$$

By Theorem 3.1, we need only consider the case $n \geqslant 3$. It is easy to see that $\operatorname{dim} H^{1}\left(M, \theta_{z}\right)>0$. Otherwise, as observed in the proof of Theorem 3.1, $p$ will be a rational singular point. To prove that $p$ is a weakly elliptic singular point, it suffices to show that $H^{1}\left(M, \theta_{Z}\right)=\mathbf{C}$. Suppose on the contrary that $\operatorname{dim} H^{1}\left(M, \Theta_{Z}\right) \geqslant 2$. Then $\operatorname{dim} H^{1}(M, O(-Z)) \leqslant n-2$. Let the notation be as in the proof of Theorem 3.1. We know that there exists $A_{1} \subseteq A$ such that on $P_{1}$,

$$
f^{i} \omega=\frac{\omega_{1}\left(x_{1}, y_{1}\right)}{y_{1}^{-i a_{1}+w_{1}}} d x_{1} \wedge d y_{1}, \quad w_{1}-i a_{1}>0,0<i<n-1,
$$

where $\omega_{1}\left(x_{1}, y_{1}\right)$ is a holomorphic function, $\omega_{1}\left(x_{1}, 0\right) \not \equiv 0 .(\omega)=-\sum w_{i} A_{i}$ and $(f)=\Sigma a_{i} A_{i}+D=[f]+D$. $D$ does not involve any $A_{i}$. There are holomorphic functions $g_{i}\left(x_{1}\right), r \leqslant x_{1} \leqslant R$, such that

$$
\int_{\substack{\left|x_{1}\right|=R \\\left|y_{1}\right|=R}} y_{1}^{w_{1}-i a_{1}-1} g_{i}\left(x_{1}\right) \frac{\omega_{1}\left(x_{1}, y_{1}\right)}{y_{1}^{w_{1}-i a_{1}}} d x_{1} \wedge d y_{1} \neq 0 .
$$

Let $\lambda_{01}^{i}=y_{1}^{w_{1}-i a_{1}-1} g_{i}\left(x_{1}\right)$ and $\lambda_{0 j}^{i}=0$ for $2 \leqslant j \leqslant t$. Then by Lemma 3.8 of [24], $\operatorname{cls}\left[\lambda^{i}\right] \neq 0$ in $H^{1}(M, \theta)$. In fact, $\left\{\lambda^{i}\right\}$ forms a basis for $H^{1}(M, \theta)$ because $\left\langle\lambda^{i}, f^{j} \omega\right\rangle=0$ for $i \neq j$. Since

$$
\operatorname{dim} H^{1}(M, \mathcal{\theta})-\operatorname{dim} H^{1}(M, \mathcal{O}(-Z))=\operatorname{dim} H^{1}\left(M, \mathcal{O}_{Z}\right)>2,
$$

there are at least two $\lambda^{i_{1}}, \lambda^{i_{2}}$ which are not in $H^{1}(M, \mathcal{O}(-Z))$. Hence, $w_{1}-i_{1} a_{1}-1<z_{1}$ and $w_{1}-i_{2} a_{1}-1<z_{1}$, i.e., $w_{1} \leqslant z_{1}+i_{1} a_{1}, w_{1}<z_{1}+$ $i_{2} a_{1}$. Since $i_{1} \neq i_{2}$ and $1 \leqslant i_{1}, i_{2} \leqslant n-1$, we may assume that $w_{1}<z_{1}+(n$ - 2) $a_{1}$. But $[f] \cdot A_{i} \leqslant 0$ for all $A_{i} \subseteq A$ by p. 133 of [2]. So $[f]>Z$, by the definition of fundamental cycle $Z$. In particular, $z_{1}<a_{1}$. So, $w_{1}<(n-1) a_{1}$. This contradicts (3.1). Q.E.D.

A partial converse of Theorem 3.2 will be proved later. Weakly elliptic singularities can be effectively studied by the following method of elliptic sequences.

DEFINITION 3.3. Let $A$ be the exceptional set of the minimal good resolution $\pi: M \rightarrow V$ where $V$ is a normal two-dimensional Stein space with $p$ as its only weakly elliptic singularity. If $E \cdot Z<0$, we say that the elliptic sequence is $\{Z\}$ and the length of elliptic sequence is equal to one. Suppose $E \cdot Z=0$. Let $B_{1}$ be the maximal connected subvariety of $A$ such that $B_{1} \supseteq$ supp $E$ and $A_{i} \cdot Z=0 \forall A_{i} \subseteq B_{1}$. Since $A$ is an exceptional set, $Z \cdot Z<0$. So $B_{1}$ is properly contained in $A$. Suppose $Z_{B_{1}} \cdot E=0$. Let $B_{2}$ be the maximal connected subvariety of $B_{1}$ such that $B_{2} \supseteq|E|$ and $A_{i} \cdot Z_{B_{1}}=0 \forall A_{i} \subseteq B_{2}$. By the same argument as above, $B_{2}$ is properly contained in $B_{1}$. Continuing this 
process, we finally obtain $B_{m}$ with $Z_{B_{m}} \cdot E<0$. We call $\left\{Z_{B_{0}}=Z\right.$, $\left.Z_{B_{1}}, \ldots, Z_{B_{m}}\right\}$ the elliptic sequence and the length of elliptic sequence is $m+1$.

EXAMPLE 1. Let $p$ be a weakly elliptic singularity whose weighted dual graph is of the following form:

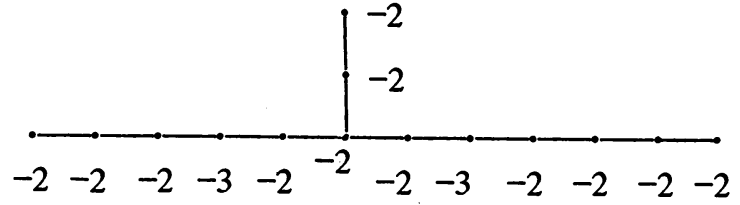

$$
\begin{aligned}
& 1 \\
& \begin{array}{llllllllllll}
Z=1 & 1 & 1 & 1 & 2 & 3 & 2 & 1 & 1 & 1 & 1 & 1
\end{array} \\
& \begin{array}{lllllllllllll} 
& 1 \\
Z_{B_{1}}= & 0 & 1 & 1 & 1 & 2 & 3 & 2 & 1 & 1 & 1 & 1 & 0
\end{array} \\
& \begin{array}{lllllllllllll} 
& 1 \\
Z_{B_{2}}= & 0 & 0 & 1 & 1 & 2 & 3 & 2 & 1 & 1 & 1 & 0 & 0
\end{array} \\
& Z_{B_{3}}=\begin{array}{llllllllllll}
0 & 0 & 0 & 1 & 2 & 3 & 2 & 1 & 1 & 0 & 0 & 0
\end{array} \\
& E=0 \begin{array}{llllllllllll} 
& 0 & 0 & 1 & 2 & 3 & 2 & 1 & 0 & 0 & 0 & 0
\end{array}
\end{aligned}
$$

The elliptic sequence is $\left\{Z=Z_{B_{0}}, Z_{B_{1}}, Z_{B_{2}}, Z_{B_{3}}\right\}$ and the length of elliptic sequence is 4.

REMARK 3.4. The elliptic sequence is defined purely topologically.

EXAMPLE 2. Let $p$ be a weakly elliptic singularity whose weighted dual graph is of the following form:

$$
\begin{aligned}
& \underset{-2-2-2-3}{\sim} \\
& \begin{array}{lllllllll}
Z=1 & 1 & 1 & 1 & 1 & 1 & 1 & 1 & 1
\end{array} \\
& Z_{B_{1}}=\begin{array}{llllllllll}
0 & 1 & 1 & 1 & 1 & 1 & 1 & 1 & 0
\end{array} \\
& Z_{B_{2}}=\begin{array}{llllllllll}
0 & 0 & 1 & 1 & 1 & 1 & 1 & 0 & 0
\end{array} \\
& Z_{B_{3}}=\begin{array}{llllllllll}
0 & 0 & 0 & 1 & 1 & 1 & 0 & 0 & 0
\end{array}
\end{aligned}
$$


The elliptic sequence is $\left\{Z=Z_{B_{0}}, Z_{B_{1}}, Z_{B_{2}}, Z_{B_{3}}=Z_{E}\right\}$ and the length of the elliptic sequence is 4 .

Proposition 3.5. Let $\pi: M \rightarrow V$ be the minimal good resolution of a normal two-dimensional Stein space with $p$ as its only weakly elliptic singularity. Then for any $A_{i} \in|E|, A_{i}$ are nonsingular rational curves with self-intersection number less than or equal to -2 .

PROOF. The fact that $A_{i} \&|E|$ are nonsingular rational curves follows from Lemma 2.1. Suppose there exists $A_{i} Z|E|$ such that $A_{i} \cdot A_{i}=-1$. It follows easily from Proposition 2.2 that $A_{i}$ is a "star" in the dual weighted graph $\Gamma$ of exceptional set $\pi^{-1}(p)=A$, i.e., there exist $A_{1}, A_{2}, A_{3} \subseteq A$ such that $\Gamma$ has the following graph as its subgraph:

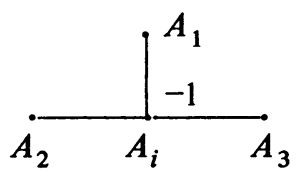

or there exist $A_{1} \subseteq A$ such that $\Gamma$ has the following graph as its subgraph:

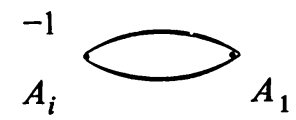

Then $\chi\left(A_{1}+A_{2}+A_{3}+2 A_{i}\right)=0$ in the former case, and $\chi\left(A_{1}+2 A_{i}\right)=0$ in the latter case. These are impossible by Proposition 1.7 and that $A_{i} Z$ $\operatorname{supp} E$.

LEMMA 3.6. Let $\Gamma$ be a weighted dual graph including genera for the vertices associated to the minimal good resolution of the normal two-dimensional weakly elliptic singularity $p$. Suppose $p$ is not a minimally elliptic singularity; then $-K^{\prime}>Z+E$ whenever $K^{\prime}$ exists. If $E \cdot Z<0$ and $|E| \subset A$, then $K^{\prime}$ does not exist.

Proof. If $\pi$ is the minimal resolution, then $A_{i} \cdot K^{\prime}>0$ for all $A_{i} \subseteq A$ by Proposition 1.2. So $-K^{\prime}>Z>E$ by the definition of fundamental cycle. Suppose $\pi$ is not the minimal resolution. Then the corresponding dual weighted graph $\Gamma$ consists of either

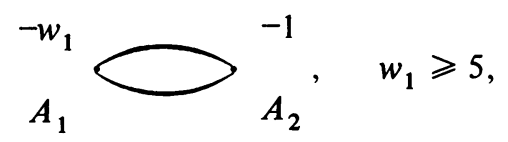

or

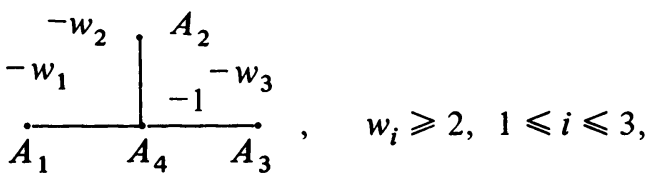


as its proper subgraph. In case (a), $E=A_{1}+A_{2}$. We claim that $k_{1}^{\prime} \neq 0$ where $K^{\prime}=\sum k_{i}^{\prime} A_{i}$. For if $k_{1}^{\prime}=0$, then $A_{1} \cdot K^{\prime} \leqslant 0$ since $K^{\prime}$ is a negative cycle. On the other hand,

$$
A_{1} \cdot K^{\prime}=A_{1} \cdot K=-A_{1} \cdot A_{1}+2 g_{1}-2>3>0 .
$$

This is a contradiction. Hence $k_{1}^{\prime} \neq 0$. We claim that $k_{2}^{\prime}$ cannot also be zero. For if $k_{2}^{\prime}=0$, then $A_{2} \cdot K^{\prime} \leqslant-2$ since $A_{2} \cdot A_{1}=2$. On the other hand,

$$
A_{2} \cdot K^{\prime}=A_{2} \cdot K=-A_{2} \cdot A_{2}+2 g_{2}-2=1-2=-1 \text {. }
$$

This is a contradiction. Hence, $k_{2}^{\prime} \neq 0$. It follows that $-K^{\prime}>E$. In case (b), $E=A_{1}+A_{2}+A_{3}+2 A_{4}$. We claim that one of $k_{i}^{\prime}, 1<i<3$, cannot equal zero. For if $k_{1}^{\prime}=k_{2}^{\prime}=k_{3}^{\prime}=0$, then $A_{4} \cdot K^{\prime} \geqslant 0$. This is because there exists no $A_{i} Z|E|, A_{i} \cdot A_{4}>0$ by the proof of Proposition 3.5. However,

$$
A_{4} \cdot K^{\prime}=A_{4} \cdot K=-A_{4} \cdot A_{4}+2 g_{4}-2=1-2=-1 \text {. }
$$

This is a contradiction. So we may assume $k_{1}^{\prime} \neq 0$. If $k_{2}^{\prime}=0$, then $A_{2} \cdot K^{\prime}<$ 0 . On the other hand,

$$
A_{2} \cdot K^{\prime}=A_{2} \cdot K=-A_{2} \cdot A_{2}-2>0 .
$$

Hence, $A_{2} \cdot K^{\prime}=0$ and $A_{2} \cdot A_{2}=-2$. If $k_{3}^{\prime}$ also equals 0 , then a similar argument will show $A_{3} \cdot A_{3}=-2$. The intersection matrix cannot be negative definite. So we may assume that $k_{3}^{\prime} \neq 0$. We claim that $k_{4}^{\prime} \neq 0$. For if $k_{4}^{\prime}=0$, then $A_{4} \cdot\left(K^{\prime}\right) \leqslant-2$. On the other hand,

$$
A_{4} \cdot K^{\prime}=A_{4} \cdot K=A_{4} \cdot A_{4}+2 g_{4}-2=-1 \text {. }
$$

This is a contradiction. So $k_{4}^{\prime} \neq 0$. We claim that $k_{2}^{\prime} \neq 0$. For if $k_{2}^{\prime}=0$, then $A_{2} \cdot\left(K^{\prime}\right) \leqslant-1$. On the other hand,

$$
A_{2} \cdot K^{\prime}=A_{2} \cdot K=A_{2} \cdot A_{2}+2 g_{2}-2>0 .
$$

This is a contradiction. We claim that $k_{4}^{\prime}<-2$. For if $k_{4}^{\prime}=-1$, then $A_{4} \cdot K^{\prime}=k_{1}^{\prime}+k_{2}^{\prime}+k_{3}^{\prime}+1 \leqslant-2$. On the other hand,

$$
A_{4} \cdot K^{\prime}=A_{4} \cdot K=-A_{4} \cdot A_{4}+2 g_{4}-2=-1 \text {. }
$$

This is a contradiction. So $k_{4}^{\prime} \leqslant-2$. We have proved in both cases (a) and (b) $-K^{\prime}>E$. We claim that actually $-K^{\prime} \gg E$. Since $p$ is not a minimally elliptic singularity, there exists $A_{i} \&|E|, A_{i}^{\neq} \cap E \neq \varnothing$. It suffices to prove $k_{i}^{\prime} \neq 0$. For if $k_{i}^{\prime}=0$, then $A_{i} \cdot K^{\prime}<0$. On the other hand,

$$
A_{i} \cdot K^{\prime}=A_{i} \cdot K=-A_{i} \cdot A_{i}+2 g_{i}-2=-A_{i} \cdot A_{i}-2 \geqslant 0 .
$$

This is a contradiction. Therefore $-K^{\prime}=E+D$ where $D$ is a nonzero positive cycle. We claim that $A_{i} \cdot D \leqslant 0$ for all $A_{i} \subseteq A$. If $A_{i} \subseteq|E|$, then $A_{i}\left(-K^{\prime}\right)=A_{i}(-K)=A_{i} \cdot E$ by Lemma 1.8. So $A_{i} \cdot D=0$. If $A_{i} \&|E|$, then $A_{i} \cdot A_{i} \leqslant-2$ and, hence,

$$
A_{i}\left(-K^{\prime}\right)=A_{i}(-K)=A_{i} \cdot A_{i}+2<0 .
$$

However, $A_{i} \&|E|$, so $A_{i} \cdot E \geqslant 0$. It follows that $A_{i} \cdot D=A_{i}\left(-K^{\prime}\right)-A_{i} E<$ 0 . This proves our claim. By definition of the fundamental cycle, $D>Z$. So, in particular, $-K^{\prime} \geqslant Z+E$.

Suppose $E \cdot Z<0$, we want to prove $K^{\prime}$ does not exist. Suppose on the contrary that $K^{\prime}$ exists. By the above proof, $-K^{\prime}=Z+D$ where $D$ is a 
positive cycle. By (1.2),

$$
\begin{aligned}
\chi\left(-K^{\prime}\right) & =-\frac{1}{2}\left[\left(-K^{\prime}\right) \cdot K+\left(-K^{\prime}\right) \cdot\left(-K^{\prime}\right)\right] \\
& =-\frac{1}{2}\left[\left(-K^{\prime}\right) \cdot\left(K^{\prime}\right)+K^{\prime} \cdot K^{\prime}\right]=0,
\end{aligned}
$$

so $0=\chi(Z+D)=\chi(Z)+\chi(D)-Z \cdot D$. Since $p$ is a weakly elliptic singularity, $\chi(Z) \geqslant 0$ and $\chi(D) \geqslant 0$. Also $Z \cdot D \leqslant 0$ because $Z$ is the fundamental cycle and $D$ is a positive cycle. It follows easily that $\chi(D)=0$ and $Z \cdot D=0$. Since $Z \cdot E<0,|D| \beth|E|$. By Proposition 1.7, we conclude that $D=0$. But then $Z=-K^{\prime} \geqslant Z+E$, which is absurd.

THEOREM 3.7. Let $\pi: M \rightarrow V$ be the minimal good resolution of a normal two-dimensional Stein space with $p$ as its only weakly elliptic singularity. Suppose $p$ is not a minimally elliptic singularity and $K^{\prime}$ exists. Then the elliptic sequence is of the following form:

$$
Z_{B_{0}}=Z, Z_{B_{1}}, \ldots, Z_{B_{l}}, Z_{B_{l+1}}=Z_{E}, \quad l \geqslant 0 .
$$

Moreover, $-K^{\prime}=\sum_{i=0}^{l} Z_{B_{i}}+E$.

Proof. Lemma 3.6 says that length of the elliptic sequence is greater than or equal to 2 and $-K^{\prime}>Z+E$. So $-K^{\prime}=Z+D_{1}$ where $D_{1}$ is a nonzero positive cycle on $A$. By $(1.2), \chi\left(-K^{\prime}\right)=0$. So $0=\chi\left(Z+D_{1}\right)=\chi(Z)+$ $\chi\left(D_{1}\right)-Z \cdot D_{1}$. Since $p$ is a weakly elliptic singularity, $\chi(Z)=0, \chi\left(D_{1}\right)>0$. Because $Z$ is the fundamental cycle and $D_{1}$ is a positive cycle, so $Z \cdot D_{1} \leqslant 0$. Consequently, $\chi\left(D_{1}\right)=0$ and $Z \cdot D_{1}=0$. By Proposition 1.7, $\chi\left(D_{1}\right)=0$ implies that $\left|D_{1}\right|$ is connected and contains $|E|$. We claim that $\left|D_{1}\right|=B_{1}$. Since $D_{1} \cdot Z=0$ and $\left|D_{1}\right|$ is connected and contains $|E|$, we have $\left|D_{1}\right| \subseteq B_{1}$. Suppose $\left|D_{1}\right| \neq B_{1}$. Then there exists $A_{i} \_\left|D_{1}\right|, A_{i} \subseteq B_{1}$ and $A_{i} \cap\left|D_{1}\right| \neq \varnothing$. Hence, $A_{i}\left(-K^{\prime}\right)=A_{i}\left(Z+D_{1}\right)=A_{i} \cdot D_{1}>0$. On the other hand, since $A_{i} Z$ $|E|, A_{i} \cdot\left(-K^{\prime}\right)=A_{i} \cdot A_{i}+2 \leqslant 0$, by Proposition 3.5. This is a contradiction. Hence, $\left|B_{1}\right|=\left|D_{1}\right|$. Let $U_{1}$ be a holomorphically convex neighborhood of $B_{1}$ such that $\Phi_{1}: U_{1} \rightarrow V_{1}$ represents $B_{1}$ as exceptional set where $V_{1}$ is a normal two-dimensional Stein space with $\Phi_{1}\left(B_{1}\right)$ as its only singularity. We claim that the $K^{\prime}$ cycle on $U_{1}$ which is denoted by $K_{U_{1}}^{\prime}$ exists and $K_{U_{1}}^{\prime}=-D_{1}$. In fact for any $A \subseteq B_{1}$,

$$
\begin{aligned}
A_{i} \cdot\left(-D_{1}\right) & =A_{i} \cdot\left(-Z-D_{1}\right) \\
& =A_{i} \cdot K \quad \text { where } K \text { is the canonical divisor on } M \\
& =-A_{i} \cdot A_{i}+2 g_{i}-2 \\
& =A_{i} \cdot K_{U_{1}} \quad \text { where } K_{U_{1}} \text { is the canonical divisor on } U_{1} .
\end{aligned}
$$

So $-D_{1}=K_{U_{1}}^{\prime}$. By induction on the length of elliptic sequence, the proof reduces to the following proposition.

Proposition 3.8. Let $\pi: M \rightarrow V$ be the minimal good resolution of a normal two-dimensional Stein space with $p$ as its only weakly elliptic singularity. Suppose $p$ is not a minimally elliptic singularity and $K^{\prime}$ exists. If the length of the elliptic sequence is equal to two, then the elliptic sequence is $\left\{Z, Z_{E}\right\}$. Moreover $-K^{\prime}=Z+E$. 
Proof. Lemma 3.6 says that $-K^{\prime}>Z$. So $-K^{\prime}=Z+D$ where $D$ is a nonzero positive cycle on $A$. By (1.2), $\chi\left(-K^{\prime}\right)=0$. So $0=\chi(Z+D)=\chi(Z)$ $+\chi(D)-Z \cdot D$. Since $p$ is a weakly elliptic singularity, $\chi(Z)=0$ and $\chi(D)>0$. As $Z$ is the fundamental cycle and $D$ is a positive cycle, we have $Z \cdot D \leqslant 0$. Consequently, $\chi(D)=0$ and $Z \cdot D=0$. Arguing as above, we know that $\left|B_{1}\right|=|D|$. Moreover $K_{U_{1}}^{\prime}$ exists and $K_{U_{1}}^{\prime}=-D$ where $U_{1}$ is a holomorphic convex neighborhood of $B_{1}$. By Lemma 3.6, $B_{1} \neq|E|$ cannot occur since the length of the elliptic sequence is equal to two. So $|D|=B_{1}=$ $|E|$. We claim that $D=E$. Since $\chi(D)=0$, we have $D \geqslant E$, i.e., $D=E+$ $D^{\prime}$, where $D^{\prime}$ is a positive cycle with $\left|D^{\prime}\right| \subseteq|E|$. Since $A_{i} \cdot D=A_{i} \cdot\left(-K_{U_{1}}^{\prime}\right)$ $=A_{i} \cdot E$ for all $A_{i} \subseteq|E|$, so $A_{i} \cdot D^{\prime}=0$ for all $A_{i} \subseteq|E|$. It follows that $D^{\prime} \cdot D^{\prime}=0$. Therefore $D^{\prime}=0$ and $D=E$. We have proved the elliptic sequence is $\left\{Z, Z_{E}\right\}$ and $-K^{\prime}=Z+E$. Q.E.D.

Let $p$ be the only singularity of the two-dimensional hypersurface Stein space $V$. Let $\pi: M \rightarrow V$ be a resolution of $V$. Let $A=\cup_{i} A_{i}, 1<i<n$, be the decomposition of $A=\pi^{-1}(p)$ into irreducible components. Let $\mu$ be the Milnor number of $p$. Then Laufer [23] proved that

$$
\mu=n+K^{\prime} \cdot K^{\prime}-\operatorname{dim} H^{1}(A ; \mathbf{C})+12 \operatorname{dim} H^{1}(M, \vartheta) .
$$

(3.2) has various applications. One is that it gives a means of calculating $\operatorname{dim} H^{1}(M, \theta)$ for hypersurface singularities. This calculation is very difficult, if not impossible, in general. However, given a weighted dual graph corresponding to a singularity, we have to solve a system of linear equations in order to find $K^{\prime}$. For weakly elliptic singularities, Theorem 3.7 provides us a quick method to find $K^{\prime}$.

2. Maximally elliptic singularities. The length of the elliptic sequence gives information about $h=\operatorname{dim} H^{1}(M, \mathcal{\theta})$.

THEOREM 3.9. Let $\pi: M \rightarrow V$ be the minimal good resolution of a normal two-dimensional Stein space with $p$ as its only weakly elliptic singularity. Then $\operatorname{dim} H^{1}(M, \theta)$ is less than or equal to the length of the elliptic sequence if $K^{\prime}$ exists.

Proof. If the length of the elliptic sequence is equal to 1 , i.e. the elliptic sequence consists of the fundamental cycle $Z$ only, then $Z \cdot E<0$. By Theorem 4.1 of [24], $H^{1}(M, \theta)=C$. So from now on, we may assume that the elliptic sequence is of the following form:

$$
Z_{B_{0}}=Z, Z_{B_{1}}, \ldots, Z_{B_{l}}, Z_{B_{l+1}}=Z_{E^{\prime}}, \quad l \geqslant 0,
$$

and $K^{\prime}=-\left(\sum_{i=0}^{l} Z_{B_{i}}+E\right)$ by Theorem 3.7. Choose a computation sequence for the fundamental cycle $Z$ of the following form:

$$
\begin{aligned}
Z_{0}=0, Z_{1}, \ldots, Z_{k}=E, \ldots, Z_{r_{1}} & =Z_{B_{l}}, \ldots, \\
Z_{r_{i}} & =Z_{B_{l-j+1}}, \ldots, Z_{r_{l+1}}=Z_{B_{0}}=Z .
\end{aligned}
$$


Consider the following sheaf exact sequence:

$$
\begin{aligned}
& 0 \rightarrow \theta(-Z) / \theta\left(-Z-Z_{1}\right) \rightarrow \theta_{Z+Z_{1}} \rightarrow \theta_{Z} \rightarrow 0 \\
& 0 \rightarrow \mathcal{\theta}\left(-Z-Z_{k-1}\right) / \mathcal{O}(-Z-E) \rightarrow \theta_{Z+E} \rightarrow \theta_{Z+Z_{k-1}} \rightarrow 0 \\
& 0 \rightarrow \mathcal{\theta}\left(-Z-Z_{r_{l}-1}\right) / \mathcal{\theta}\left(-Z-Z_{r_{l}}\right) \rightarrow \theta_{Z+Z_{B_{1}}} \rightarrow \theta_{Z+Z_{r_{l}-1}} \rightarrow 0 \\
& 0 \rightarrow \Theta\left(-\sum_{i=0}^{j} Z_{B_{i}}\right) / \Theta\left(-\sum_{i=0}^{j} Z_{B_{i}}-Z_{1}\right) \rightarrow \Theta_{\Sigma_{i-0}^{j} Z_{B_{i}}+Z_{1}} \rightarrow \Theta_{\Sigma_{i-0}^{j} Z_{B_{i}}} \rightarrow 0 \\
& 0 \rightarrow \Theta\left(-\sum_{i=0}^{j} Z_{B_{i}}-Z_{k-1}\right) / \Theta\left(-\sum_{i=0}^{j} Z_{B_{i}}-E\right) \rightarrow \Theta_{\sum_{i=0}^{j} Z_{B_{i}}}+E \\
& \rightarrow \vartheta_{\Sigma_{i-0}^{j} z_{B_{i}}}+z_{k-1} \rightarrow 0 \\
& 0 \rightarrow \mathcal{\Theta}\left(-\sum_{i=0}^{j} Z_{B_{i}}-Z_{r_{t-j}-1}\right) / \mathcal{O}\left(-\sum_{i=0}^{j} Z_{B_{i}}-Z_{B_{j+1}}\right) \\
& \rightarrow \mathcal{O}_{\Sigma_{i-0}^{j} z_{B_{i}}}+z_{B_{j+1}} \rightarrow \mathcal{O}_{\sum_{i-0}^{j} z_{B_{i}}+z_{r_{l-j}-1}} \rightarrow 0
\end{aligned}
$$

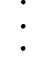

$$
\begin{aligned}
& 0 \rightarrow \mathcal{O}\left(-\sum_{i=0}^{l} Z_{B_{i}}\right) / \mathcal{O}\left(-\sum_{i=0}^{l} Z_{B_{i}}-Z_{1}\right) \rightarrow \Theta_{\Sigma_{i-0}^{\prime} Z_{B_{i}}}+Z_{1} \\
& \rightarrow \mathcal{O}_{\Sigma_{i-0}^{\prime} Z_{B_{i}}} \rightarrow 0 \\
& 0 \rightarrow \Theta\left(-\sum_{i=0}^{l} Z_{B_{i}}-Z_{k-1}\right) / \theta\left(-\sum_{i=0}^{l} Z_{B_{i}}-E\right) \\
& \rightarrow \mathcal{O}_{\Sigma_{i-0}^{\prime} Z_{B_{i}}+E} \rightarrow \mathcal{O}_{\Sigma_{i-0}^{\prime} Z_{B_{i}}+Z_{k-1}} \rightarrow 0 \text {. }
\end{aligned}
$$


Let $Z_{B_{i}}=\Sigma_{j B_{i}} z_{j} A_{j}$. We remark that if $E=A_{1}$ is an elliptic curve, then ${ }_{B_{i}} z_{1}=1$ for all $i$ by Corollary 2.6. The usual long cohomology exact sequence or (3.3), (1.6) and Reimann-Roch Theorem will show that

$\operatorname{dim} H^{1}\left(M, \Theta_{\Sigma_{i-0}^{\prime} Z_{B_{i}}+E}\right)<l+2=$ length of the elliptic sequence because

$$
H^{0}\left[M, \frac{\theta\left(-\Sigma_{i=0}^{j} Z_{B_{i}}-Z_{h-1}\right)}{\theta\left(-\Sigma_{i=0}^{j} Z_{B_{i}}-Z_{h}\right)}\right] \text { and } H^{1}\left[M, \frac{\theta\left(-\Sigma_{i=0}^{j} Z_{B_{i}}-Z_{h-1}\right)}{\theta\left(-\Sigma_{i=0}^{j} Z_{B_{i}}-Z_{h}\right)}\right]
$$

are nonzero only if $h=k$. Since

$$
H^{1}\left[M, \mathcal{O}\left(-\sum_{i=0}^{l} Z_{B_{i}}-E\right)\right]=H^{1}\left(M, \mathcal{O}\left(K^{\prime}\right)\right)=0
$$

by Corollary 3.3 of [20], the exact sequence

$$
H^{1}\left(M, \theta\left(-\sum_{i=0}^{l} Z_{B_{i}}-E\right)\right] \rightarrow H^{1}(M, \theta) \rightarrow H^{1}\left(M, \theta_{\Sigma_{l-0}^{\prime} Z_{B_{i}}+E}\right) \rightarrow 0
$$

shows that

$$
\operatorname{dim} H^{1}(M, \mathcal{O})=\operatorname{dim} H^{1}\left(M, \mathcal{O}_{\Sigma_{i-0}^{\prime} Z_{B_{i}}+E}\right)<l+2 \text {. Q.E.D. }
$$

The following example, which is due to Laufer, shows that $\operatorname{dim} H^{1}(M, \theta)$ can be strictly less than the length of the elliptic sequence even for hypersurface singularities. As far as the author's knowledge is concerned, this is the first known example for double-point singularity with maximal ideal cycle strictly greater than the fundamental cycle.

EXAMPLE 3 (LAUFER). Let $V$ be the locus in $C^{3}$ of $z^{2}=y\left(x^{4}+y^{6}\right)$. Then the dual weighted graph is

$$
\underset{\text { torus }}{-1 \quad-2}-2
$$

This is a weakly elliptic singualrity and the length of the elliptic sequence is equal to three. It can be calculated that $\mu=22$, where $\mu$ is the Milnor number. By Theorem 3.7,

$$
-K^{\prime}=Z_{B_{0}}+Z_{B_{1}}+E, \quad K^{\prime} \cdot K^{\prime}=Z_{B_{0}}^{2}+Z_{B_{1}}^{2}+E^{2}=-3 .
$$

By (3.2), we know $\operatorname{dim} H^{1}(M, \theta)=2$.

The following two examples show that $\operatorname{dim} H^{1}(M, \theta)$ can actually equal the length of the elliptic sequence. 
Example 4. Let $V$ be the locus in $C^{3}$ of $z^{2}=y^{3}+x^{9+6 l}$. Then the dual weighted graph is

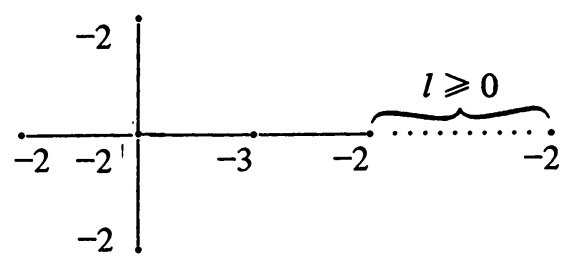

This is a weakly elliptic singularity and the length of the elliptic sequence is equal to $l+1$. It can be calculated that the Milnor number $\mu=16+12 l$. By Theorem 3.7,

$$
-K^{\prime}=\sum_{i=0}^{l-1} Z_{B_{i}}+E, \quad K^{\prime 2}=\sum_{i=0}^{l-1} Z_{B_{i}}^{2}+E^{2}=-(l+1) .
$$

By (3.2), we know that $\operatorname{dim} H^{1}(M, \theta)=l+1=$ length of the elliptic sequence.

EXAMPLE 5. Let $V$ be the locus in $C^{3}$ of $z^{2}=y^{3}+x^{11+6 l}$. Then the dual weighted graph is

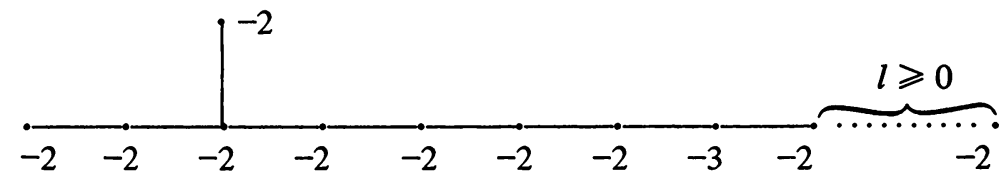

This is a weakly elliptic singularity and the length of the elliptic sequence is equal to $l+1$. It can be calculated that the Milnor number $\mu=20+12 l$. By Theorem 3.7,

$$
-K^{\prime}=\sum_{i=0}^{l-1} Z_{B_{i}}+E, \quad K^{\prime 2}=\sum_{i=0}^{l-1} Z_{B_{i}}^{2}+E^{2}=-(l+1)
$$

By (3.2), we know that $\operatorname{dim} H^{1}(M, \theta)=l+1=$ length of the elliptic sequence.

ExAmple 6 (WAgreich). Let $V$ be the locus in $C^{3}$ of $z^{3}=x^{3}+y^{3 l+1}$. Then the dual graph is

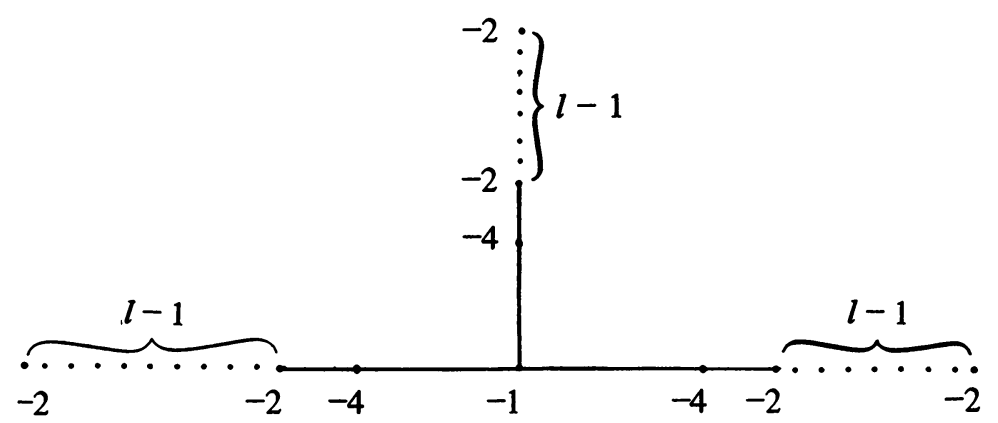


This is a weakly elliptic singularity and the length of the elliptic sequence is equal to $l$. It can be calculated that the Milnor number $\mu=12 l$. By Theorem 3.7, $-K^{\prime}=\sum_{i=0}^{l-1} Z_{B_{l}}+E, K^{\prime 2}=-(3 l+1)$. By (3.2) we know that $\operatorname{dim} H^{1}(M, \theta)=l=$ length of the elliptic sequence.

EXAmple 7 (Wagreich). Let $V$ be the locus in $C^{3}$ of $z^{3}=x^{3}+y^{3 l+2}$. Then the dual weighted graph is

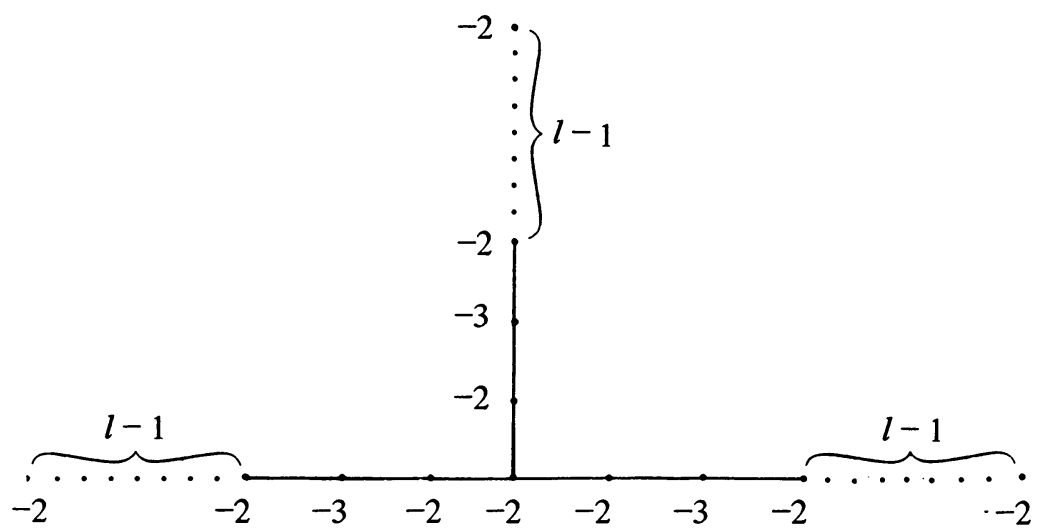

This is a weakly elliptic singularity and the length of the elliptic sequence is equal to $l$. It can be calculated that the Milnor number $\mu=12 l+4$. By Theorem 3.7, $-K^{\prime}=\sum_{i=0}^{l-1} Z_{B_{i}}+E, K^{\prime 2}=-3 l$. By (3.2) we know that $\operatorname{dim} H^{1}(M, \theta)=l=$ length of the elliptic sequence.

DEFINITION 3.10. Let $V$ be a normal 2-dimensional Stein space with $p$ as its only weakly elliptic singularity. Let $\pi: M \rightarrow V$ be the minimal good resolution. Suppose $K^{\prime}$ exists. If $\operatorname{dim} H^{1}(M, \theta)=$ length of the elliptic sequence, then $p$ is called a maximally elliptic singularity.

THEOREM 3.11. Let $\pi: M \rightarrow V$ be the minimal good resolution of a normal two-dimensional Stein space with $p$ as its only maximally elliptic singular point. Then ${ }_{v} \theta_{p}$ is Gorenstein.

Proof. If length of the elliptic sequence is equal to one, then Lemma 3.6 says that $p$ is a minimally elliptic singularity. By Theorem 3.10 of [24], ${ }_{v} \theta_{p}$ is Gorenstein. Therefore we may suppose that the length of the elliptic sequence is greater than or equal to two. By Theorem 3.7, we know that the elliptic sequence is of the following form:

$$
Z_{B_{0}}=Z, Z_{B_{1}}, \ldots, Z_{B_{l}}, Z_{B_{l+1}}=Z_{E}, l>0 \text { and }-K^{\prime}=\sum_{i=0}^{l} Z_{B_{i}}+E \text {. }
$$

Serre duality gives $H^{1}(M, \theta)$ as dual to $H_{*}^{1}(M, \Omega)$ where $\Omega$ is the canonical sheaf, i.e. the sheaf of germs of holomorphic 2-forms. By Theorem 3.4, p. 604 of [20], for suitable $M$, which can be arbitrarily small neighborhoods of $A=\pi^{-1}(p), H_{*}^{1}(M, \Omega)$ may be identified with $H^{0}(M-A, \Omega) / H^{0}(M, \Omega)$. 
Let $U_{1}$ be a holomorphically convex neighborhood of $B_{1}$ such that $\Phi_{1}$ : $U_{1} \rightarrow V_{1}$ represents $B_{1}$ as an exceptional set where $V_{1}$ is a normal twodimensional Stein space with $\Phi_{1}\left(B_{1}\right)$ as its only weakly elliptic singularity. We claim that $K_{U}$, the $K^{\prime}$ cycle on $U_{1}$, exists. In fact

$$
-K_{U_{1}}^{\prime}=Z_{B_{1}}+\cdots+Z_{B_{1}}+E \text {, }
$$

because for all $A_{i} \subseteq B_{1}$,

$$
\begin{aligned}
A_{i} \cdot\left(-\sum_{i=1}^{l} Z_{B_{i}}-E\right) & =A_{i}\left(-\sum_{i=0}^{l} Z_{B_{i}}-E\right) \\
& =A_{i} \cdot\left(K^{\prime}\right)=2 g_{i}-2-A_{i} \cdot A_{i} .
\end{aligned}
$$

So the length of the elliptic sequence relative to $\Phi_{1}$ is $l+1$. Let $\omega_{1}, \ldots, \omega_{l+2}$ $\in H^{0}(M-A, \Omega)$ such that its images form a basis for $H^{0}(M-$ $A, \Omega) / H^{0}(M, \Omega)$. Suppose, on the contrary, that ${ }_{\nu} \theta_{p}$ is not Gorenstein. We claim that the pole sets of $\omega_{i}, 1<i<l+2$, are contained in $B_{1}$. For if there exists $\omega_{i}$, say $\omega_{1}$, having a pole set which is not contained in $B_{1}$, then the divisor of $\omega_{1}$ has the following form:

$$
\left(\omega_{1}\right)=-\sum_{i=1}^{t} a_{i} A_{i}+\sum_{j=t+1}^{n} b_{j} A_{j}+\sum_{k=1}^{n_{1}} d_{k} X_{k}, \quad a_{i}>0, b_{j}>0, d_{k}>0,
$$

where $t>1, X_{r} \notin A, X_{r} \cap A \neq \varnothing, \forall 1<r<n_{1}$, and there exists $1<i<t$ such that $A_{i} \& B_{1}$. For any $A_{h} \subseteq A$,

$$
A_{h} \cdot\left(K^{\prime}\right)=A_{h} \cdot\left(\omega_{1}\right), \quad A_{h} \cdot\left(\left(\omega_{1}\right)-K^{\prime}\right)=0 .
$$

Let

$$
\left[\omega_{1}\right]=-\sum_{i=1}^{t} a_{i} A_{i}+\sum_{j=t+1}^{n} b_{j} A_{j}
$$

Then $A_{h} \cdot\left(\left[\omega_{1}\right]-K^{\prime}\right)<0$ for all $A_{h} \subseteq A$. Since ${ }_{\nu} \theta_{p}$ is not a Gorenstein ring, either there exists $0<b_{j}, t+1<j<n$, or there exists $d_{k}>0,0<k<n_{1}$, by Lemma 3.6. If the former case occurs, then $\left[\omega_{1}\right]-K^{\prime} \neq 0$ because $K^{\prime}$ is a negative cycle. If the latter case occurs, we claim that $\left[\omega_{1}\right]-K^{\prime} \neq 0$ also. For let $0<r<n_{1}$ such that $d_{r}>0$. There exists $A_{r} \subseteq A$ such that $A_{r} \cdot X_{r}>0$. Then

$$
\begin{aligned}
A_{r} \cdot\left(\left[\omega_{1}\right]-K^{\prime}\right) & =A_{r} \cdot\left[\omega_{1}\right]-A_{r} \cdot K^{\prime}=A_{r} \cdot\left[\omega_{1}\right]-A_{r} \cdot\left(\omega_{1}\right) \\
& =A_{r} \cdot\left[\omega_{1}\right]-A_{r} \cdot\left(\left[\omega_{1}\right]+\sum_{k=0}^{n_{1}} d_{k} X_{k}\right) \\
& =-A_{r} \cdot \sum_{k=0}^{n} d_{k} X_{k}<-d_{r}<0 .
\end{aligned}
$$

Therefore $\left[\omega_{1}\right]-K^{\prime}$ is not zero in any cases. Notice that some coefficient of $A_{i} \& B_{1}$ in $\left[\omega_{1}\right]-K^{\prime}$ is strictly less than the corresponding coefficient of that component in the fundamental cycle $Z$ because $-K^{\prime}=\sum_{i=0}^{l} Z_{B_{i}}+E$. If 
$\left[\omega_{1}\right]-K^{\prime}$ is a positive cycle, we let $Z^{1}=\inf \left(\left[\omega_{1}\right]-K^{\prime}, Z\right)$. It follows from M. Artin's argument, pp. 131-132 of [2] that $Z^{1}$ is also a positive cycle and $Z^{1} \cdot A_{k}<0$ for all $A_{k} \subseteq A$. However, $Z^{1}<Z$. This contradicts the definition of the fundamental cycle $Z$. So $\left[\omega_{1}\right]-K^{\prime}$ cannot be a positive cycle. Let

$$
Z_{0}=\left[\omega_{1}\right]-K^{\prime}=\sum_{i=1}^{s} h_{i} A_{i}-\sum_{j=s+1}^{n} c_{j} A_{j}, \quad h_{i}>0, c_{j}>0, s<n .
$$

Without loss of generality, we may assume that $c_{s+1}=\max \left(c_{j}\right), s+1<j<$ n. Consider

$$
Z_{1}=c_{s+1} Z+z_{s+1} Z_{0}=\sum_{i=0}^{n} z_{i}^{1} A_{i}
$$

where $Z=\sum_{i=1}^{n} z_{i} A_{i}$. Since $Z_{0} \cdot A_{i}<0$ for all $A_{i} \subseteq A$, we have $A_{i} \cdot Z_{1}<0$ for all $A_{i} \subseteq A$. Also $z_{i}^{1}>0$ for $1<i<s$ and $z_{s+1}^{1}=0$. By changing the index if necessary, we may assume $z_{s+2}^{1}=\min \left(z_{i}^{1}\right), s+2<i<n$. If $z_{s+2}^{1}>0$, then $Z_{1}$ is a positive cycle with $\operatorname{supp} Z_{1} \subset A$ because $z_{s+1}^{1}=0$. If $z_{s+2}^{1}<0$, consider

$$
Z_{2}=-z_{s+2}^{1} Z+z_{s+2} Z_{1}=\sum_{i=1}^{n} z_{i}^{2} A_{i}
$$

then $A_{i} \cdot Z_{2}<0$ for all $A_{i} \subseteq A, z_{i}^{2}>0$ for $1<i<s+1$ and $z_{s+2}^{2}=0$. Continuing this process, we finally get a positive cycle $D$ on $A$ with supp $D$ $\subset A$ and $A_{i} \cdot D<0$ for all $A_{i} \subseteq A$. But this is impossible by previous argument. We conclude that the pole set of $\omega_{i}, 1<i<l+2$, is contained in $B_{1}$. It follows that $\omega_{i} / U_{1}$, the restriction of $\omega_{i}$ to $U_{1}$, is in $H^{0}\left(U_{1}-B_{1}, \Omega\right)$ for all $1<i<l+2$. Since the length of the elliptic sequence on $U_{1}$ is $l+1$, by Theorem 3.9, $\operatorname{dim} H^{1}\left(U_{1}, \theta\right)<l+1$. Hence

$$
\operatorname{dim} H^{0}\left(U_{1}-B_{1}, \Omega\right) / H^{0}\left(U_{1}, \Omega\right)<l+1
$$

and there exist $\lambda_{1}, \ldots, \lambda_{l+2} \in \mathbf{C}$, not all $\lambda_{i}=0$, such that

$$
\lambda_{1} \omega_{1} / U_{1}+\cdots+\lambda_{l+2} \omega_{l+2} / U_{1} \in H^{0}\left(U_{1}, \Omega\right),
$$

where $\omega_{i} / U_{1}$ is the restriction of $\omega_{i}$ on $U_{1}$. It follows that

$$
\lambda_{1} \omega_{1}+\cdots+\lambda_{l+2} \omega_{l+2} \in H^{0}(M, \Omega),
$$

which contradicts our assumption that images of $\omega_{1}, \ldots, \omega_{l+2}$ form a basis for $H^{0}(M-A, \Omega) / H(M, \Omega)$.

THEOREM 3.12. Let $\pi: M \rightarrow V$ be the minimal good resolution of a normal two-dimensional Stein space with $p$ as its only maximally elliptic singularity. If $Z_{E} \cdot Z_{E}<-2$, then $m \theta=\theta(-Z)$.

Proof. If the length of the elliptic sequence is equal to one, then Lemma 3.6 says that $p$ is a minimally elliptic singularity. By [24] we have $m \theta=$ $\theta(-Z)$. From now on, we assume that the length of the elliptic sequence is greater than one. By Theorem 3.7, the elliptic sequence is of the form 


$$
Z_{B_{0}}=Z, Z_{B_{1}}, \ldots, Z_{B_{l}}, Z_{E}=Z_{B_{l+1}}, \quad l>0, \text { and }-K^{\prime}=\sum_{i=0}^{l} Z_{B_{i}}+E \text {. }
$$

Suppose $A_{1} \subseteq B_{1}$. We want to prove that

$$
H^{0}\left(M, \theta(-Z) / \theta\left(-Z-A_{1}\right)\right)=\mathbf{C}
$$

and

$$
H^{0}(M, \vartheta(-Z)) \rightarrow H^{0}\left(M, \vartheta(-Z) / \theta\left(-Z-A_{1}\right)\right)
$$

is surjective. We know that the Chern class of the line bundle associated to $\theta(-Z) / \mathcal{O}\left(-Z-A_{1}\right)$ is $-A_{1} \cdot Z=0$. By the Riemann-Roch Theorem,

$$
\operatorname{dim} H^{0}\left(M, \theta(-Z) / \theta\left(-Z-A_{1}\right)\right)<1 .
$$

In fact, $H^{0}\left(M, \theta(-Z) / \theta\left(-Z-A_{1}\right)\right)=0$ only if $A_{1}$ is an elliptic curve and $\theta(-Z) / \theta\left(-Z-A_{1}\right)$ is a sheaf of germs of sections of a nontrivial line bundle over $A_{1}$. Suppose, on the contrary, that $H^{0}(M, \vartheta(-Z) / \Theta(-Z-$ $\left.A_{1}\right)$ ) is not isomorphic to $\mathrm{C}$ or

$$
H^{0}(M, \vartheta(-Z)) \rightarrow H^{0}\left(M, \vartheta(-Z) / \vartheta\left(-Z-A_{1}\right)\right)
$$

is not surjective. Then

$$
H^{0}\left(M, \vartheta\left(-Z-A_{1}\right)\right) \rightarrow H^{0}(M, \vartheta(-Z))
$$

is an isomorphism. Choose a computation sequence for $Z$ as follows:

$$
Z_{0}=0, Z_{1}=A_{i_{1}}=A_{1}, \ldots, Z_{r_{1}}=Z_{B_{1}}, \ldots, Z_{r_{1+1}}=Z \text {. }
$$

Consider the following sheaf exact sequences.

$$
\begin{aligned}
& 0 \rightarrow \theta\left(-Z-Z_{2}\right) \rightarrow \theta\left(-Z-Z_{1}\right) \rightarrow \theta\left(-Z-Z_{1}\right) / \theta\left(-Z-Z_{2}\right) \rightarrow 0 \\
& 0 \rightarrow \theta\left(-Z-Z_{3}\right) \rightarrow \theta\left(-Z-Z_{2}\right) \rightarrow \theta\left(-Z-Z_{2}\right) / \theta\left(-Z-Z_{3}\right) \rightarrow 0 \\
& 0 \rightarrow \mathcal{U}\left(-Z-A_{B_{1}}\right) \rightarrow \mathcal{O}\left(-Z-A_{r_{l-1}}\right) \rightarrow \mathcal{\Theta}\left(-Z-Z_{r_{l-1}}\right) / \mathcal{O}\left(-Z-Z_{r_{l}}\right) \rightarrow 0 \text {. }
\end{aligned}
$$

The Chern classes of the line bundles associated to $\theta\left(-Z-Z_{i}\right) / \theta(-Z-$ $Z_{i+1}$ ) for $1<i<r_{l}-1$ are strictly less than zero. By the Riemann-Roch Theorem,

$$
H^{0}\left(M, \vartheta\left(-Z-Z_{i}\right) / \theta\left(-Z-Z_{i+1}\right)\right)=0 \text { for } 1<i<r_{l}-1 .
$$

The corresponding long coholomogy exact sequences of (3.4) will show that

$$
H^{0}\left(M, \theta\left(-Z-Z_{i+1}\right)\right) \rightarrow H^{0}\left(M, \vartheta\left(-Z-Z_{i}\right)\right)
$$

are isomorphisms, for $1<i<r_{l}-1$. By composing the maps, we get

$$
H^{0}\left(M, \theta\left(-Z-Z_{B_{1}}\right)\right) \rightarrow H^{0}(M, \theta(-Z))
$$

is an isomorphism. However, by [2], $m \theta \subset \theta(-Z)$. Therefore, if $g \in$ $H^{0}(M, m \theta)$, then $g \in H^{0}\left(M, \theta\left(-Z-Z_{B_{1}}\right)\right)$. Since ${ }_{v} \theta_{p}$ is Gorenstein, there exists $\omega \in H^{0}(M-A, \Omega)$ having no zeros near $A$. Serre duality gives 
$H^{1}(M, \theta)$ as dual to $H_{*}^{1}(M, \Omega)$, where $\Omega$ is the canonical sheaf, i.e., the sheaf of germs of holomorphic 2-forms. By Theorem 3.4, p. 604 of [20], for suitable $M$, which can be chosen to be arbitrarily small neighborhoods of $A=$ $\pi^{-1}(p), H_{*}^{1}(M, \Omega)$ may be identified with $H^{0}(M-A, \Omega) / H^{0}(M, \Omega)$. Since $p$ is the maximally elliptic singularity,

$$
\operatorname{dim} H^{0}(M-A, \Omega) / H^{0}(M, \Omega)=l+2
$$

and there exist $\omega_{1}, \ldots, \omega_{l+1} \in H^{0}(M-A, \Omega)$ such that images of $\omega_{1}, \ldots, \omega_{l+1}, \omega$ form a basis of $H^{0}(M-A, \Omega) / H^{0}(M, \Omega)$. As $\omega$ is nonzero in a neighborhood of $A$, we may assume that $\omega_{i}=f_{i} \omega$, where $f_{i} \in H^{0}(M, \theta)$. Moreover, we can assume that $f_{i}$ are vanishing at $p$; otherwise we need only replace $f_{i}$ by $f_{i}-f_{i}(p)$. Let $U_{1}$ be a holomorphic convex neighborhood of $B_{2}$ such that $\Phi: U_{1} \rightarrow V_{1}$ represents $B_{2}$ as an exceptional set where $V_{1}$ is a normal two-dimensional Stein space with $\Phi\left(B_{2}\right)$ as its only weakly elliptic singular point. Observe that the $K^{\prime}$ cycle on $U_{1}$ which is denoted by $K_{U_{1}}^{\prime}$ is equal to $\sum_{i=2}^{l} Z_{B_{i}}+E$. Since in this case, the length of the elliptic sequence is $l$ by Theorem 3.9, $\operatorname{dim} H^{1}\left(U_{1}, \theta\right) \leqslant l$. On the other hand, as $(\omega)=\sum_{i=0}^{l} Z_{B_{i}}+$ $E$ and $H^{0}(M, m \theta) \subseteq H^{0}\left(M, \theta\left(-Z-Z_{B_{1}}\right)\right)$, we can restrict $\omega_{1}=$ $f_{1} \omega, \ldots, \omega_{l+1}=f_{l+1} \omega$ to $U_{1}$ and get $\left\{\omega_{1} / U_{1}, \ldots, \omega_{l+1} / U_{1}\right\}$ which is linearly independent in $H^{0}\left(U_{1}-B_{2}, \Omega\right) / H^{0}\left(U_{1}, \Omega\right)$. But this is impossible since $\operatorname{dim} H_{*}^{1}(M, \theta) \leqslant l$. So we conclude that

$$
H^{0}\left(M, \vartheta(-Z) / \vartheta\left(-Z-A_{1}\right)\right)=\mathbf{C}
$$

and

$$
H^{0}(M, \vartheta(-Z)) \rightarrow H^{0}\left(M, \vartheta(-Z) / \vartheta\left(-Z-A_{1}\right)\right)
$$

is surjective. Given a point $a \in A_{1}$, let

$$
\tilde{f} \in H^{0}\left(M, \Theta(-Z) / \theta\left(-Z-A_{1}\right)\right)
$$

be nonzero near $a$ as a section of the line bundle. $f \in H^{0}(M, \theta(-Z))$ projecting onto $\tilde{f}$ will generate $\mathcal{\theta}(-Z)$ near $a$ since it must vanish to the prescribed order on $A_{1}$ near $a$ and will have no other zeros near $a$.

In order to prove $\theta(-Z) \subseteq m$, it remains to prove $\theta(-Z) \subseteq m \theta$ near $A-B_{1}$. There are two subcases.

Case (i). There exists $A_{i} \subseteq \operatorname{supp} E$ such that $E \cdot Z_{E}+1<A_{1} \cdot Z_{E}<-1$ or $E=A_{i}$ is a nonsingular elliptic curve. For any $A_{1} Z$ supp $E$, choose a computation sequence for the fundamental cycle $Z$ of the following form:

$$
Z_{0}=0, Z_{1}=A_{i_{1}}=A_{1}, \ldots, Z_{r}, Z_{r+1}, \ldots, Z_{r+k}=Z_{r}+E, \ldots, Z_{r_{l+1}}=Z \text {, }
$$

in which supp $Z_{r} \subseteq \overline{\operatorname{supp} Z-\operatorname{supp} E}$ and $Z_{r+1}-Z_{r}, \ldots, Z_{r+k}-Z_{r}=E$ is part of a computation sequence for $Z$. If $\operatorname{supp} E$ has at least two irreducible components, then our hypothesis guarantees that the computation sequence can be so chosen such that $A_{i_{r+k}} \cdot Z_{E}<0$ by Proposition 2.5. Consider the 
following sheaf exact sequence for $n>0$ :

Diagram A (page 299).

We claim that

$$
\left(\sum_{j=0}^{l} Z_{B_{j}}+Z_{E}\right) \cdot A_{i}<0 \text { for all } A_{i} \subseteq A
$$

It is obvious that

$$
A_{i} \cdot\left(\sum_{j=0}^{l} Z_{B_{j}}+Z_{E}\right)<0 \text { for } A_{i} \subseteq \operatorname{supp} E .
$$

If $A_{i} \&$ supp $E$, then $A_{i} \cdot Z_{E}=A_{i} \cdot E$ by Proposition 2.5 . Hence

$$
\begin{aligned}
A_{i} \cdot\left(\sum_{j=0}^{l} Z_{B_{j}}+Z_{E}\right) & =A_{i} \cdot\left(\sum_{j=0}^{l} Z_{B_{j}}+E\right) \\
& =A_{i} \cdot\left(-K^{\prime}\right)=-A_{i} \cdot K=2+A_{i} \cdot A_{i}<0 \text { for } A_{i} \text { \&upp } E .
\end{aligned}
$$

This proves our claim.

$$
\vartheta\left(-\sum_{i=0}^{l} Z_{B_{i}}-Z_{E}-n Z-Z_{j-1}\right) / \mathcal{O}\left(-\sum_{i=0}^{l} Z_{B_{i}}-Z_{E}-n Z-Z_{j}\right)
$$

is the sheaf of germs of sections of a line bundle over $A_{i}$ of Chern class $-A_{i_{j}} \cdot\left(\sum_{i=0}^{l} Z_{B_{i}}+Z_{E}+n Z+Z_{j-1}\right)$. If supp $E$ has at least two irreducible components, from Proposition 2.5,

$$
A_{i_{r+k}} \cdot\left(Z_{r+k-1}\right)=2 \text { and } A_{i_{j}} \cdot Z_{j-1}=1 \text { for } j \neq r+k .
$$

So

$$
A_{i_{j}} \cdot\left(\sum_{i=0}^{l} Z_{B_{i}}+Z_{E}+n Z+Z_{j-1}\right)<1 \text { for all } j \text { and all } n \text {. }
$$

Thus

$$
H^{1}\left(M, \theta\left(-\sum_{i=0}^{l} Z_{B_{i}}-Z_{E}-n Z-Z_{j-1}\right) / \theta\left(-\sum_{i=0}^{l} Z_{B_{i}}-Z_{E}-n Z-Z_{j}\right)\right)=0
$$

and the maps

$$
H^{1}\left(M, \theta\left(-\sum_{i=0}^{l} Z_{B_{i}}-Z_{E}-n Z-Z_{j}\right)\right) \rightarrow H^{1}\left(M, \theta\left(-\sum_{i=0}^{l} Z_{B_{i}}-Z_{E}-n Z-Z_{j-1}\right)\right)
$$

in (3.5) are surjective. Composing the maps, we see that

$$
\rho: H^{1}\left(M, \theta\left(-\sum_{i=0}^{l} Z_{B_{i}}-Z_{E}-n Z-Z_{j}\right)\right) \rightarrow H^{1}\left(M, \theta\left(-\sum_{i=0}^{l} Z_{B_{i}}-Z_{E}-Z_{j}\right)\right)
$$

is surjective for all $n>0$. For sufficiently large $n, \rho$ is the zero map by [7, $\$ 4$ Satz 1, p. 355]. Hence

$$
H^{1}\left[M, \vartheta\left(-\sum_{i=0}^{l} Z_{B_{i}}-Z_{E}-Z_{j}\right)\right]=0
$$




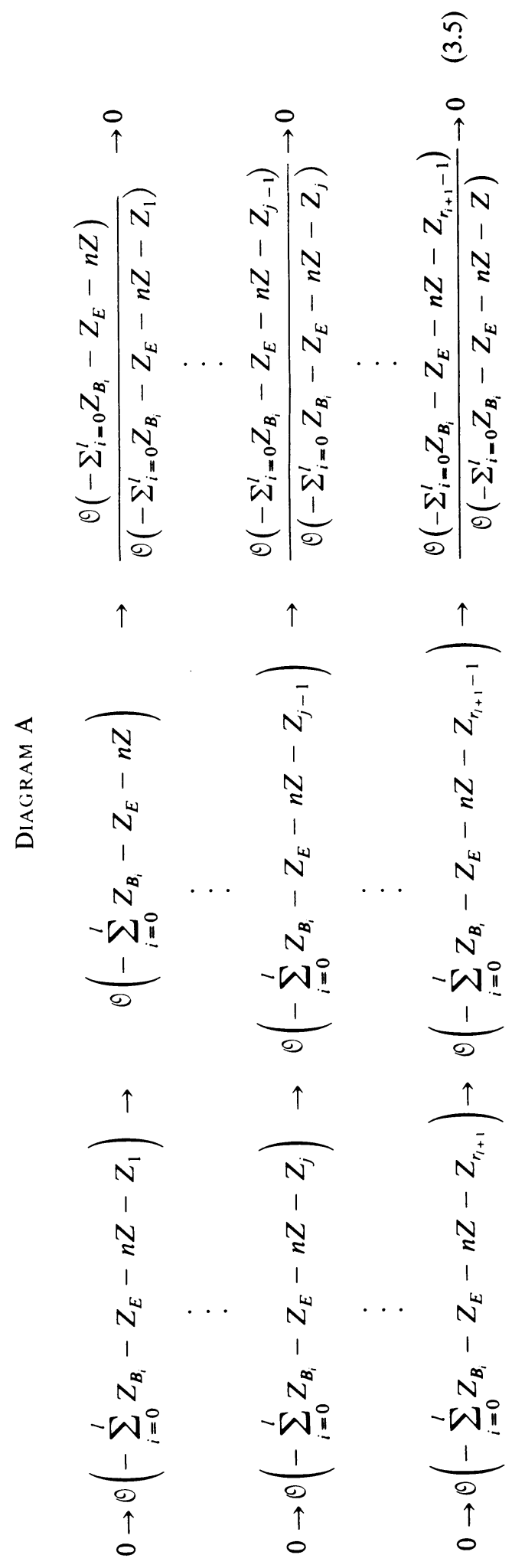


If $\operatorname{supp} E=A_{i}$ is a nonsingular elliptic curve, then $A_{i} \cdot A_{i}<-2$. By Corollary 2.6, we know that $e_{i}=z_{i}=1$, where $E=\sum e_{i} A_{i}, Z=\sum z_{i} A_{i}$. From Proposition $2.5, A_{i j} \cdot Z_{j-1}=1$ for all $j$. Therefore

$$
A_{i j} \cdot\left(\sum_{i=0}^{l} Z_{B_{i}}+Z_{E}+n Z+Z_{j-1}\right)<1 \text { for all } j \neq r+1
$$

and

$$
A_{i_{r+1}} \cdot\left(\sum_{i=0}^{l} Z_{B_{i}}+Z_{E}+n Z+Z_{r}\right)<-1 .
$$

Thus the Riemann-Roch Theorem will show that

$$
H^{1}\left\{M, \mathcal{O}\left(-\sum_{i=0}^{l} Z_{B_{i}}-Z_{E}-n Z-Z_{j-1}\right) / \mathcal{O}\left(-\sum_{i=0}^{l} Z_{B_{i}}-Z_{E}-n Z-Z_{j}\right)\right]=0
$$

for all $j$ and $n$. A similar argument will show that

$$
H^{1}\left[M, \mathcal{O}\left(-\sum_{i=0}^{l} Z_{B_{i}}-Z_{E}-Z_{j}\right)\right]=0 \text {. }
$$

In particular,

$$
H^{1}\left[M, \mathcal{O}\left(-\sum_{i=0}^{l} Z_{B_{i}}-Z_{E}-A_{1}\right)\right]=0
$$

Therefore

$$
H^{0}\left(M, \theta\left(-\sum_{i=0}^{l} Z_{B_{i}}-Z_{E}\right)\right) \rightarrow H^{0}\left(M, \theta\left(-\sum_{i=0}^{l} Z_{B_{i}}-Z_{E}\right) / \theta\left(-\sum_{i=0}^{l} Z_{B_{i}}-Z_{E}-A_{1}\right)\right)
$$

is surjective. We remark that the above argument is also applicable to the following situation. With notation as above, there exists $A_{j} \subseteq \operatorname{supp} E, A_{j} \neq$ $A_{i_{r+}}$, such that $A_{j} \cdot Z_{E}<0$.

Case (ii). Supp $E$ has at least two irreducible components and there exists $A_{i} \subseteq|E|$ such that $e_{i}=1, A_{i} \cdot Z_{E}<0$ and $A_{j} \cdot Z_{E}=0$ for all $A_{j} \subseteq|E|$ where $A_{j} \neq A_{i}$. The proof of Case (i) fails only because $A_{i_{i+k}} \neq A_{i}$, i.e., $A_{i_{++1}} \cdot Z_{E}<$ 0 . Suppose first that

$$
A_{1} \cap A_{i_{i+1}}=A_{1} \cap A_{i} \neq \varnothing .
$$

Choose a computation sequence for $Z$ with $E=Z_{k}, A_{i_{k}}=A_{i}, A_{i_{k+1}}=A_{1}$. By Proposition 2.7,

$$
H^{1}\left(M, \vartheta\left(-\sum_{i=0}^{l} Z_{B_{i}}-Z_{E}-Z_{j}\right)\right)=0 \text { for all } j
$$


Hence

$H^{0}\left(M, O\left(-\sum_{i=0}^{l} Z_{B_{i}}-Z_{E}\right)\right) \rightarrow H^{0}\left(M, \theta\left(-\sum_{i=0}^{l} Z_{B_{i}}-Z_{E}\right) / 0\left(-\sum_{i=0}^{l} Z_{B_{i}}-Z_{E}-Z_{k+1}\right)\right)$

is surjective. It follows that

$$
H^{0}\left(M, \theta\left(-\sum_{i=0}^{l} Z_{B_{i}}-Z_{E}\right)\right) \text { and } H^{0}\left(M, \frac{\theta\left(-\Sigma_{i=0}^{l} Z_{B_{i}}-Z_{E}\right)}{\theta\left(-\Sigma_{i=0}^{l} Z_{B_{i}}-Z_{E}-Z_{k+1}\right)}\right)
$$

have the same image $R$ in

$$
\begin{gathered}
H^{0}\left(M, \theta\left(-\sum_{i=0}^{l} Z_{B_{i}}-Z_{E}\right) / \theta\left(-\sum_{i=0}^{l} Z_{B_{i}}-Z_{E}-A_{1}\right)\right) . \\
0 \rightarrow H^{0}\left(M, \frac{\theta\left(-\Sigma_{i=0}^{l} Z_{B_{i}}-Z_{E}-Z_{k}\right)}{\theta\left(-\Sigma_{i=0}^{l} Z_{B_{i}}-Z_{E}-Z_{k+1}\right)}\right) \rightarrow H^{0}\left(M, \frac{\theta\left(-\Sigma_{i=0}^{l} Z_{B_{i}}-Z_{E}\right)}{\theta\left(-\Sigma_{i=0}^{l} Z_{B_{i}}-Z_{E}-Z_{k+1}\right)}\right) \\
\rightarrow H^{0}\left(M, \frac{\theta\left(-\Sigma_{i=0}^{l} Z_{B_{i}}-Z_{E}\right)}{\theta\left(-\Sigma_{i=0}^{l} Z_{B_{i}}-Z_{E}-Z_{k}\right)}\right) \rightarrow 0
\end{gathered}
$$

is an exact sequence. Thus the image of

$$
H^{0}\left(M, \vartheta\left(-\sum_{i=0}^{l} Z_{B_{i}}-Z_{E}-Z_{k}\right) / \theta\left(-\sum_{i=0}^{l} Z_{B_{i}}-Z_{E}-Z_{k+1}\right)\right]
$$

which is injected into

$$
H^{0}\left[M, \theta\left(-\sum_{i=0}^{l} Z_{B_{i}}-Z_{E}\right) / \theta\left(-\sum_{i=0}^{l} Z_{B_{i}}-Z_{E}-A_{1}\right)\right]
$$

via the natural map is contained in $R$. If

$$
H^{0}\left[M, \vartheta\left(-\sum_{i=0}^{l} Z_{B_{i}}-Z_{E}-Z_{k}\right) / \vartheta\left(-\sum_{i=0}^{l} Z_{B_{i}}-Z_{E}-Z_{k+1}\right)\right] \neq 0
$$

then the elements of $R$ have no common zeros on $A_{1}-\left(A_{1} \cap A_{i}\right)$ as sections of the line bundle $L$ on $A_{1}$ associated to $\theta\left(-\sum_{i=0}^{l} Z_{B_{i}}-Z_{E}\right) / \theta\left(-\sum_{i=0}^{l} Z_{B_{i}}-\right.$ $\left.Z_{E}-A_{1}\right)$. If

$$
H^{0}\left[M, \vartheta\left(-\sum_{i=0}^{l} Z_{B_{i}}-Z_{E}-Z_{k}\right) / \theta\left(-\sum_{i=0}^{l} Z_{B_{i}}-Z_{E}-Z_{k+1}\right)\right]=0
$$

then $A_{1} \cdot\left(\sum_{i=0}^{l} Z_{B_{i}}+Z_{E}\right)=0$. Hence

$$
H^{0}\left[M, \mathcal{O}\left(-\sum_{i=0}^{l} Z_{B_{i}}-Z_{E}\right) / \mathcal{O}\left(-\sum_{i=0}^{l} Z_{B_{i}}-Z_{E}-A_{1}\right)\right]=\mathbf{C}
$$


We claim that

$$
H^{0}\left(M, \theta\left(-\sum_{i=0}^{l} Z_{B_{i}}-Z_{E}\right)\right) \rightarrow H^{0}\left(M, \theta\left(-\sum_{i=0}^{l} Z_{B_{i}}-Z_{E}\right) / 0\left(-\sum_{i=0}^{l} Z_{B_{i}}-Z_{E}-A_{1}\right)\right)
$$

is surjective. It suffices to prove that the map is not a zero map. Suppose $\pi$ is the minimal resolution; then $Z_{E}=E$ and hence $A_{i} \cdot Z_{E}<-2$. So

$$
A_{i} \cdot\left(\sum_{i=0}^{l} Z_{B_{i}}+Z_{E}\right)<-2
$$

and

$$
H^{0}\left[M, \vartheta\left(-\sum_{i=0}^{l} Z_{B_{i}}-Z_{E}\right) / \mathcal{O}\left(-\sum_{i=0}^{l} Z_{B_{i}}-Z_{E}-A_{i}\right)\right]>3 .
$$

The image of

$$
\begin{aligned}
& \rho: H^{0}\left(M, \vartheta\left(-\sum_{i=0}^{l} Z_{B_{i}}-Z_{E}\right)\right) \\
& \rightarrow H^{0}\left(M, \theta\left(-\sum_{i=0}^{l} Z_{B_{i}}-Z_{E}\right) / \theta\left(-\sum_{i=0}^{l} Z_{B_{i}}-Z_{E}-A_{i}\right)\right)
\end{aligned}
$$

is a subspace $S$ of codimension 1 in

$$
H^{0}\left[M, \vartheta\left(-\sum_{i=0}^{l} Z_{B_{i}}-Z_{E}\right) / \mathcal{O}\left(-\sum_{i=0}^{l} Z_{B_{i}}-Z_{E}-A_{i}\right)\right] \text {. }
$$

Hence the elements of $S$ have no common zeros as sections of the line bundle $L_{i}$ on $A_{i}$ associated to

$$
\vartheta\left(-\sum_{i=0}^{l} Z_{B_{i}}-Z_{E}\right) / \theta\left(-\sum_{i=0}^{l} Z_{B_{i}}-Z_{E}-A_{i}\right)
$$

by Proposition 2.8. If $\pi$ is not the minimal resolution, we still get that the elements of $S$ have no common zeros as sections of the line bundle $L_{i}$ on $A_{i}$ associated to

$$
\vartheta\left(-\sum_{i=0}^{l} Z_{B_{i}}-Z_{E}\right) / \theta\left(-\sum_{i=0}^{l} Z_{B_{i}}-Z_{E}-A_{i}\right)
$$

by an easy case-by-case check using Proposition 2.8 with $Y=\sum_{i=0}^{l} Z_{B_{i}}+Z_{E}$. It follows that

$$
H^{0}\left(M, \theta\left(-\sum_{i=0}^{l} Z_{B_{i}}-Z_{E}\right)\right) \rightarrow H^{0}\left(M, \theta\left(-\sum_{i=0}^{l} Z_{B_{i}}-Z_{E}\right) / \theta\left(-\sum_{i=0}^{l} Z_{B_{i}}-Z_{E}-A_{1}\right)\right)
$$

is not a zero map and, hence, a surjective map.

To finish the proof of Case (ii), it remains to consider those $A_{1} \ell|E|$ such that $A_{1} \cap A_{i}=\varnothing$ and the computation sequence for $Z$ starting from $A_{1}$ in order to reach $|E|$ must first reach $A_{i}$. Choose a computation sequence for $Z$ 
with $E=Z_{k}, A_{i_{k}}=A_{i}, A_{i_{k+1}} \cap A_{i} \neq \varnothing, A_{i_{k+t}}=A_{1}, A_{1} \underline{Z}\left|Z_{k+t-1}\right|$ and $A_{i j}$, $k+1<j<k+t$, are distinct to each other such that $A_{i,} \subseteq|E|$. By Proposition 2.7,

$$
H^{1}\left[M, \mathcal{O}\left(-\sum_{i=0}^{l} Z_{B_{i}}-Z_{E}-Z_{j}\right)\right]=0 \text { for all } j
$$

Hence

$H^{0}\left(M, O\left(-\sum_{i=0}^{l} Z_{B_{i}}-Z_{E}\right)\right) \rightarrow H^{0}\left(M, O\left(-\sum_{i=0}^{l} Z_{B_{i}}-Z_{E}\right) / O\left(-\sum_{i=0}^{l} Z_{B_{i}}-Z_{E}-Z_{k+t}\right)\right)$

is surjective. It follows that

$$
H^{0}\left(M, O\left(-\sum_{i=0}^{l} Z_{B_{i}}-Z_{E}\right)\right) \text { and } H^{0}\left(M, \frac{\theta\left(-\Sigma_{i-0}^{l} Z_{B_{i}}-Z_{E}\right)}{O\left(-\Sigma_{i=0}^{l} Z_{B_{i}}-Z_{E}-Z_{k+t}\right)}\right)
$$

have the same image $R$ in

$$
\begin{gathered}
H^{0}\left(M, \theta\left(-\sum_{i=0}^{l} Z_{B_{i}}-Z_{E}\right) / \theta\left(-\sum_{i=0}^{l} Z_{B_{i}}-Z_{E}-A_{1}\right)\right) . \\
0 \rightarrow H^{0}\left(M, \frac{\theta\left(-\Sigma_{i=0}^{l} Z_{B_{i}}-Z_{E}-Z_{k+t-1}\right)}{\theta\left(-\Sigma_{i=0}^{l} Z_{B_{i}}-Z_{E}-Z_{k+t}\right)}\right) \rightarrow H^{0}\left(M, \frac{\theta\left(-\Sigma_{i=0}^{l} Z_{B_{i}}-Z_{E}\right)}{\theta\left(-\sum_{i=0}^{l} Z_{B_{i}}-Z_{E}-Z_{k+\imath}\right)}\right) \\
\rightarrow H^{0}\left(M, \frac{\theta\left(-\Sigma_{i=0}^{l} Z_{B_{i}}-Z_{E}\right)}{\theta\left(-\Sigma_{i=0}^{l} Z_{B_{i}}-Z_{k+t-1}\right)}\right) \rightarrow 0
\end{gathered}
$$

is an exact sequence. Thus the image of

$$
H^{0}\left[M, \mathcal{O}\left(-\sum_{i=0}^{l} Z_{B_{i}}-Z_{E}-Z_{k+t-1}\right) / \mathcal{O}\left(-\sum_{i=0}^{l} Z_{B_{i}}-Z_{E}-Z_{k+t}\right)\right]
$$

which is injected into

$$
H^{0}\left[M, \mathcal{O}\left(-\sum_{i=0}^{l} Z_{B_{i}}-Z_{E}\right) / \mathcal{O}\left(-\sum_{i=0}^{l} Z_{B_{i}}-Z_{E}-A_{1}\right)\right]
$$

via the natural map is contained in $R$. If

$$
H^{0}\left[M, \theta\left(-\sum_{i=0}^{l} Z_{B_{i}}-Z_{E}-Z_{k+t-1}\right) / \mathcal{O}\left(-\sum_{i=0}^{l} Z_{B_{i}}-Z_{E}-Z_{k+t}\right)\right] \neq 0,
$$

then the elements of $R$ have no common zeros on $A_{1}-\left(A_{1} \cap A_{i_{k+1-1}}\right)$ as sections of the line bundle $L_{1}$ on $A_{1}$ associated to

$$
\Theta\left(-\sum_{i=0}^{l} Z_{B_{i}}-Z_{E}\right) / \mathcal{O}\left(-\sum_{i=0}^{l} Z_{B_{i}}-Z_{E}-A_{1}\right) \text {. }
$$


If

$$
H^{0}\left[M, \vartheta\left(-\sum_{i=0}^{l} Z_{B_{i}}-Z_{E}-Z_{k+t-1}\right) / \vartheta\left(-\sum_{i=0}^{l} Z_{B_{i}}-Z_{E}-Z_{k+t}\right)\right]=0 \text {, }
$$

then $A_{1} \cdot\left(\sum_{i=0}^{l} Z_{B_{i}}+Z_{E}\right)=0$. Hence

$$
H^{0}\left[M, \vartheta\left(-\sum_{i=0}^{l} Z_{B_{i}}-Z_{E}\right) / \mathcal{O}\left(-\sum_{i=0}^{l} Z_{B_{i}}-Z_{E}-A_{1}\right)\right]=\mathbf{C} .
$$

But by induction, we know that the elements of the image of

$$
\begin{aligned}
& H^{0}\left(M, O\left(-\sum_{i=0}^{l} Z_{B_{i}}-Z_{E}\right)\right) \\
& \rightarrow H^{0}\left(M, O\left(-\sum_{i=0}^{l} Z_{B_{i}}-Z_{E}\right) / O\left(-\sum_{i=0}^{l} Z_{B_{i}}-Z_{E}-A_{i_{k+1-1}}\right)\right)
\end{aligned}
$$

have no common zeros on $A_{i_{k+t-1}}-\left(A_{i_{k+t-1}} \cap A_{i_{k+t-2}}\right)$ as sections of the line bundle $L_{i_{k+t-1}}$ on $A_{i_{k+t-1}}$ associated to

$$
\theta\left(-\sum_{i=0}^{l} Z_{B_{i}}-Z_{E}\right) / \theta\left(-\sum_{i=0}^{l} Z_{B_{i}}-Z_{E}-A_{i_{k+t-1}}\right) \text {. }
$$

It follows that

$$
H^{0}\left(M, \theta\left(-\sum_{i=0}^{l} Z_{B_{i}}-Z_{E}\right)\right) \rightarrow H^{0}\left(M, \theta\left(-\sum_{i=0}^{l} Z_{B_{i}}-Z_{E}\right) / \theta\left(-\sum_{i=0}^{l} Z_{B_{i}}-Z_{E}-A_{1}\right)\right)
$$

is again surjective. So far we have proved $\theta(-Z) \subseteq m \theta$. But $m \theta \subseteq \theta(-Z)$ by [2]. This completes our proof of the theorem.

Proposition 3.13. Let $\pi: M \rightarrow V$ be the minimal good resolution of a normal two-dimensional Stein space with $p$ as its only maximally elliptic singularity. Let

$$
Z_{B_{0}}=Z, Z_{B_{1}}, \ldots, Z_{B_{l}}, Z_{E}=Z_{B_{l+1}}
$$

be the elliptic sequence. Then for any $0<h<l$, there exists $f \in$ $H^{0}\left(M, \mathcal{O}\left(-\sum_{i=0}^{h} Z_{B_{i}}\right)\right)$ such that $f \notin H^{0}\left(M, \mathcal{O}\left(-\sum_{i=0}^{h+1} Z_{B_{i}}\right)\right)$. In fact the vanishing order of $f$ on $A_{j}$ is precisely $\sum_{i=0}^{h} B_{i} z_{j}$, where $Z_{B_{i}}=\sum_{k B_{i}} z_{k} A_{k}$ and $A_{j} \subseteq B_{h+1}$.

Proof. By the definition of maximally elliptic singularity, $\operatorname{dim} H^{1}(M, \theta)$ $=$ the length of the elliptic sequence. By the proof of Theorem 3.9, we know that maximal ellipticity implies

$$
H^{1}\left(M, \theta_{\sum_{i=0}^{h} z_{B_{i}}}\right)=\mathbf{C}^{h+1} \text { for all } 0<h<l .
$$

Moreover,

$$
H^{0}\left(M, \vartheta_{\sum_{i=0}^{h+1} z_{B_{i}}}\right) \rightarrow H^{0}\left(M, \Theta_{\sum_{i=0}^{h} z_{B_{i}}}\right)
$$

are surjective. Consider the following commutative diagram with exact rows:

Diagram B (page 305). 


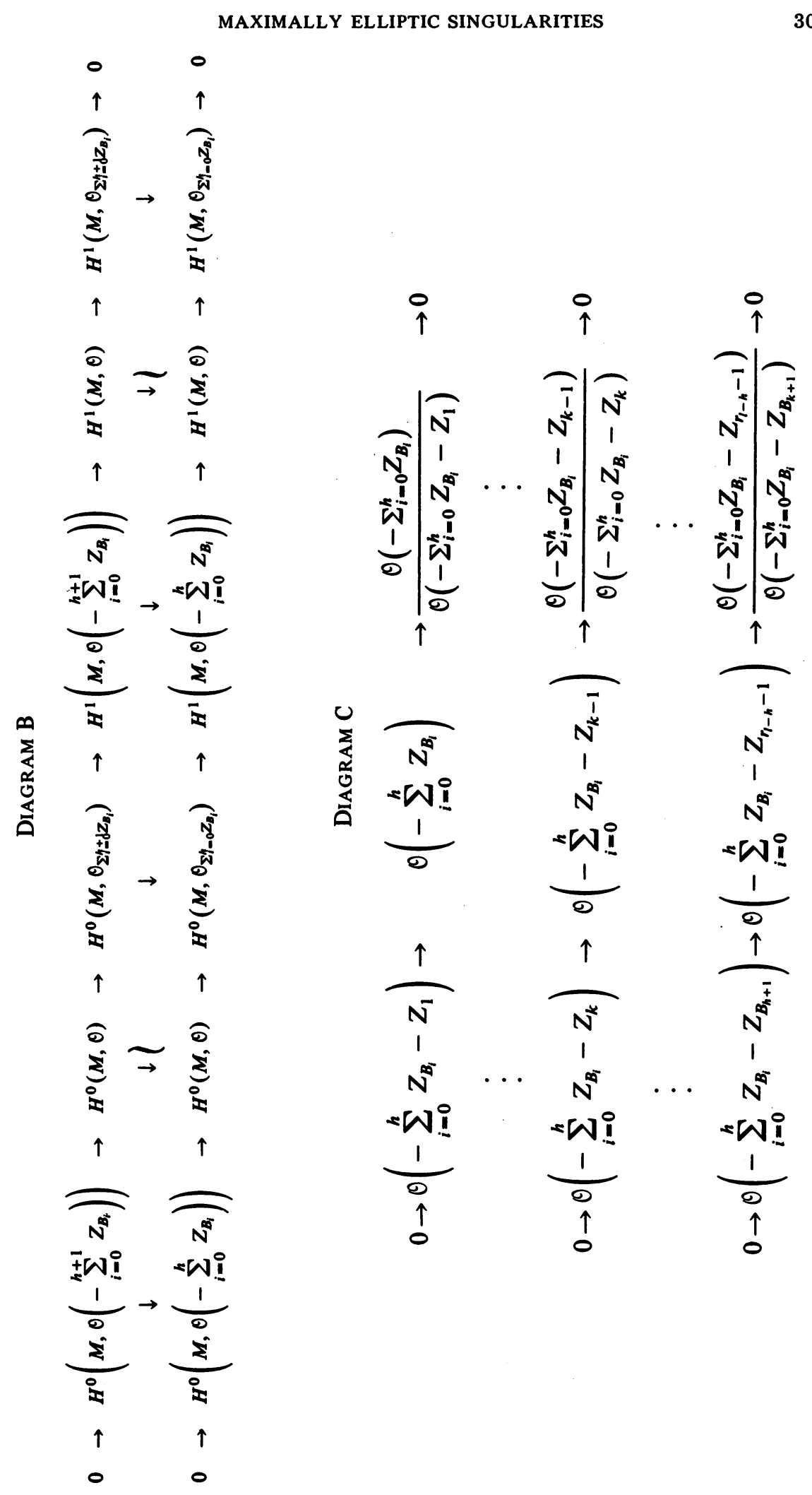


Since $H^{1}\left(M, \theta\left(-\sum_{i=0}^{l} Z_{B_{i}}-Z_{E}\right)\right)=0$ by Proposition 2.8,

$$
H^{0}(M, \theta) \rightarrow H^{0}\left(M, \theta_{\Sigma_{i=0}^{\prime} z_{B_{i}}}+z_{E}\right)
$$

is surjective. It follows that

$$
H^{0}(M, \theta) \rightarrow H^{0}\left(M, \theta_{\Sigma_{i=0}^{n} z_{B_{i}}}\right)
$$

are surjective for all $0 \leqslant h \leqslant l$. An easy diagram chase will show that there exists $f \in H^{0}\left(M, \theta\left(-\sum_{i=0}^{h} Z_{B_{i}}\right)\right)$ but $f \notin H^{0}\left(M, \theta\left(-\sum_{i=0}^{h+1} Z_{B_{i}}\right)\right)$. Let $A_{j} \subseteq$ $B_{h+1}$. Choose a computation sequence for $Z_{B_{h+1}}$ of the following form:

$$
Z_{0}=0, Z_{1}=A_{j}, \ldots, Z_{r_{l-h}}=Z_{B_{h+1}} \text {. }
$$

Look at the sheaf exact sequence:

Diagram C (page 305).

If the vanishing order of $f$ on $A_{j}$ is larger than $\sum_{i=0}^{l} B_{i} z_{j}$, then the usual cohomology exact sequence argument will show that $f \in$ $H^{0}\left(M, \mathcal{O}\left(-\sum_{i=0}^{h+1} Z_{B_{i}}\right)\right)$, which is a contradiction. Q.E.D.

The following corollary is a partial converse of Theorem 3.2.

COROLlary 3.14. Let $V$ be a normal two-dimensional Stein space with $p$ as its only maximally elliptic singular point. Let

$$
Z_{B_{0}}=Z, Z_{B_{1}}, \ldots, Z_{B_{1}}, Z_{B_{l+1}}=Z_{E}
$$

be the elliptic sequence. If there exists $A_{1} \subseteq|E|$ such that the coefficients of $A_{1}$ in $Z_{B_{i}}, 0<i<l$, are equal, then there exist $f \in H^{0}(M, \theta), \omega \in H^{0}(M-$ $A, \Omega)$ such that $\omega, f \omega, \ldots, f^{l+1} \omega$ forms a basis of $H_{*}^{1}(M, \theta)$.

Proof. An easy consequence of Theorems 3.7, 3.11 and Proposition 3.13. The following theorem will be useful in calculating the Hilbert function $\operatorname{dim} m^{n} / m^{n+1}$.

THEOREM 3.15. Let $\pi: M \rightarrow V$ be the minimal good resolution of a normal 2-dimensional Stein space with $p$ as its only maximally elliptic singularity. If $Z_{E} \cdot Z_{E}<-3$, then

$$
H^{0}(M, \theta(-Z)) \otimes_{\mathrm{C}} H^{0}(M, \theta(-n Z)) \rightarrow H^{0}(M, \theta(-(n+1) Z))
$$

is surjective for all $n>1$. If we assume further that the length of the elliptic sequence is equal to two, then the above map is surjective for all $n>1$. In this case,

$$
m^{n} \simeq H^{0}(A, \Theta(-n Z)) \text { for all } n>0
$$

where $A=\pi^{-1}(p)$.

Proof. It is true that $H^{0}(A, \theta(-Z))=\operatorname{proj} \lim H^{0}(U, \theta(-Z)), U$ a neighborhood of $A$. Since $Z$ is minimal, $H^{0}(A, \theta(-Z))=m$. By Theorem 3.7 , the elliptic sequence is of the form

$$
Z_{B_{0}}=Z, Z_{B_{1}}, \ldots, Z_{B_{l}}, Z_{E} \text { and } K^{\prime}=-\sum_{i=0}^{l} Z_{B_{i}}-E \text {. }
$$


Step 1. We are going to show

$$
\begin{aligned}
H^{0}\left[M, \theta\left(-\sum_{i=0}^{l} Z_{B_{i}}-Z_{E}\right)\right] & \otimes_{C} H^{0}\left[M, \theta\left(-n Z-\sum_{i=1}^{l} Z_{B_{i}}-Z_{E}\right)\right] \\
& \rightarrow H^{0}\left(M, \theta\left(-(n+1) Z-2 \sum_{i=1}^{l} Z_{B_{i}}-2 Z_{E}\right)\right]
\end{aligned}
$$

is surjective for all $n>1$. It suffices to show

$$
\begin{gathered}
\tau: H^{0}\left(M, \frac{\theta\left(-\Sigma_{i=0}^{l} Z_{B_{i}}-Z_{E}\right)}{\theta\left(-2 Z-\Sigma_{i=1}^{l} Z_{B_{i}}-Z_{E}\right)}\right) \otimes_{C} H^{0}\left(M, \frac{\theta\left(-n Z-\Sigma_{i=1}^{l} Z_{B_{t}}-Z_{E}\right)}{\theta\left(-(n+1) Z-\Sigma_{i-1}^{l} Z_{B_{i}}-Z_{E}\right)}\right) \\
\rightarrow H^{0}\left(M, \frac{\theta\left(-(n+1) Z-2 \Sigma_{i=1}^{l} Z_{B_{i}}-2 Z_{E}\right)}{\theta\left(-(n+2) Z-2 \Sigma_{i=1}^{l} Z_{B_{i}}-2 Z_{E}\right)}\right)
\end{gathered}
$$

is surjective for all $n \geqslant 1$.

Let us first demonstrate this fact. We first show that the image of

$$
H^{0}\left[M, \vartheta\left(-\sum_{i=0}^{l} Z_{B_{i}}-Z_{E}\right)\right] \otimes_{\mathrm{C}} H^{0}\left[M, \vartheta\left(-n Z-\sum_{i=1}^{l} Z_{B_{i}}-Z_{E}\right)\right]
$$

contains

$$
H^{0}\left\{M, \vartheta\left(-m Z-2 \sum_{i=1}^{l} Z_{B_{i}}-2 Z_{E}\right)\right\}
$$

for some $m$. Let

$$
f_{1}, \ldots, f_{s} \in H^{0}\left[M, \vartheta\left(-n Z-\sum_{i=1}^{l} Z_{B_{i}}-Z_{E}\right)\right]
$$

generate $\theta\left(-n Z-\sum_{i=1}^{l} Z_{B_{i}}-Z_{E}\right)$ as an $\theta$-module. Proposition 2.8 and the proof of Theorem 3.12 guarantee that such $f_{i}$ 's do exist. The $\theta$-module map

$$
\rho: \bigoplus_{S} \mathcal{O}\left(-Z-\sum_{i=1}^{l} Z_{B_{i}}-Z_{E}\right) \rightarrow \mathcal{\Theta}\left(-(n+1) Z-2 \sum_{i=1}^{l} Z_{B_{i}}-2 Z_{E}\right)
$$

given by $\left(g_{1}, \ldots, g_{s}\right) \rightarrow \sum f_{i} g_{i}$ is then surjective. Let $K=\operatorname{ker} \rho$.

$$
0 \rightarrow K \rightarrow \bigoplus_{s} \Theta\left(-Z-\sum_{i=1}^{l} Z_{B_{i}}-Z_{E}\right) \stackrel{\rho}{\rightarrow} \vartheta\left(-(n+1) Z-2 \sum_{i=1}^{l} Z_{B_{i}}-2 Z_{E}\right) \rightarrow 0
$$

is exact. Multiplying by $\theta(-k Z)$, we get

Diagram D (page 308).

with the vertical maps the inclusion maps, is commutative. The verification that the first line is exact is the same as the verification that $[19,(5.5)]$ was exact. 

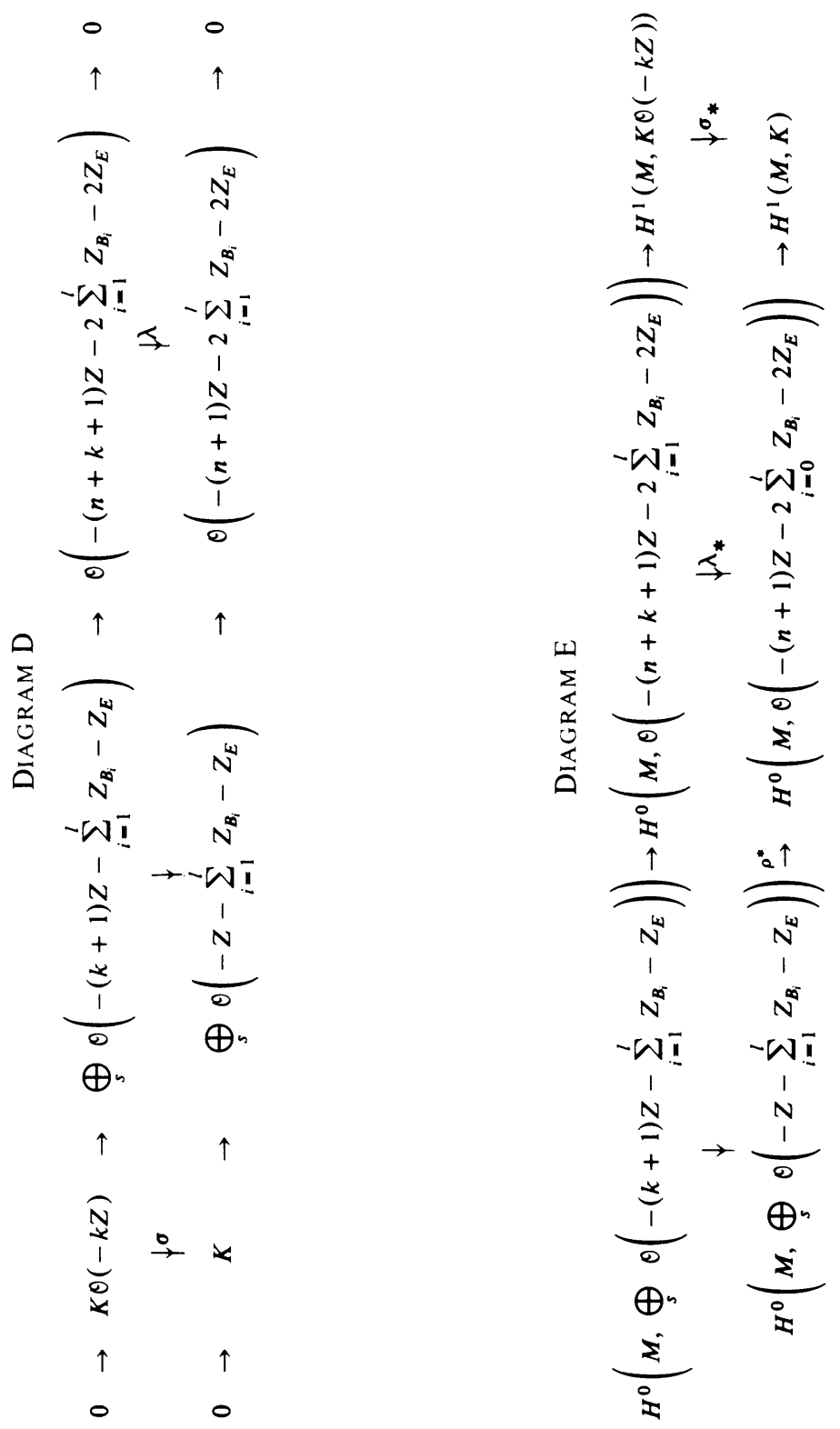


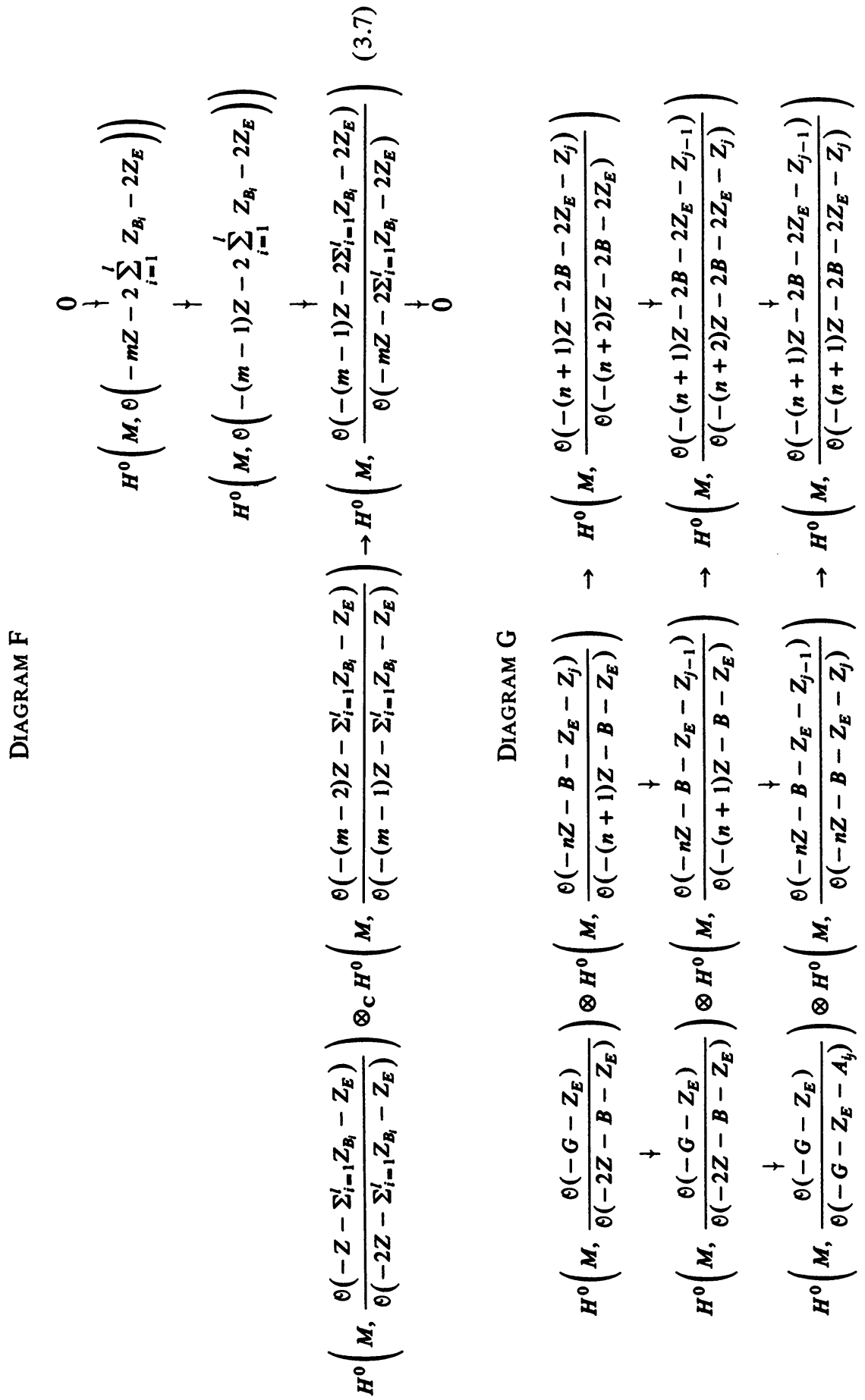


is commutative with exact rows. By [7, $\$ 4$ Satz 1, p. 355], $\sigma_{*}$ is the zero map for sufficiently large $k$. Then given

$$
h \in H^{0}\left[M, \theta\left(-(n+k+1) Z-2 \sum_{i=1}^{l} Z_{B_{i}}-2 Z_{E}\right)\right],
$$

$\lambda_{*}(h)=\rho_{*}(g)$ for some $g$, by exactness. Letting $m=n+k+1$, we have that the image of

$$
H^{0}\left[M, \vartheta\left(-Z-\sum_{i=1}^{l} Z_{B_{i}}-Z_{E}\right)\right] \otimes_{\mathrm{C}} H^{0}\left[M, \theta\left(-n Z-\sum_{i=1}^{l} Z_{B_{i}}-Z_{E}\right)\right]
$$

contains

$$
H^{0}\left[M, \vartheta\left(-m Z-2 \sum_{i=1}^{l} Z_{B_{i}}-2 Z_{E}\right)\right]
$$

as required.

If $m>n+1>2$, we shall show that the image of

$$
H^{0}\left[M, \vartheta\left(-\sum_{i=0}^{l} Z_{B_{i}}-Z_{E}\right)\right] \otimes_{\mathrm{C}} H^{0}\left[M, \vartheta\left(-n Z-\sum_{i=1}^{l} Z_{B_{i}}-Z_{E}\right)\right]
$$

contains

$$
H^{0}\left[M, \theta\left(-(m-1) Z-2 \sum_{i=1}^{l} Z_{B_{i}}-2 Z_{E}\right)\right] \text {. }
$$

By induction argument, we will be done. Look at the diagram:

$$
\text { Diagram F (page 309). }
$$

Since $m>2$,

$$
H^{1}\left[M, \vartheta\left(-m Z-2 \sum_{i=1}^{l} Z_{B_{i}}-2 Z_{E}\right)\right]=0
$$

by Proposition 2.7. Hence the vertical sequence is exact. We also notice that the maps

$$
\begin{array}{r}
H^{0}\left[M, \theta\left(-Z-\sum_{i=1}^{l} Z_{B_{i}}-Z_{E}\right)\right] \rightarrow H^{0}\left[M, \frac{\theta\left(-Z-\sum_{i=1}^{l} Z_{B_{i}}-Z_{E}\right)}{\theta\left(-2 Z-\sum_{i=1}^{l} Z_{B_{i}}-Z_{E}\right)}\right], \\
H^{0}\left[M, \vartheta\left(-(m-2) Z-\sum_{i=1}^{l} Z_{B_{i}}-Z_{E}\right)\right] \\
\rightarrow H^{0}\left[M, \frac{\theta\left(-(m-2) Z-\sum_{i=1}^{l} Z_{B_{i}}-Z_{E}\right)}{\theta\left(-(m-1) Z-\sum_{i=1}^{l} Z_{B_{i}}-Z_{E}\right)}\right]
\end{array}
$$


are surjective because

$$
\begin{aligned}
H^{1}[M, \vartheta(-2 Z & \left.\left.-\sum_{i=1}^{l} Z_{B_{i}}-Z_{E}\right)\right]=0 \\
& =H^{1}\left(M, \vartheta\left(-(m-1) Z-\sum_{i=1}^{l} Z_{B_{i}}-Z_{E}\right)\right)
\end{aligned}
$$

by Proposition 2.7. The horizontal map in (3.7) is surjective by hypothesis. It follows easily that the image of

$$
H^{0}\left[M, \vartheta\left(-Z-\sum_{i=1}^{l} Z_{B_{i}}-Z_{E}\right)\right] \otimes H^{0}\left[M, \vartheta\left(-n Z-\sum_{i=1}^{l} Z_{B_{i}}-Z_{E}\right)\right]
$$

contains

$$
H^{0}\left[M, \vartheta\left(-(m-1) Z-2 \sum_{i=1}^{l} Z_{B_{i}}-2 Z_{E}\right)\right] .
$$

It remains to prove (3.6) is surjective for all $n$. The proof breaks up into three subcases.

(i) There is an $A_{i}$, call it $A_{1}$, such that $Z_{E} \cdot Z_{E}+1<A_{1} \cdot Z_{E}<-2$.

(ii) There is an $A_{i}$, call it $A_{1}$, such that $A_{1} \cdot Z_{E}=Z_{E} \cdot Z_{E}$.

(iii) $A_{i} \cdot Z_{E}=-1$ or 0 , all $A_{i} \subseteq \operatorname{supp} E$. Take $A_{1} \cdot Z_{E}=-1$.

In case (i), all irreducible components are nonsingular rational curves. Choose a computation sequence for $Z$ with $E=Z_{k}, Z_{r_{1+1}}=Z$ and $A_{i_{k}}=A_{1}$. Consider

$$
\begin{gathered}
\tau_{j}: H^{0}\left(M, \frac{\theta\left(-Z-B-Z_{E}\right)}{\theta\left(-Z-B-Z_{E}-A_{j}\right)}\right) \otimes H^{0}\left(M, \frac{\theta\left(-n Z-B-Z_{E}-Z_{j-1}\right)}{\theta\left(-n Z-B-Z_{E}-Z_{j}\right)}\right) \\
\rightarrow H^{0}\left(M, \frac{\theta\left(-(n+1) Z-2 B-2 Z_{E}-Z_{j-1}\right.}{\theta\left(-(n+1) Z-2 B-2 Z_{E}-Z_{j}\right)}\right)
\end{gathered}
$$

where $B=\sum_{i=1}^{l} Z_{B_{i}}$

To show that $\tau$ in (3.6) in surjective, it will suffice to show that $\tau_{j}$ in (3.8) is surjective for all $j$. Indeed, since all of the first cohomology groups

$$
H^{1}\left[M, \vartheta\left(-n Z-\sum_{i=1}^{l} Z_{B_{i}}-Z_{E}-Z_{j}\right)\right]=0 \text { for all } j
$$

by Proposition 2.7,

$$
H^{0}\left[M, \vartheta\left(-n Z-\sum_{i=1}^{l} Z_{B_{i}}-Z_{E}\right) / \theta\left(-(n+1) Z-\sum_{i=1}^{l} Z_{B_{i}}-Z_{E}\right)\right]
$$


can be written via successive quotients as

$$
\begin{aligned}
0 & \rightarrow H^{0}\left[M, \frac{\theta\left(-n Z-B-Z_{E}-Z_{j}\right)}{\theta\left(-(n+1) Z-B-Z_{E}\right)}\right] \\
& \rightarrow H^{0}\left[M, \frac{\theta\left(-n Z-B-Z_{E}-Z_{j-1}\right)}{\theta\left(-(n+1) Z-B-Z_{E}\right)}\right] \\
& \rightarrow H^{0}\left[M, \frac{\theta\left(-n Z-B-Z_{E}-Z_{j-1}\right)}{\theta\left(-n Z-B-Z_{E}-Z_{j}\right)}\right] \rightarrow 0, \quad 1<j<r_{l+1}-1,
\end{aligned}
$$

where we denote $B=\sum_{i=1}^{l} Z_{B_{i}}$.

$$
H^{0}\left(M, O\left(-(n+1) Z-2 \sum_{i=1}^{l} Z_{B_{i}}-2 Z_{E}\right) / O\left(-(n+2) Z-2 \sum_{i=1}^{l} Z_{B_{i}}-2 Z_{E}\right)\right)
$$

also can be written via similar successive quotients. Moreover,

$$
H^{1}\left[M, \theta\left(-\sum_{i=0}^{l} Z_{B_{i}}-Z_{E}-A_{i_{j}}\right)\right]=0
$$

by Proposition 2.8 and the proof of Theorem 3.12. Now consider the commutative diagrams:

Diagram G (page 309),

$1<j<r_{l+1}$, where we denote $\sum_{i=0}^{l} Z_{B_{i}}=G$ and $\Sigma_{i=1}^{l} Z_{B_{i}}=B$. Thus if (3.8) is surjective for all $j,(3.6)$ is also surjective.

Suppose that the target space in (3.8) is nonzero, i.e.,

$$
-A_{i_{j}} \cdot\left((n+1) Z+2 \sum_{i=1}^{l} Z_{B_{i}}+2 Z_{E}+Z_{j-1}\right)>0 \text {. }
$$

We need

$$
-A_{i_{j}} \cdot\left(Z+\sum_{i=1}^{l} Z_{B_{i}}+Z_{E}\right)>0
$$

and

$$
-A_{i_{j}} \cdot\left(n Z+\sum_{i=1}^{l} Z_{B_{i}}+Z_{E}+Z_{j-1}\right)>0
$$

For $j \neq k, A_{i j} \cdot Z_{j-1}=1$. If $-A_{i j} \cdot\left(\sum_{i=0}^{l} Z_{B_{i}}+Z_{E}\right)>0$, then

$$
-A_{i_{j}}\left(n Z+\sum_{i=1}^{l} Z_{B_{i}}+Z_{E}+Z_{j-1}\right)>0
$$


If $-A_{i_{j}} \cdot\left(\sum_{i=0}^{l} Z_{B_{i}}+Z_{E}\right)=0$, then

$$
\begin{aligned}
-A_{i_{j}}(n Z & \left.+\sum_{i=1}^{l} Z_{B_{i}}+Z_{E}+Z_{j-1}\right) \\
& =-A_{i_{j}} \cdot\left((n+1) Z+2 \sum_{i=1}^{l} Z_{B_{i}}+2 Z_{E}+Z_{j-1}\right)>0 .
\end{aligned}
$$

For $j=k, A_{i_{k}} \cdot Z_{k-1}=2$. By construction $A_{i_{k}} \cdot Z_{E}<-2$ and so (3.8) is surjective for all $j$.

Let us now do case (ii). Suppose supp $E$ has more than one irreducible component. The proof of case (i) fails only because the maps

$$
\begin{aligned}
H^{0}\left[M, \theta\left(-\sum_{i=0}^{l} Z_{B_{i}}-Z_{E}\right)\right] \\
\\
\quad \rightarrow H^{0}\left[M, \theta\left(-\sum_{i=0}^{l} Z_{B_{i}}-Z_{E}\right) / \theta\left(-\sum_{i=0}^{l} Z_{B_{i}}-Z_{E}-A_{i_{k}}\right)\right]
\end{aligned}
$$

and

$$
\begin{aligned}
H^{0}\left[M, \vartheta\left(-\sum_{i=0}^{l} Z_{B_{i}}-Z_{E}\right)\right] \\
\\
\rightarrow H^{0}\left[M, \vartheta\left(-\sum_{i=0}^{l} Z_{B_{i}}-Z_{E}\right) / \theta\left(-\sum_{i=0}^{l} Z_{B_{i}}-Z_{E}-A_{i_{i+k}}\right)\right]
\end{aligned}
$$

need not be surjective, where $A_{i_{+k}} \&|E|$ and the computation sequence starting from $A_{i_{t+k}}$ in order to reach $|E|$ must first reach $A_{1}$. In (3.8),

$$
H^{0}\left(M, \frac{\theta\left(-\Sigma_{i=0}^{l} Z_{B_{i}}-Z_{E}\right)}{\theta\left(-\Sigma_{i=0}^{l} Z_{B_{i}}-Z_{E}-A_{i_{k}}\right)}\right)=H^{0}\left(M, \frac{\theta\left(-\Sigma_{i=0}^{l} Z_{B_{i}}-Z_{E}\right)}{\theta\left(-\Sigma_{i=0}^{l} Z_{B_{i}}-Z_{E}-A_{1}\right)}\right)
$$

must be replaced by the subspace $S$ of Proposition 2.8 .

$$
\operatorname{dim} S=-A_{1}\left(\sum_{i=0}^{l} Z_{B_{i}}+Z_{E}\right)=-A_{1} \cdot Z_{E}=-Z_{E} \cdot Z_{E}>2
$$

Also,

$$
\begin{aligned}
\operatorname{dim} H^{0}(M, O(-n Z & \left.\left.-\sum_{i=1}^{l} Z_{B_{i}}-Z_{E}-Z_{k-1}\right) / O\left(-n Z-\sum_{i=1}^{l} Z_{B_{i}}-Z_{E}-Z_{k}\right)\right) \\
& =-A_{1}\left(n Z+\sum_{i=1}^{l} Z_{B_{i}}+Z_{E}+Z_{k-1}\right)+1=-A_{1} \cdot Z_{E}-2+1>2 .
\end{aligned}
$$


Under these conditions

$$
\begin{aligned}
\tau_{k}: S \otimes_{\mathrm{C}} H^{0} & \left(M, \frac{\vartheta\left(-n Z-\sum_{i=1}^{l} Z_{B_{i}}-Z_{E}-Z_{k-1}\right)}{\vartheta\left(-n Z-\sum_{i=1}^{l} Z_{B_{i}}-Z_{E}-Z_{k}\right)}\right] \\
& \rightarrow H^{0}\left(M, \frac{\vartheta\left(-(n+1) Z-2 \Sigma_{i=1}^{l} Z_{B_{i}}-2 Z_{E}-Z_{k-1}\right)}{\vartheta\left(-(n+1) Z-2 \sum_{i=1}^{l} Z_{B_{i}}-2 Z_{E}-Z_{k}\right)}\right)
\end{aligned}
$$

is still surjective. Namely, consider the subspace $T$ of $S$ of sections which vanish at some given point, say $a \in A_{1} . T$ has codimension 1 in $S$. If all the elements of $T$ have a common zero at some point $b \neq a \in A_{1}$ or if all have a second order zero at $a$, then $T$, having codimension 2 in

$$
H^{0}\left[M, \vartheta\left(-\sum_{i=0}^{l} Z_{B_{i}}-Z_{E}\right) / \mathcal{O}\left(-\sum_{i=0}^{l} Z_{B_{i}}-Z_{E}-A_{i_{k}}\right)\right] \text {, }
$$

represents all sections of a suitable line bundle over $A_{1}$. Then $\tau_{k}$ is readily seen to be surjective, as in the proof of [19, Lemma 7.9, pp. 144-146], but more easily. If the elements of $T$ have no common zeros, then think of $T$ as codimension 1 subspace of the sections of a line bundle and replace $S$ by $T$ in the previous case. Eventually we see that $\tau_{k}$ is surjective when $\operatorname{dim} T=1$.

Also in (3.8),

$$
H^{0}\left[M, \vartheta\left(-\sum_{i=0}^{l} Z_{B_{i}}-Z_{E}\right) / \mathcal{O}\left(-\sum_{i=0}^{l} Z_{B_{i}}-Z_{E}-A_{i_{t+k}}\right)\right]
$$

must be replaced by subspace $R_{t+k}$ which is the image of

$$
\begin{aligned}
\varphi_{t+k}: H^{0} & {\left[M, \vartheta\left(-\sum_{i=0}^{l} Z_{B_{i}}-Z_{E}\right)\right] } \\
& \rightarrow H^{0}\left(M, \vartheta\left(-\sum_{i=0}^{l} Z_{B_{i}}-Z_{E}\right) / \theta\left(-\sum_{i=0}^{l} Z_{B_{i}}-Z_{E}-A_{i_{t+k}}\right)\right)
\end{aligned}
$$

if $\varphi_{t+k}$ is not surjective. By the proof of Theorem 3.12, case (ii), we know that $R_{t+k}$ has at most codimension 1 in

$$
H^{0}\left[M, \vartheta\left(-\sum_{i=0}^{l} Z_{B_{i}}-Z_{E}\right) / \vartheta\left(-\sum_{i=0}^{l} Z_{B_{i}}-Z_{E}-A_{i_{i+k}}\right)\right] .
$$

Moreover, the elements of $R_{t+k}$ have no common zeros as sections of the line bundle on $A_{i_{t+k}}$ associated to

$$
\vartheta\left(-\sum_{i=0}^{l} Z_{B_{i}}-Z_{E}\right) / \theta\left(-\sum_{i=0}^{l} Z_{B_{i}}-Z_{E}-A_{i_{t+k}}\right) \text {. }
$$


We claim that if $\varphi_{t+k}$ is not surjective, then

$$
\operatorname{dim} H^{0}\left(M, \theta\left(-n Z-\sum_{i=1}^{l} Z_{B_{t}}-Z_{E}-Z_{t+k-1}\right) / \theta\left(-n Z-\sum_{i=1}^{l} Z_{B_{t}}-Z_{E}-Z_{t+k}\right)\right)>2 .
$$

Since $\varphi_{t+k}$ is not surjective, it follows from the proof of case (ii) of Theorem 3.12 that

$$
-A_{i_{k+1}} \cdot\left(\sum_{i=0}^{l} Z_{B_{i}}+Z_{E}\right)>1
$$

We will prove that actually

$$
-A_{i_{k+1}}\left(\sum_{i=0}^{l} Z_{B_{i}}+Z_{E}\right) \neq 1
$$

For if $-A_{i_{k+1}} \cdot\left(\sum_{i=0}^{l} Z_{B_{i}}+Z_{E}\right)=1$, then

$$
H^{0}\left[M, \vartheta\left(-\sum_{i=0}^{l} Z_{B_{i}}-Z_{E}\right) / \mathcal{O}\left(-\sum_{i=0}^{l} Z_{B_{i}}-Z_{E}-A_{i_{k+1}}\right)\right]=\mathbf{C}^{2} .
$$

An inductive argument, as in the proof of case (ii) of Theorem 3.12, will show that there exists $f \in H^{0}\left(M, \theta\left(-\sum_{i=0}^{l} Z_{B_{i}}-Z_{E}\right)\right)$ such that the image of $f$ in

$$
H^{0}\left[M, \vartheta\left(-\sum_{i=0}^{l} Z_{B_{i}}-Z_{E}\right) / \mathcal{O}\left(-\sum_{i=0}^{l} Z_{B_{i}}-Z_{E}-A_{i_{k+i}}\right)\right]
$$

as section of the line bundle associated to

$$
\mathcal{O}\left(-\sum_{i=0}^{l} Z_{B_{i}}-Z_{E}\right) / \mathcal{O}\left(-\sum_{i=0}^{l} Z_{B_{i}}-Z_{E}-A_{i_{k+l}}\right)
$$

has no zero on $A_{i_{k+1}} \cap A_{i_{k+1-1}}$. Hence, the image of $f$ cannot be in the image of $H^{0}\left[M, \vartheta\left(-\sum_{i=0}^{l} Z_{B_{i}}-Z_{E}-Z_{k+t-1}\right) / \theta\left(-\sum_{i=0}^{l} Z_{B_{i}}-Z_{E}-Z_{k+t}\right)\right]=\mathbf{C}$

which is injected into

$$
H^{0}\left[M, \vartheta\left(-\sum_{i=0}^{l} Z_{B_{i}}-Z_{E}\right) / \theta\left(-\sum_{i=0}^{l} Z_{B_{i}}-Z_{E}-A_{i_{k+l}}\right)\right]
$$

via the natural map and which is contained in $R_{t+k}$. Hence $\varphi_{t+k}$ is surjective. This contradicts our assumption. We conclude that

$$
-A_{i_{k+1}}\left(\sum_{i=0}^{l} Z_{B_{i}}+Z_{E}\right)>1
$$

and, hence,

$$
\operatorname{dim} H^{0}\left(M, \theta\left(-n Z-\sum_{i=1}^{l} Z_{B_{i}}-Z_{E}-Z_{t+k-1}\right) / O\left(-n Z-\sum_{i=1}^{l} Z_{B_{i}}-Z_{E}-Z_{t+k}\right)\right)>2
$$


Now repeating the argument above, we get

$$
\begin{aligned}
\tau_{t+k}: R_{t+k} \otimes_{\mathrm{C}} & H^{0}\left[M, \frac{\vartheta\left(-n Z-\sum_{i=1}^{l} Z_{B_{i}}-Z_{E}-Z_{t+k-1}\right)}{\vartheta\left(-n Z-\sum_{i=1}^{l} Z_{B_{i}}-Z_{E}-Z_{t+k}\right)}\right] \\
& \rightarrow H^{0}\left(M, \frac{\vartheta\left(-(n+1) Z-2 \Sigma_{i=1}^{l} Z_{B_{i}}-2 Z_{E}-Z_{t+k-1}\right)}{\vartheta\left(-(n+1) Z-2 \sum_{i=1}^{l} Z_{B_{i}}-2 Z_{E}-Z_{t+k}\right)}\right)
\end{aligned}
$$

is surjective.

In case $E=A_{1}=Z_{E}$ is an elliptic curve, we know that

$$
\begin{array}{r}
H^{0}\left(M, \frac{\theta\left(-\Sigma_{i=0}^{l} Z_{B_{i}}-Z_{E}\right)}{\theta\left(-\Sigma_{i=0}^{l} Z_{B_{i}}-Z_{E}-A_{1}\right)}\right) \otimes H^{0}\left(M, \frac{\theta\left(-n Z-\Sigma_{i=1}^{l} Z_{B_{i}}-Z_{E}\right)}{\theta\left(-n Z-\Sigma_{i=1}^{l} Z_{B_{i}}-Z_{E}-Z_{1}\right)}\right) \\
\rightarrow H^{0}\left(M, \frac{\theta\left(-(n+1) Z-2 \Sigma_{i=1}^{l} Z_{B_{i}}-2 Z_{E}\right)}{\theta\left(-(n+1) Z-2 \Sigma_{i=1}^{l} Z_{B_{i}}-2 Z_{E}-Z_{1}\right)}\right)
\end{array}
$$

is surjective. This is shown in [30]. The result follows from the proof above and the proof of case (i).

in case (iii), the proof of case (i) fails only because

$$
H^{0}\left(M, \theta\left(-n Z-\sum_{i=1}^{l} Z_{B_{i}}-Z_{E}-Z_{k-1}\right) / \mathcal{O}\left(-n Z-\sum_{i=1}^{l} Z_{B_{i}}-Z_{E}-Z_{k}\right)\right)=0 .
$$

We can still get

$$
H^{0}\left(M, \frac{\vartheta\left(-(n+1) Z-2 \sum_{i=1}^{l} Z_{B_{i}}-2 Z_{E}-Z_{k-1}\right)}{\vartheta\left(-(n+1) Z-2 \sum_{i=1}^{l} Z_{B_{i}}-2 Z_{E}-Z_{k}\right)}\right)
$$

as an image as follows: There are two subcases. First, suppose that $A_{1}$ can be chosen so that $A_{1} \cdot Z_{E}<0$ and $e_{1}>1$ in $E=\sum e_{i} A_{i}$. In this subcase $Z_{E}=E$. Then choose a computation sequence for $Z$ with $A_{i_{1}} \cdot Z_{E}<0, E=Z_{k}$, $A_{1}=A_{i_{k}}$ and with $Z_{q}, q<k$, such that $A_{i_{q}}=A_{1}, A_{1} \ell \operatorname{supp}\left(E-A_{1}-Z_{q}\right)$ and $A_{i} \cdot Z_{q-1} \leqslant 0, i \neq 1, A_{i} \subseteq \operatorname{supp} E$. Such a computation sequence can be formed by letting $A_{i_{j}}=A_{1}$ only when $A_{i_{j}} \subseteq|E|$ cannot be chosen otherwise. Then also $0, Z_{q}-Z_{q-1}, Z_{q+1}-Z_{q-1}, \ldots, Z_{k}-Z_{q-1}$ is part of a computation sequence for $Z_{E}=Z_{k}$, which, by Corollary 2.3, can be continued to terminate at $A_{i_{1}}$. Recall that $A_{i_{1}} \cdot Z_{E}<0$ by construction. Hence

$$
H^{1}\left[M, \vartheta\left(-n Z-\sum_{i=1}^{l} Z_{B_{i}}-Z_{E}-\left(Z_{k}-Z_{q-1}\right)\right)\right]=0
$$


and also

$$
H^{1}\left[M, \vartheta\left(-\sum_{i=0}^{l} Z_{B_{i}}-Z_{E}-Z_{q}\right)\right]=0
$$

In place of (3.8), we use

$$
\begin{aligned}
H^{0}\left(M, \frac{\theta\left(-\Sigma_{i=0}^{l} Z_{B_{i}}-Z_{E}-Z_{q-1}\right)}{\theta\left(-\Sigma_{i=0}^{l} Z_{B_{i}}-Z_{E}-Z_{q}\right)}\right) \\
\\
\otimes H^{0}\left(M, \frac{\theta\left(-n Z-\Sigma_{i=1}^{l} Z_{B_{i}}-Z_{E}-\left(Z_{k-1}-Z_{q-1}\right)\right)}{\theta\left(-n Z-\Sigma_{i=1}^{l} Z_{B_{i}}-Z_{E}-\left(Z_{k}-Z_{q-1}\right)\right)}\right) \\
\rightarrow H^{0}\left(M, \frac{\theta\left(-(n+1) Z-2 \Sigma_{i=1}^{l} Z_{B_{i}}-2 Z_{E}-Z_{k-1}\right)}{\theta\left(-(n+1) Z-2 \Sigma_{i=1}^{l} Z_{B_{i}}-2 Z_{E}-Z_{k}\right)}\right) .
\end{aligned}
$$

Look at the following commutative diagram:

\section{Diagram H (page 318)}

with the vertical column on the right exact. Our result follows easily.

In the other subcase, there must be $A_{1}, A_{2}$ and $A_{3}$ all distinct, such that $A_{i} \cdot Z_{E}<0, \quad 1 \leqslant i<3$, and $e_{i}=1, \quad 1<i<3$. Choose a computation sequence for $Z_{E}$ with $E=Z_{k}$ such that $A_{i_{1}} \cdot A_{1}>0, A_{i_{k}}=A_{1}$, and such that when $Z_{q}$ with $q<k, A_{i q}=A_{2}$ is reached, $A_{i} \cdot Z_{q-1}<0$ for $i \neq 1,2$. We may suppose $A_{3} \subset$ supp $Z_{q-1}$, for otherwise we reverse the roles of $A_{2}$ and $A_{3}$. Since $A_{i_{1}} \cdot A_{1}>0$ and $e_{1}=1, Z_{q-1}+A_{1}$ is part of a computation sequence for $Z_{E} \cdot 0, Z_{q}-Z_{q-1}, \ldots, Z_{k}-Z_{q-1}$ is also part of a computation sequence for $Z_{E}$. Therefore

$$
H^{1}\left[M, \theta\left(-\sum_{i=0}^{l} Z_{B_{i}}-Z_{E}-Z_{q-1}-A_{1}\right)\right]=0
$$

and

$$
H^{1}\left[M, \vartheta\left(-n Z-\sum_{i=1}^{l} Z_{B_{i}}-Z_{E}-\left(Z_{k}-Z_{q-1}\right)\right)\right]=0
$$

by Proposition 2.7. In place of (3.8), we use

$$
\begin{aligned}
H^{0}\left(M, \frac{\theta\left(-G-Z_{E}-Z_{g-1}\right)}{\theta\left(-G-Z_{E}-Z_{q-1}-A_{1}\right)}\right) & \otimes H^{0}\left(M, \frac{\theta\left(-n Z-B-Z_{E}-\left(Z_{k-1}-Z_{q-1}\right)\right)}{\theta\left(-n Z-B-Z_{E}-\left(Z_{k}-Z_{q-1}\right)\right)}\right) \\
& \rightarrow H^{0}\left(M, \frac{\theta\left(-(n+1) Z-2 B-2 Z_{E}-Z_{k-1}\right)}{\theta\left(-(n+1) Z-2 B-2 Z_{E}-Z_{k}\right)}\right)
\end{aligned}
$$

where $G=\sum_{i=0}^{l} Z_{B_{i}}$ and $B=\sum_{i=1}^{l} Z_{B_{i}}$.

Look at the following commutative diagram: 


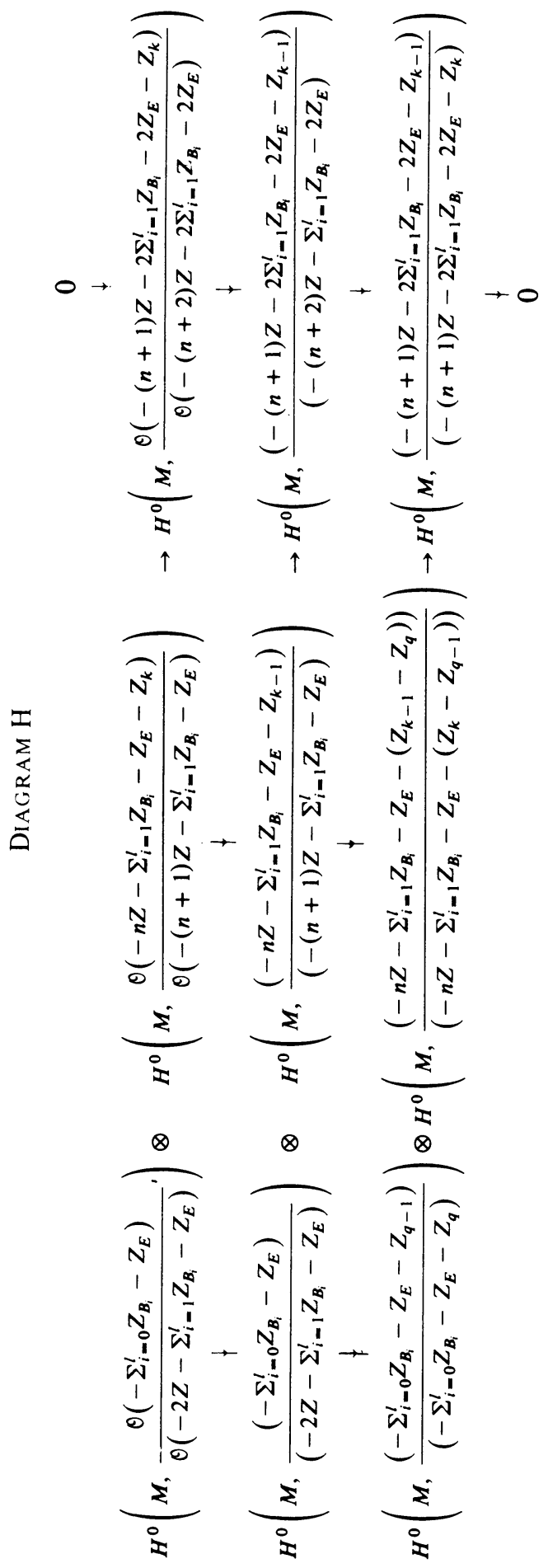




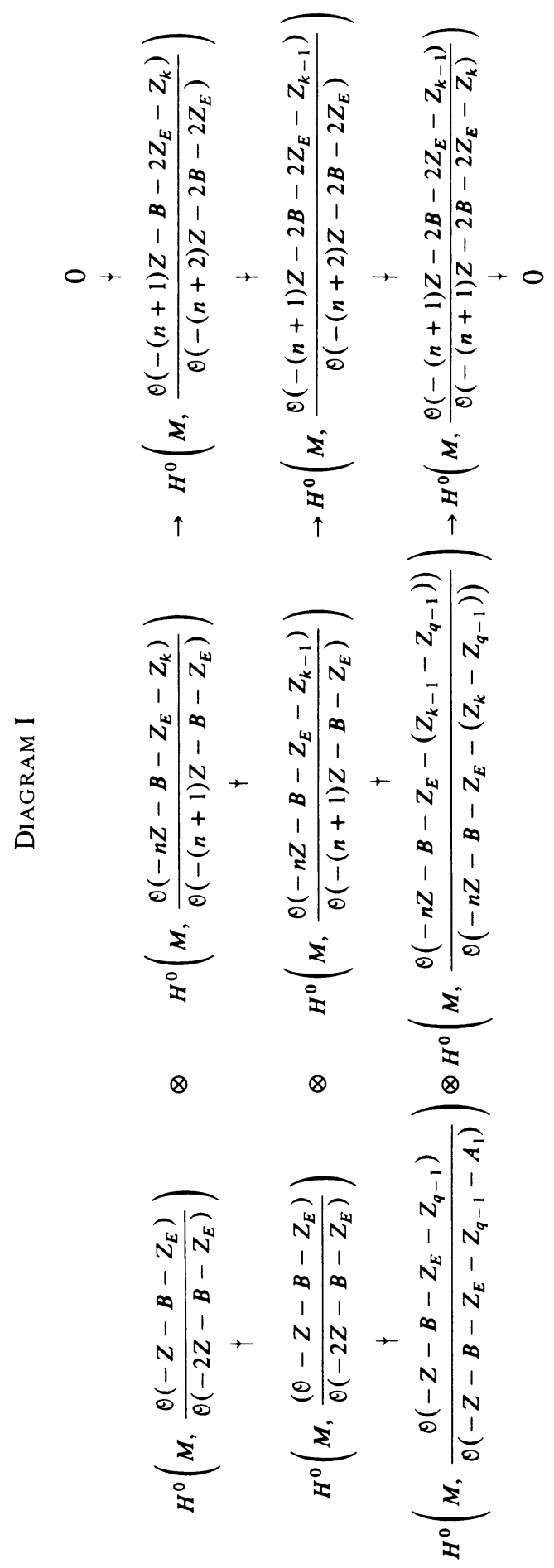


with the vertical column on the right exact. The results follow readily. This completes the proof of Step 1.

Step 2. We are going to show that

(3.9) We can get

$$
H^{0}\left[M, \mathcal{O}(-(n+1) Z) / \mathcal{O}\left(-(n+1) Z-2 \sum_{i=1}^{l} Z_{B_{i}}-2 Z_{E}\right)\right]
$$

as an image for all $n>1$.

The proof of (3.9) breaks up into two subcases.

(i) supp $E$ has more than one irreducible component.

(ii) supp $E$ is a nonsingular elliptic curve.

In case (i), choose a computation sequence for $Z$ of the following form.

$$
\begin{aligned}
Z_{0}=0, Z_{1}= & A_{i_{1}} \cdots Z_{k}=Z_{k-1}+A_{i_{k}}=E, \ldots, \\
& Z_{r_{0}}=Z_{E}, \ldots, Z_{r_{1}}=Z_{B_{l}}, \ldots, Z_{r_{l}}=Z_{B_{1}}, \ldots, Z_{r_{I+1}}=Z_{B_{0}}=Z,
\end{aligned}
$$

where $A_{i_{k}}=A_{1}$ and $A_{1} \cdot Z_{E}<0$. Consider

$$
\begin{gathered}
\gamma_{B_{0} \ldots, B_{h} ;}: H^{0}\left(M, \frac{\theta(-Z)}{\theta\left(-Z-A_{i j}\right)}\right) \otimes_{C} H^{0}\left(M, \frac{\theta\left(-n Z-\Sigma_{i=1}^{h} Z_{B_{i}}-Z_{j-1}\right)}{\theta\left(-n Z-\Sigma_{i=1}^{h} Z_{B_{i}}-Z_{j}\right)}\right) \\
\rightarrow H^{0}\left(M, \frac{\left(-(n+1) Z-\Sigma_{i-1}^{h} Z_{B_{i}}-Z_{j-1}\right)}{\left(-(n+1) Z-\Sigma_{i=1}^{h} Z_{B_{i}}-Z_{j}\right)}\right), \\
0<j<r_{h+1},-1<h<l-1,
\end{gathered}
$$

$$
\begin{aligned}
& \gamma_{B_{0} \ldots, B_{h j}:}^{\prime} H^{0}\left(M, \frac{\theta(-Z)}{\theta\left(-Z-A_{j}\right)}\right) \\
& \otimes_{C} H^{0}\left(M, \frac{\theta\left(-n Z-\Sigma_{i-1}^{l} Z_{B_{i}}-Z_{E}-\Sigma_{i-1}^{h} Z_{B_{i}}-Z_{j-1}\right)}{\theta\left(-n Z-\Sigma_{i-1}^{l} Z_{B_{i}}-Z_{E}-\Sigma_{i=1}^{h} Z_{B_{i}}-Z_{j-1}\right)}\right) \\
& \rightarrow H^{0}\left(M, \frac{\theta\left(-(n+1) Z-\Sigma_{i-1}^{l} Z_{B_{i}}-Z_{E}-\Sigma_{i=1}^{h} Z_{B_{i}}-Z_{j-1}\right)}{\theta\left(-(n+1) Z-\Sigma_{i=1}^{l} Z_{B_{i}}-Z_{E}-\Sigma_{i-1}^{h} Z_{B_{i}}-Z_{j}\right)}\right), \\
& 0<j<r_{h+1},-1<h<l-1 .
\end{aligned}
$$

To show that (3.9) is true, it will suffice to show that $\gamma_{B_{0}, \ldots, B_{h} ;}, \gamma_{B_{0}, \ldots, B_{h} ; j}^{\prime}$ are surjective for all $0<j<\gamma_{h+1},-1<h<l-1$. Consider the following sheaf exact sequences:

$$
\begin{aligned}
0 \rightarrow & \frac{\theta\left(-n Z-G_{h}-Z_{j}\right)}{\theta\left(-n Z-2 G_{l}-2 Z_{E}\right)} \rightarrow \frac{\theta\left(-n Z-G_{h}-Z_{j-1}\right)}{\theta\left(-n Z-2 G_{l}-2 Z_{E}\right)} \rightarrow \frac{\theta\left(-n Z-G_{h}-Z_{j-1}\right)}{\theta\left(-n Z-G_{h}-Z_{j}\right)} \rightarrow 0, \\
0 & \rightarrow \frac{\theta\left(-n Z-G_{l}-Z_{E}-G_{h}-Z_{j}\right)}{\theta\left(-n Z-2 G_{l}-2 Z_{E}\right)} \rightarrow \frac{\theta\left(-n Z-G_{l}-Z_{E}-G_{h}-Z_{j-1}\right)}{\theta\left(-n Z-2 G_{l}-2 Z_{E}\right)} \\
& \rightarrow \frac{\theta\left(-n Z-G_{l}-Z_{E}-G_{h}-Z_{j-1}\right)}{\theta\left(-n Z-G_{l}-Z_{E}-G_{h}-Z_{j}\right)} \rightarrow 0, \quad-1<h<l-1,1<j<r_{l-h},
\end{aligned}
$$


where we denote $G_{-1}=0, Z_{0}=0, G_{h}=\sum_{i=1}^{h} Z_{B_{i}}$. We claim that

$$
\begin{aligned}
H^{0}[ & \left.M, \vartheta\left(-n Z-\sum_{i=1}^{h} Z_{B_{i}}-Z_{j-1}\right) / \theta\left(-n Z-2 \sum_{i=1}^{l} Z_{B_{i}}-2 Z_{E}\right)\right] \\
& \rightarrow H^{0}\left\{M, \vartheta\left(-n Z-\sum_{i=1}^{h} Z_{B_{i}}-Z_{j-1}\right) / \theta\left(-n Z-\sum_{i=1}^{h} Z_{B_{i}}-Z_{j}\right)\right]
\end{aligned}
$$

are surjective for all $-1<h<l-1$ and $0<j<r_{h+1}$. The Chern class of line bundle associated to

$$
\vartheta\left(-n Z-\sum_{i=1}^{h} Z_{B_{i}}-Z_{j-1}\right) / \theta\left(-n Z-\sum_{i=1}^{h} Z_{B_{i}}-Z_{j}\right)
$$

is

$$
-A_{i j}\left(n Z+\sum_{i=1}^{h} Z_{B_{i}}+Z_{j-1}\right)=-A_{i j} \cdot Z_{j-1}
$$

which is less than zero for $j>1$ and 0 for $j=1$. Therefore for $j>1$, the claim is trivially true because

$$
H^{0}\left[M, \theta\left(-n Z-\sum_{i=1}^{h} Z_{B_{i}}-Z_{j-1}\right) / \theta\left(-n Z-\sum_{i=1}^{h} Z_{B_{i}}-Z_{j}\right)\right]=0
$$

For $j=0$,

$$
H^{0}\left[M, \theta\left(-n Z-\sum_{i=1}^{h} Z_{B_{i}}\right) / \theta\left(-n Z-\sum_{i=1}^{h} Z_{B_{i}}-Z_{1}\right)\right]=\mathbf{C} .
$$

By Proposition 3.13, we know that there exists

$$
f \in H^{0}\left[M, \theta\left(-n Z-\sum_{i=1}^{h} Z_{B_{i}}\right)\right]
$$

such that the image of $f$ in

$$
H^{0}\left[M, \theta\left(-n Z-\sum_{i=1}^{h} Z_{B_{i}}\right) / \theta\left(-n Z-\sum_{i=1}^{h} Z_{B_{i}}-Z_{1}\right)\right]
$$

is nonzero. It follows that

$$
H^{0}\left(M, \frac{\theta\left(-n Z-\Sigma_{i=0}^{h} Z_{B_{1}}\right)}{\theta\left(-n Z-2 \Sigma_{i=1}^{l} Z_{B_{l}}-2 Z_{E}\right)}\right) \rightarrow H^{0}\left(M, \frac{\theta\left(-n Z-\Sigma_{i-1}^{h} Z_{B_{i}}\right)}{\theta\left(-n Z-\Sigma_{i=1}^{h} Z_{B_{i}}-Z_{1}\right)}\right)
$$

is surjective. We next prove that

$$
H^{1}\left[M, \frac{\Theta\left(-n Z-\Sigma_{i=1}^{l} Z_{B_{i}}-Z_{E}-\sum_{i=1}^{h} Z_{B_{i}}-Z_{j-1}\right)}{\Theta\left(-n Z-\Sigma_{i=1}^{l} Z_{B_{i}}-Z_{E}-\sum_{i=1}^{h} Z_{B_{i}}-Z_{j}\right)}\right]=0
$$

for $-1<h<l-1$ and $0<j<r_{h+1}$. 


$$
\theta\left(-n Z-\sum_{i=1}^{l} Z_{B_{i}}-Z_{E}-\sum_{i=1}^{h} Z_{B_{i}}-Z_{j-1}\right) / \theta\left(-n Z-\sum_{i=1}^{l} Z_{B_{i}}-Z_{E}-\sum_{i=1}^{h} Z_{B_{i}}-Z_{j}\right)
$$

is the sheaf of germs of sections of a line bundle over $A_{i}$ of Chern class $-A_{i j} \cdot\left(n Z+\sum_{i=1}^{l} Z_{B_{i}}+Z_{E}+\sum_{i=1}^{h} Z_{B_{i}}+Z_{j-1}\right)$. Recall that by construction $A_{i_{k}} \cdot Z_{E}<-1$. Therefore,

$$
-A_{i} \cdot\left(n Z+\sum_{i=1}^{l} Z_{B_{i}}+\sum_{i=1}^{h} Z_{B_{i}}+Z_{j-1}\right)>-1 .
$$

By the Riemann-Roch Theorem, we have

$$
H^{1}\left[M, \frac{\Theta\left(-n Z-\sum_{i=1}^{l} Z_{B_{i}}-Z_{E}-\sum_{i=1}^{h} Z_{B_{i}}-Z_{j-1}\right)}{\Theta\left(-n Z-\sum_{i=1}^{l} Z_{B_{i}}-Z_{E}-\sum_{i=1}^{h} Z_{B_{i}}-Z_{j}\right)}\right]=0 .
$$

Now the usual long cohomology exact sequence argument will show that

$$
\begin{aligned}
H^{0}\left[M, \frac{\theta\left(-n Z-\sum_{i=1}^{l} Z_{B_{i}}-Z_{E}-\sum_{i=1}^{h} Z_{B_{i}}-Z_{j-1}\right)}{\theta\left(-n Z-2 \Sigma_{i=1}^{l} Z_{B_{i}}-2 Z_{E}\right)}\right] \\
\quad \rightarrow H^{0}\left[M, \frac{\theta\left(-n Z-\Sigma_{i=1}^{l} Z_{B_{i}}-Z_{E}-\sum_{i=1}^{h} Z_{B_{i}}-Z_{k-1}\right)}{\theta\left(-n Z-\sum_{i=1}^{l} Z_{B_{i}}-Z_{E}-\sum_{i=1}^{h} Z_{B_{i}}-Z_{k}\right)}\right]
\end{aligned}
$$

is surjective for all $-1<h<l-1$ and $0<j<r_{h+1}$. So far we have proved

$$
H^{0}\left[M, \theta(-n Z) / \mathcal{O}\left(-n Z-2 \sum_{i=1}^{l} Z_{B_{i}}-2 Z_{E}\right)\right]
$$

can be written via successive quotients.

$$
\begin{aligned}
& 0 \rightarrow H^{0}\left(M, \frac{\theta\left(-n Z-G_{h}-Z_{j}\right)}{\theta\left(-n Z-2 G_{l}-2 Z_{E}\right)}\right) \rightarrow H^{0}\left(M, \frac{\theta\left(-n Z-G_{h}-Z_{j-1}\right)}{\theta\left(-n Z-2 G_{l}-2 Z_{E}\right)}\right) \\
& \rightarrow H^{0}\left(M, \frac{\theta\left(-n Z-G_{h}-Z_{j-1}\right)}{\theta\left(-n Z-G_{h}-Z_{j}\right)}\right) \rightarrow 0, \\
& 0 \rightarrow H^{0}\left(\frac{\theta\left(-n Z-G_{l}-Z_{E}-G_{h}-Z_{j}\right)}{\theta\left(-n Z-2 G_{l}-2 Z_{E}\right)}\right) \rightarrow H^{0}\left(M, \frac{\theta\left(-n Z-G_{l}-Z_{E}-G_{h}-Z_{j-1}\right)}{\theta\left(-n Z-2 G_{l}-2 Z_{E}\right)}\right) \\
& \rightarrow H^{0}\left(M, \frac{\theta\left(-n Z-G_{l}-Z_{E}-G_{h}-Z_{j-1}\right)}{\theta\left(-n Z-G_{l}-Z_{E}-G_{h}-Z_{j}\right)}\right) \rightarrow 0,
\end{aligned}
$$

$-1<h<l-1,1<j<r_{l-h}$, where we denote $G_{-1}=0, Z_{0}=0, G_{h}=$ $\sum_{i=1}^{h} Z_{B_{i}}$.

By the proof of Theorem 3.12, we know that

$$
H^{0}(M, \theta(-Z)) \rightarrow H^{0}\left(M, \theta(-Z) / \theta\left(-Z-A_{i j}\right)\right)
$$


is surjective for all $A_{i} \subseteq B_{1}$. Since $n>2$,

$$
\begin{aligned}
H^{1}\left(M, O\left(-n Z-\sum_{i=1}^{h} Z_{B_{i}}-Z_{j}\right)\right) & =0 \\
& =H^{1}\left(M, O\left(-n Z-\sum_{i=1}^{l} Z_{B_{i}}-Z_{E}-\sum_{i=1}^{h} Z_{B_{i}}-Z_{j}\right)\right)
\end{aligned}
$$

by Proposition 2.8. Now look at the following commutative diagrams:

Diagram J (page 324),

$-1<h<l-1,1<j<r_{l-h}$, where we denote $G_{h}=\sum_{i=1}^{h} Z_{B_{i}}, G_{-1}=0$, $Z_{0}=0$. Thus if $\gamma_{B_{0}} \ldots, B_{h} ; j$ and $\gamma_{B_{0}}^{\prime} \ldots, B_{h} ; j$ are surjective $\forall-1<h<l-1$, $0<j<r_{h+1}$, then (3.9) is true.

By the Riemann-Roch Theorem, the target space of $\gamma_{B_{0}} \ldots, B_{h} j$ is nonzero only if $j=1$. In that case $-A_{i_{1}} \cdot Z=0$ and $-A_{i_{1}} \cdot\left(n Z+\sum_{i=1}^{h} Z_{B_{l}}\right)=0$. Hence $\gamma_{B_{0}} \ldots, B_{h, j}$ is surjective for all $h$ and $j$. Suppose that the target space of $\gamma_{B_{0}}^{\prime} \ldots, B_{h, j}$ is nonzero, i.e.,

$$
-A_{i j} \cdot\left((n+1) Z+\sum_{i=1}^{l} Z_{B_{i}}+Z_{E}+\sum_{i=1}^{h} Z_{B_{i}}+Z_{j-1}\right)>0
$$

We need

$$
-A_{i j} \cdot Z>0 \text { and }-A_{i j} \cdot\left(n Z+\sum_{i=1}^{l} Z_{B_{i}}+Z_{E}+\sum_{i=1}^{h} Z_{B_{i}}+Z_{j-1}\right)>0 \text {. }
$$

But this is obvious because $A_{i,} \cdot Z=0$ for $A_{i,} \subseteq B_{1}$.

In case (ii), $E=Z_{E}=A_{1}$ is a nonsingular elliptic curve. We first show that

$$
\begin{gathered}
H^{0}\left(M, \frac{\theta(-Z)}{\theta\left(-Z-A_{1}\right)}\right) \otimes H^{0}\left[M, \frac{\theta\left(-n Z-\sum_{i=1}^{h} Z_{B_{i}}\right)}{\theta\left(-n Z-\sum_{i=1}^{h} Z_{B_{i}}-Z_{1}\right)}\right] \\
\rightarrow H^{0}\left[M, \frac{\theta\left(-(n+1) Z-\sum_{i=1}^{h} Z_{B_{i}}\right)}{\theta\left(-(n+1) Z-\Sigma_{i=1}^{h} Z_{B_{j}}-Z_{1}\right)}\right]
\end{gathered}
$$

is surjective. The Chern classes of the line bundles associated to

$$
\Theta(-Z) / O\left(-Z-A_{1}\right), \quad \theta\left(-n Z-\sum_{i=0}^{h} Z_{B_{i}}\right) / \theta\left(-n Z-\sum_{i=1}^{h} Z_{B_{i}}-Z_{1}\right)
$$

and

$$
\mathcal{O}\left(-(1+n) Z-\sum_{i=1}^{h} Z_{B_{i}}\right) / \mathcal{O}\left(-(n+1) Z-\sum_{i=1}^{h} Z_{B_{i}}-Z_{1}\right)
$$




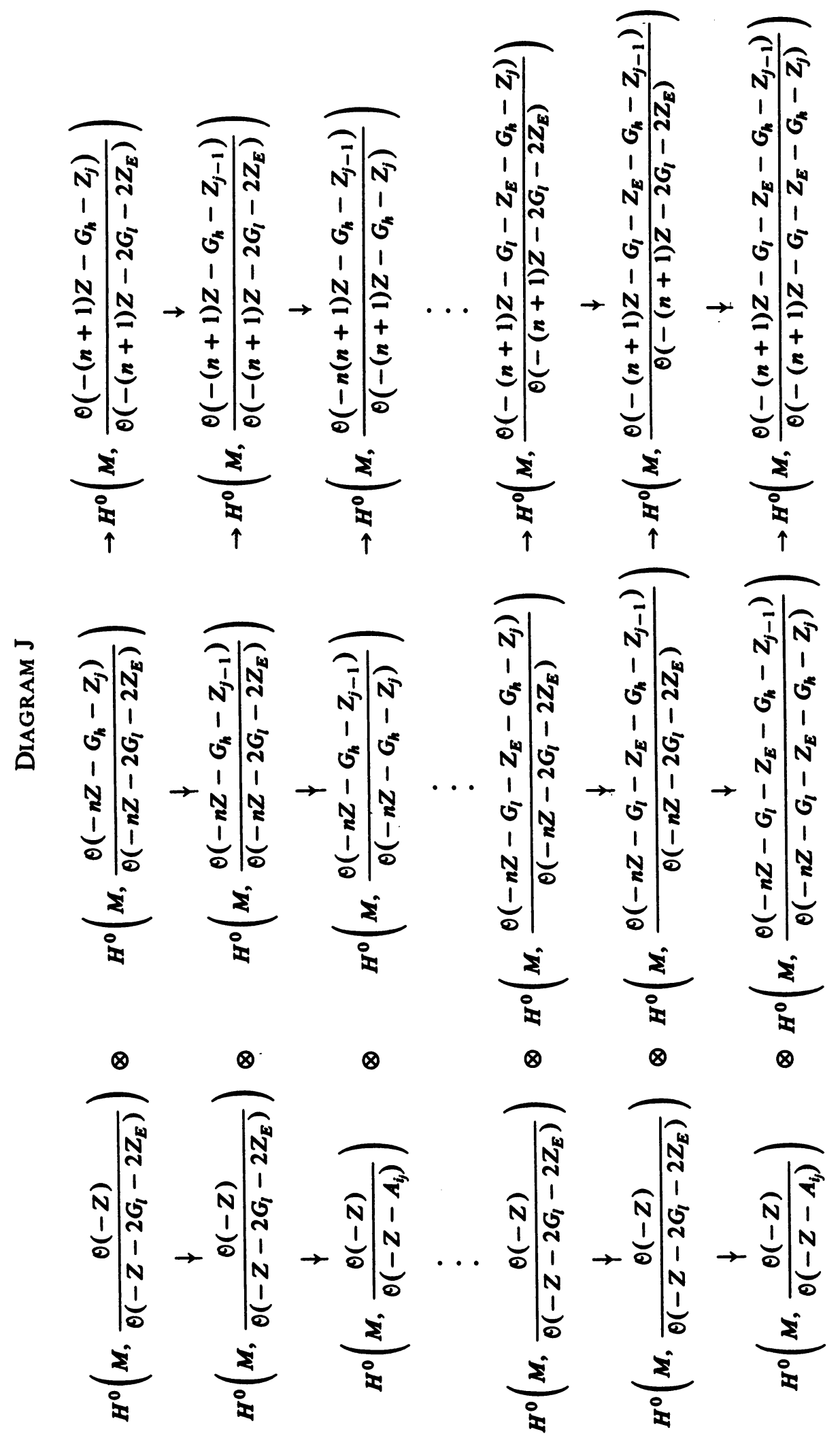


respectively, are equal to zero. By Proposition 3.13, there exist

$$
\begin{gathered}
f_{0} \in H^{0}(M, \theta(-Z)), \quad f_{n, h} \in H^{0}\left[M, \vartheta\left(-n Z-\sum_{i=0}^{h} Z_{B_{i}}\right)\right], \\
f_{n+1, h} \in H^{0}\left[M, \vartheta\left(-(n+1) Z-\sum_{i=1}^{h} Z_{B_{i}}\right)\right],
\end{gathered}
$$

the images of $f_{0}, f_{n, h}$ and $f_{n+1, h}$ in

$$
H^{0}\left(M, O(-Z) / O\left(-Z-A_{1}\right)\right), H^{0}\left(M, O\left(-n Z-\sum_{i=1}^{h} Z_{B_{1}}\right) / O\left(-n Z-\sum_{i=1}^{h} Z_{B_{1}}-Z_{1}\right)\right)
$$

and

$$
H^{0}\left[M, \vartheta\left(-(n+1) Z-\sum_{i=1}^{h} Z_{B_{i}}\right) / \mathcal{O}\left(-(n+1) Z-\sum_{i=1}^{h} Z_{B_{i}}-Z_{1}\right)\right] \text {, }
$$

respectively, are nonzero. It follows from the Riemann-Roch Theorem that

$$
\begin{gathered}
H^{0}\left(M, \theta(-Z) / \theta\left(-Z-A_{1}\right)\right) \simeq \mathrm{C} \\
H^{0}\left[M, \theta\left(-n Z-\sum_{i=1}^{h} Z_{B_{i}}\right) / \theta\left(-n Z-\sum_{i=1}^{h} Z_{B_{i}}-Z_{1}\right)\right] \simeq \mathrm{C}
\end{gathered}
$$

and

$$
H^{0}\left(M, O\left(-(n+1) Z-\sum_{i=1}^{h} Z_{B_{1}}\right) / \mathcal{O}\left(-(n+1) Z-\sum_{i=1}^{h} Z_{B_{i}}-Z_{1}\right)\right) \simeq \mathrm{C} .
$$

Hence the maps (3.11) are surjective. We next show that

$$
\begin{gathered}
H^{0}\left(M, \frac{\theta(-Z)}{\theta\left(-Z-A_{1}\right)}\right) \otimes H^{0}\left(M, \frac{\theta\left(-n Z-\Sigma_{i=1}^{l} Z_{B_{1}}-Z_{E}-\Sigma_{i=1}^{h} Z_{B_{i}}\right)}{\theta\left(-n Z-\Sigma_{i=1}^{l} Z_{B_{i}}-Z_{E}-\Sigma_{i=1}^{h} Z_{B_{i}}-Z_{1}\right)}\right) \\
\rightarrow H^{0}\left(M, \frac{\theta\left(-(n+1) Z-\Sigma_{i=1}^{l} Z_{B_{i}}-Z_{E}-\Sigma_{i=1}^{h} Z_{B_{i}}\right)}{\theta\left(-(n+1) Z-\Sigma_{i=1}^{l} Z_{B_{i}}-Z_{E}-\Sigma_{i=1}^{h} Z_{B_{i}}-Z_{1}\right)}\right)
\end{gathered}
$$

is surjective. The Chern class of the line bundle associated to

$$
\theta\left(-n Z-\sum_{i=1}^{l} Z_{B_{i}}-Z_{E}-\sum_{i=1}^{h} Z_{B_{1}}\right) / \theta\left(-n Z-\sum_{i=1}^{l} Z_{B_{i}}-Z_{E}-\sum_{i=1}^{h} Z_{B_{i}}-Z_{1}\right)
$$

is equal to

$$
-A_{1} \cdot\left(n Z+\sum_{i=1}^{l} Z_{B_{i}}+Z_{E}+\sum_{i=1}^{h} Z_{B_{i}}\right)=-A_{1} \cdot Z_{E}>3
$$




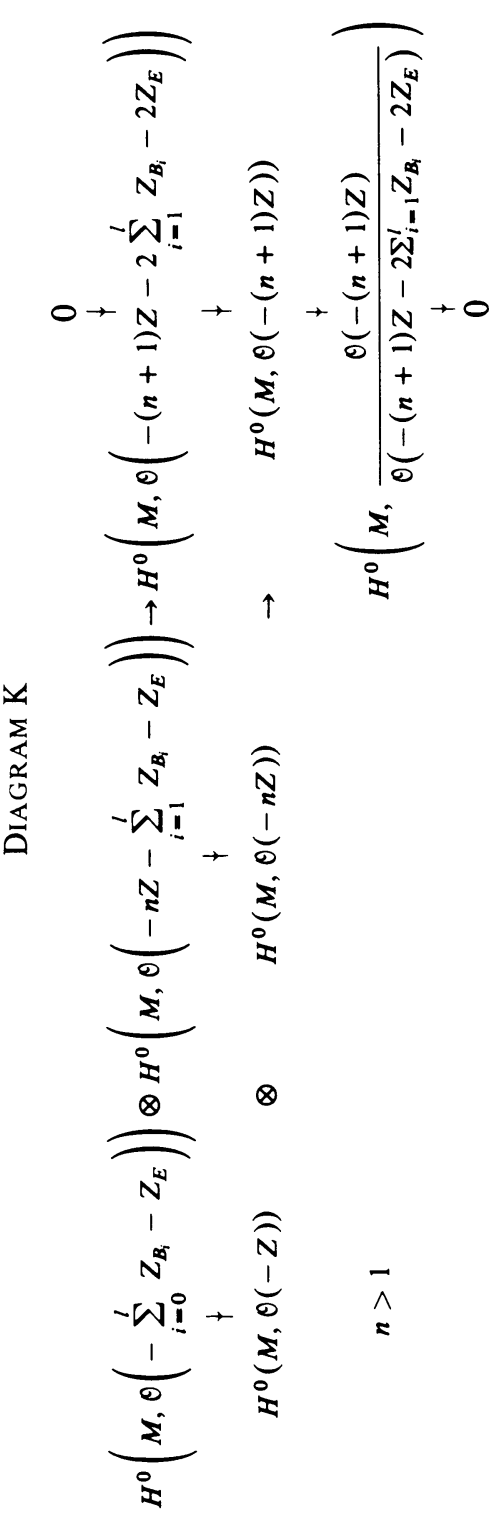


The Chern class of the line bundle associated to

$$
\theta\left(-(n+1) Z-\sum_{i=1}^{l} Z_{B_{i}}-Z_{E}-\sum_{i=1}^{h} Z_{B_{i}}\right) / \theta\left(-(n+1) Z-\sum_{i=1}^{h} Z_{B_{i}}-Z_{E}-\sum_{i=1}^{h} Z_{B_{i}}-Z_{1}\right)
$$

is equal to

$$
-A_{1}\left((n+1) Z+\sum_{i=1}^{l} Z_{B_{i}}+Z_{E}+\sum_{i=1}^{h} Z_{B_{i}}\right)=-A_{1} \cdot Z_{E}>3 .
$$

Therefore

$$
\begin{aligned}
\operatorname{dim} H^{0}\left[M, \frac{\theta\left(-n Z-\sum_{i=1}^{l} Z_{B_{i}}-Z_{E}-\sum_{i=1}^{h} Z_{B_{i}}\right)}{\Theta\left(-n Z-\sum_{i=1}^{l} Z_{B_{i}}-Z_{E}-\sum_{i=1}^{h} Z_{B_{i}}-Z_{1}\right)}\right] \\
=\operatorname{dim} H^{0}\left[M, \frac{\theta\left(-(n+1) Z-\sum_{i=1}^{l} Z_{B_{i}}-Z_{E}-\sum_{i=1}^{h} Z_{B_{i}}\right)}{\Theta\left(-(n+1) Z-\sum_{i=1}^{l} Z_{B_{i}}-Z_{E}-\sum_{i=1}^{h} Z_{B_{i}}-Z_{1}\right)}\right] .
\end{aligned}
$$

By what we have already proved, the line bundle associated to $\theta(-Z) / \theta\left(-Z-A_{1}\right)$ is a trivial line bundle. Hence the maps in (3.12) are surjective. The rest of the proof is the same as case (i).

Step 3. Consider the commutative diagram:

Diagram K (page 326)

with the column sequence on the right exact. The map of the first row is surjective by Step 1. It follows that the map of second row is also surjective.

Let us make the following observation. The only thing that we need $n>1$ in the proof of the map

$$
H^{0}(M, \theta(-Z)) \otimes H^{0}(M, \vartheta(-n Z)) \rightarrow H^{0}(M, \theta(-(n+1) Z))
$$

being surjective is to get vanishing first cohomology

$$
\begin{aligned}
H^{1}\left[M, \vartheta\left(-n Z-\sum_{i=1}^{l} Z_{B_{i}}-Z_{E}-\sum_{i=1}^{h} Z_{B_{i}}-Z_{j}\right)\right] \\
-1<h<l-1,0<j<r_{h+1} .
\end{aligned}
$$

If the length of the elliptic sequence is equal to two, this is automatically satisfied by Proposition 2.7. Q.E.D.

\section{REFERENCES}

1. S. Abhyanker, Resolution of singularities of embedded algebraic surfaces, Academic Press, New York, 1966.

2. M. Artin, On isolated rational singularities of surfaces, Amer. J. Math. 88 (1966), 129-136.

3. H. Bass, On the ubiquity of Gorenstein rings, Math. Z. 82 (1963), 8-28.

4. E. Brieskorn, Rationale Singularitäten komplexer Flächen, Invent. Math. 4 (1967/68), 336-358. 
5. Über die Auflösung gewisser Singularitäten von holomorphen Abbildungen, Math.

Ann. 166 (1966), 76-102.

6. P. DuVal, On isolated singularities of surfaces which do not affect the condition of adjunction, Proc. Cambridge Philos. Soc. 30 (1933/34), 453-491.

7. H. Grauert, Uber Modifikationen und exzeptionnelle analytische Mengen, Math. Ann. 146 (1962), 331-368.

8. H. Grauert and O. Riemenschneider, Verschwindungssätze für analytische Kohomologiegruppen auf komplexen Räumen, Springer, Berlin, 1970, pp. 97-109.

9. R. Gunning, Lectures on Riemann surfaces, Princeton Univ. Press, Princeton, N.J., 1966.

10. R. Gunning and H. Rossi, Analytic functions of several complex variables, Prentice-Hall, Englewood Cliffs, N.J., 1965.

11. H. Hironaka, Resolution of singularities of an algebraic variety over a field of characteristic zero. I, II, Ann. of Math. (2) 79 (1964), 108-326.

12. F. Hirzebruch, The Hilbert modular group, resolution of singularities at the cusps and related problems, Sém. N. Bourbaki (1970/71), Exp. 396.

13. __ Über vierdimensionale Riemannschen Flächen mehrdeutiger analytischer Funktionen von zwei komplexen Veränderlichen, Math. Ann. 126 (1953), 1-22.

14. F. Hirzebruch, W. Neumann and S. Koh, Differentiable manifolds and quadratic forms, Dekker, New York, 1971.

15. U. Karras, Dissertation, Bonn, 1973.

16. __ Eigenshaften der lokalen Ringe in zweidimensionaten Spetzen, Math. Ann. (to appear).

17. D. Kirby, The structure of an isolated multiple point of a surface. I, Proc. London Math. Soc. 6 (1956), 597-609.

18. _ The structure of an isolated multiple point of a surface. II, Proc. London Math. Soc. 7 (1957), 1-28.

19. H. Laufer, Normal two-dimensional singularities, Ann. of Math. Studies no. 71, Princeton Univ. Press, Princeton, N.J., 1971.

20. __ On rational singularities, Amer. J. Math. 94 (1972), 597-608.

21. Deformations of resolutions of two-dimensional singularities, Rice Univ. Studies 59 (1973), 53-96.

22. __ Taut two-dimensional singularities, Math. Ann. 205 (1973), 131-164.

23. __ On $\mu$ for surface singularities (to appear).

24. __ Minimally elliptic singularities, Amer. J. Math. (to appear).

25. J. Lipman, Rational singularities with applications to algebraic surfaces and unique factorization, Inst. Hautes Etudes Sci. Publ. Math. no. 36 (1969), 195-279.

26. D. Mumford, The topology of normal singularities of an algebraic surface and a criterion for simplicity, Inst. Hautes Etudes Sci. Publ. Math. no. 9 (1961), 5-22.

27. P. Orlik and P. Wagreich, Isolated singularities of algebraic surfaces with $C^{*}$ action, Ann. of Math. (2) 93 (1971), 205-228.

28. Singularities of algebraic surfaces with $C^{*}$ action, Math. Ann. 193 (1971), 121-135.

29. O. Riemenschneider, Uber die Anwendung algebraischer Methoden in der Deformations-theorie komplexer Räume, Math. Ann. 187 (1970), 40-55.

30. K. Saito, Einfach-elliptische Singularitäten, Invent. Math. 23 (1974), 289-325.

31. J. P. Serre, Groupes algébriques et corps de classes, Actualités Sci. Indust. no. 1264, Hermann, Paris, 1959.

32. __ Sur les modules projectifs, Sém. Dubrieil 1960/61.

33. Y.-T. Siu, Analytic sheaf cohomology groups of dimension $\boldsymbol{n}$ of $\boldsymbol{n}$-dimensional complex spaces, Trans. Amer. Math. Soc. 143 (1969), 77-94.

34. G. Tyurina, Absolutely isolatedness of rational singularities and triple rational points, Functional Anal. Appl. 2 (1968), 324-332.

35. Ser. Mat. 32 (1968), 943-970. (Russian)

36. __ On a type of contractable curves, Dokl. Akad. Nauk SSSR 173 (1967), 529-531.

37. P. Wagreich, Elliptic singularities of surfaces, Amer. J. Math. 92 (1970), 419-454.

38. Singularities of complex surfaces with solvable local fundamental group, Topology 11 (1972), 51-72. 
39. J. Wahl, Equisingular deformations of plane algebroid curves, Trans. Amer. Math. Soc. 193 (1974), 143-170.

40. O. Zariski, A simplified proof of resolution of singularities of algebraic surfaces, Ann. of Math. (2) 43 (1942), 583-593.

41. __ Studies in equisingularity. I: Equivalent singularities of plane algebroid curves, Amer. J. Math. 87 (1965), 507-536.

42. __ Algebraic surfaces, Springer, Berlin, 1935.

School of Mathematics, Institute for Advanced Study, Princeton, New Jersey 08540

Current address: Department of Mathematics, Harvard University, Science Center, One Oxford Street, Cambridge, Massachusetts 02138 